DESY 02-154

KA-TP-13-2002

PSI-PR-02-10

UB-HET-02-04

\title{
RACOONWW1.3: A Monte Carlo program for four-fermion production at $\mathrm{e}^{+} \mathrm{e}^{-}$colliders
}

\author{
A. Denner ${ }^{\mathrm{a}, 1}$, S. Dittmaier ${ }^{\mathrm{b}}$, M. Roth ${ }^{\mathrm{c}}$, D. Wackeroth ${ }^{\mathrm{d}}$ \\ a Paul Scherrer Institut, CH-5232 Villigen PSI, Switzerland \\ ${ }^{\mathrm{b}}$ Deutsches Electronen-Synchroton DESY, D-22603 Hamburg, Germany \\ ${ }^{\mathrm{c}}$ Institut für Theoretische Physik, Universität Karlsruhe, D-76128 Karslruhe, \\ Germany \\ ${ }^{\mathrm{d} D e p a r t m e n t}$ of Physics, SUNY at Buffalo, Buffalo, NY 14260, USA
}

\begin{abstract}
We present the Monte Carlo generator RACOONWW that computes cross sections to all processes $\mathrm{e}^{+} \mathrm{e}^{-} \rightarrow 4 f$ and $\mathrm{e}^{+} \mathrm{e}^{-} \rightarrow 4 f \gamma$ and calculates the complete $\mathcal{O}(\alpha)$ electroweak radiative corrections to $\mathrm{e}^{+} \mathrm{e}^{-} \rightarrow \mathrm{WW} \rightarrow 4 f$ in the electroweak Standard Model in double-pole approximation. The calculation of the tree-level processes $\mathrm{e}^{+} \mathrm{e}^{-} \rightarrow 4 f$ and $\mathrm{e}^{+} \mathrm{e}^{-} \rightarrow 4 f \gamma$ is based on the full matrix elements for massless (polarized) fermions. When calculating radiative corrections to $\mathrm{e}^{+} \mathrm{e}^{-} \rightarrow \mathrm{WW} \rightarrow 4 f$, the complete virtual doubly-resonant electroweak corrections are included, i.e. the factorizable and non-factorizable virtual corrections in double-pole approximation, and the real corrections are based on the full matrix elements for $\mathrm{e}^{+} \mathrm{e}^{-} \rightarrow 4 f \gamma$. The matching of soft and collinear singularities between virtual and real corrections is done alternatively in two different ways, namely by using a subtraction method or by applying phase-space slicing. Higher-order initial-state photon radiation and naive QCD corrections are taken into account. RACOONWW also provides anomalous triple gauge-boson couplings for all processes $\mathrm{e}^{+} \mathrm{e}^{-} \rightarrow 4 f$ and anomalous quartic gauge-boson couplings for all processes $\mathrm{e}^{+} \mathrm{e}^{-} \rightarrow 4 f \gamma$.
\end{abstract}

Key words: radiative corrections, W-pair production, four-fermion production, triple gauge-boson couplings, quartic gauge-boson couplings, Monte Carlo integration, event generation

PACS: $12.15 \mathrm{Lk}, 12.15 \mathrm{Ji}, 14.70 \mathrm{Fm}$

1 Corresponding author

E-mail: Ansgar.Denner@psi.ch (A. Denner) 


\section{PROGRAM SUMMARY}

Title of program: RACOONWW, version 1.3

Program obtainable from: CPC Program Library, Queen's University of Belfast, N. Ireland

Computer: any computer with FORTRAN 77 compiler. The program has been tested on DEC/ALPHA workstations and Linux PCs.

Operating systems: UNIX, Linux

Programming language used: FORTRAN 77

Memory required to execute with typical data: about $4 \mathrm{MB}$

No. of bytes in distributed program, including test data, etc.: about $2 \mathrm{MB}$

Distribution format: uuencoded compressed tar file

Keywords: radiative corrections, W-pair production, four-fermion production, triple gaugeboson couplings, quartic gauge-boson couplings, Monte Carlo integration, event generation

Nature of physical problem

Precision calculations for W-pair production, four-fermion production, and four-fermion plus photon production in $\mathrm{e}^{+} \mathrm{e}^{-}$annihilation.

\section{Method of solution}

Multi-channel Monte Carlo integration of perturbatively calculated multidifferential cross sections including radiative corrections to $\mathrm{e}^{+} \mathrm{e}^{-} \rightarrow 4 f$. The code can generate weighted and unweighted events. For hadronization an interface to PYTHIA is provided.

Restrictions on the complexity of the problem

Virtual $\mathcal{O}(\alpha)$ radiative corrections are included in double-pole approximation (DPA) only. Higher-order initial-state radiation is included via collinear structure functions in leadinglogarithmic approximation. QCD corrections are included as a global multiplicative factor $\left(1+\alpha_{s} / \pi\right)$ for each hadronically decaying $\mathrm{W}$ boson. The external fermions are assumed to be massless. Below about $170 \mathrm{GeV}$ the DPA is not applicable, and above $500 \mathrm{GeV}$ contributions become numerically important that are not yet implemented in RACOONWW.

Typical running time

On a DEC/ALPHA workstation the computing time for calculating $10^{7}$ weighted events for tree-level predictions is about 2 hours. When including radiative corrections the computing time for $5 \times 10^{7}$ weighted events amounts to about 30 hours. 


\section{LONG WRITE-UP}

\section{Introduction}

Experimental investigations of four-fermion production processes, $\mathrm{e}^{+} \mathrm{e}^{-} \rightarrow 4 f(\gamma)$, are phenomenologically very interesting for a variety of reasons. The most prominent subprocess proceeds via $\mathrm{e}^{+} \mathrm{e}^{-} \rightarrow \mathrm{WW} \rightarrow 4 f$, which is analyzed in the LEP2 experiment at the per-cent level and can be studied with even higher precision at future $\mathrm{e}^{+} \mathrm{e}^{-}$linear colliders, where centre-of-mass (CM) energies go far beyond the $210 \mathrm{GeV}$ reached by LEP2, most probably up to the $\mathrm{TeV}$ range. Upon reconstructing the $\mathrm{W}$ bosons from their decay products, the W-boson mass is determined at LEP2 with an accuracy of presently $\sim 40 \mathrm{MeV}$, close to the finally aimed precision of $\sim 30 \mathrm{MeV}$. Moreover, the triple gauge-boson couplings ZWW and $\gamma \mathrm{WW}$ are directly constrained within a few per cent upon analyzing angular distributions. Recently the LEP2 collaborations started to put bounds on specific deviations from the Standard-Model (SM) quartic gauge-boson couplings, mainly by investigating cross sections and photon-energy distributions in the processes $\mathrm{e}^{+} \mathrm{e}^{-} \rightarrow \mathrm{WW}(\gamma) \rightarrow 4 f \gamma$. Of course, also other subchannels in four-fermion production, such as Z-pair or single-W production, deliver important information that is complementary to results obtained from $\mathrm{W}$ pairs, although the corresponding accuracy at LEP2 is significantly lower. Details about the present situation of the LEP2 data analysis can be found in Ref. [1] and about future prospects at linear colliders in Ref. $[2,3]$.

To account for the high experimental accuracy on the theoretical side is a great challenge: the $\mathrm{W}$ bosons have to be treated as resonances in the full four-fermion processes $\mathrm{e}^{+} \mathrm{e}^{-} \rightarrow 4 f(\gamma)$, and radiative corrections need to be included. From the variety of the above-mentioned physical issues it is also clear that semianalytical predictions are not sufficient; the data analyses require realistic studies with event generators that meet these theoretical requirements. In this article we describe the structure and the use of the Monte Carlo (MC) event generator RACOONWW, which is designed for this task. Numerical results of RACOONWW as well as the underlying calculation of the cross sections and corrections have already been published in Refs. $[4,5,6,7,8]$. A general review of the state-of-the-art for four-fermion production can be found in Refs. [9] and [10]. The latter reference, in particular, contains detailed tuned comparisons of RACOONWW results to related calculations, such as the semi-analytical calculation of Ref. [11] and the MC generator YFSWW3 [12] for $\mathrm{e}^{+} \mathrm{e}^{-} \rightarrow \mathrm{WW} \rightarrow 4 f$ including $\varnothing(\alpha)$ corrections in double-pole approximation (DPA), and the MC generators WRAP [13] and PHEGAS [14] for tree-level predictions of $\mathrm{e}^{+} \mathrm{e}^{-} \rightarrow 4 f \gamma$.

RACOONWW operates in three different modes: the tree-level mode, the improved Born approximation (IBA) mode, or the radiative-correction $(R C)$ mode. The tree-level mode provides predictions for $\mathrm{e}^{+} \mathrm{e}^{-} \rightarrow 4 f$ or $\mathrm{e}^{+} \mathrm{e}^{-} \rightarrow 4 f \gamma$ for all possible final states with massless fermions in lowest order, based on the full set of Feynman diagrams or on selected subsets [4]. Gauge-boson decay widths are implemented as constant or running widths, or within the complex-mass scheme. Since the latter fully respects gauge invariance, gauge- 
invariance violations in the other schemes can be controlled. The initial-state radiation (ISR) in leading-logarithmic (LL) approximation via the structure-function method and the Coulomb singularity for slowly moving $\mathrm{W}$ bosons can be optionally included $[7,8]$. Anomalous triple gauge-boson couplings are supported for all processes $\mathrm{e}^{+} \mathrm{e}^{-} \rightarrow 4 f[15]$, and for the process class $\mathrm{e}^{+} \mathrm{e}^{-} \rightarrow 4 f \gamma$ anomalous quartic gauge-boson couplings are also available $[8]$.

The IBA mode calculates the leading universal corrections to the $\mathrm{e}^{+} \mathrm{e}^{-} \rightarrow \mathrm{WW} \rightarrow 4 f$ processes [7] or to the process class $\mathrm{e}^{+} \mathrm{e}^{-} \rightarrow 4 f \gamma$ [8]. The IBA for $\mathrm{e}^{+} \mathrm{e}^{-} \rightarrow \mathrm{WW} \rightarrow 4 f$, which is similar to the approach followed in the semi-analytical program GENTLE [16], is particularly interesting near the $\mathrm{W}$-pair production threshold where it is still applicable in contrast to the full one-loop calculation in DPA.

The RC mode, which is designed for $\mathrm{e}^{+} \mathrm{e}^{-} \rightarrow 4 f(\gamma)$, uses the $4 f$ lowest-order contribution of the tree-level mode and further includes the full electroweak $\mathcal{O}(\alpha)$ corrections in the DPA as defined in Ref. [6]. More precisely, only the virtual corrections are treated in the DPA leading to factorizable and non-factorizable corrections [17]. The former comprise the corrections to the production and decay subprocesses, while the latter are due to soft-photon exchange between these subprocesses. The real $\mathcal{O}(\alpha)$ corrections are based on the smallest gauge-invariant subset of the full $\mathrm{e}^{+} \mathrm{e}^{-} \rightarrow 4 f \gamma$ matrix element that includes $\mathrm{W}$-pair production. They are evaluated analogously to the tree-level mode for $4 f \gamma$ production. Since the full calculation is consistently based on massless external fermions, photons that are collinear to any external charged fermion have to be recombined with this fermion, as described in Ref. [6] in more detail. RACOONWW provides such recombination algorithms, which can, however, be replaced by user-defined alternatives. Finally, higher-order ISR at the LL level is included up to $\varnothing\left(\alpha^{3}\right)$ using the structure-function approach [18]. In this context, it should be emphasized that the combination of the virtual corrections in DPA with the real corrections based on full matrix elements is non-trivial. The singularity structures of both parts are closely related, and a mismatch has to be carefully avoided. RACOONWW provides two versions for the redistribution of the soft and collinear singularities, resulting in two different branches, called slicing and subtraction branches. The former employs the well-known phase-space slicing, the latter the subtraction procedures of Refs. $[19,20]$. Since even the phase-space generation in the two branches is different, a comparison of results obtained with the different branches is a highly non-trivial consistency check.

Finally, RACOONWW offers various further options, such as the choice between different input-parameter schemes, the inclusion of naive QCD corrections, a switch for the imaginary parts of loop diagrams, and various settings to estimate the intrinsic uncertainty of the DPA. RACOONWW is able to generate unweighted events by a hit-and-miss algorithm. In the RC mode unweighted events can only be generated when using the slicing branch. In order to account for the complex peaking behaviour of the matrix elements RACOONWW uses a multi-channel integration technique [21] and adaptive weight optimization [22].

RACOONWW has been tested up to a CM energy of $\sqrt{s}=500 \mathrm{GeV}$ in the RC mode. 
The theoretical uncertainty of the total cross section has been estimated to be $0.4 \%$ for energies between $200 \mathrm{GeV}$ and $500 \mathrm{GeV}, 0.5 \%$ at $180 \mathrm{GeV}$, and $0.7 \%$ for $170 \mathrm{GeV}$ $[6,10]$. The estimates result from investigations of intrinsic ambiguities in the definition of the DPA and from a detailed comparison with results obtained from the MC generator YFSWW3 [12]. In the RC mode of RACOONWW results have also been obtained for a $\mathrm{CM}$ energy of $1 \mathrm{TeV}$, but no detailed study of the theoretical uncertainties has been performed. Around the W-pair production threshold at $160 \mathrm{GeV}$ the DPA is not applicable, and above $500 \mathrm{GeV}$ higher-order radiative corrections that are not yet implemented in RACOONWW, e.g. large logarithmic electroweak corrections beyond $\varnothing(\alpha)$, may become numerically sizeable. In the tree-level mode RACOONWW is tested up to CM energies of $10 \mathrm{TeV}$ [4], and IBA results have been obtained for CM energies up to $1 \mathrm{TeV}$ [7]. The tree-level and IBA modes are also applicable in the $\mathrm{W}$-pair production threshold region.

The paper is organized as follows: in Section 2, we sketch the general structure of the program. Section 3 is devoted to the description of important details of the code. In Section 4, we describe in detail the usage of RACOONWW including all important options. Section 5 summarizes the paper, and the appendix contains input and output of two sample runs.

\section{Program structure}

RACOONWW provides predictions for the cross section to the class of processes $\mathrm{e}^{+} \mathrm{e}^{-} \rightarrow$ $4 f(\gamma)$ as given by

$$
\int \mathrm{d} \sigma=\frac{(2 \pi)^{4-3 n}}{2 s} \int \mathrm{d} \Phi^{(n)}\left|\mathcal{M}\left(p_{+}, p_{-}, k_{3}, \ldots, k_{n+2}\right)\right|^{2},
$$

where $p_{ \pm}$and $k_{i}$ are the momenta of the incoming and outgoing particles, respectively, and $n=4,5$ is the number of outgoing particles. The total energy of the scattering process

in the CM frame, where $\mathbf{p}_{+}+\mathbf{p}_{-}=\mathbf{0}$, is denoted by $\sqrt{s}=p_{+}^{0}+p_{-}^{0}$. The $n$-particle phase space is defined by

$$
\mathrm{d} \Phi^{(n)}=\left.\prod_{i=3}^{n+2} \frac{\mathrm{d}^{3} \mathbf{k}_{i}}{2 k_{i}^{0}} \delta^{(4)}\left(p_{+}+p_{-}-\sum_{i=3}^{n+2} k_{i}\right)\right|_{k_{i}^{0}=\sqrt{\mathbf{k}_{i}^{2}+m_{i}^{2}}} .
$$

Results for the matrix element squared $|\mathcal{M}|^{2}$ in terms of helicity amplitudes for the treelevel processes $\mathrm{e}^{+} \mathrm{e}^{-} \rightarrow 4 f(\gamma)$ with massless fermions are given in Ref. [4], and the calculation of radiative corrections is described in Ref. [6]. The phase-space integration is performed with the help of a $\mathrm{MC}$ technique, which allows to calculate a variety of observables simultaneously and to easily implement separation cuts and photon recombination schemes in order to account for the experimental situation. The matrix element squared exhibits a complicated peaking behaviour in various regions of the integration domain. In order to obtain a numerically stable result and to reduce the $\mathrm{MC}$ integration error, 
RACOONWW is based on a multi-channel MC method [21] with adaptive weight optimization [22].

The general structure of the program is determined by the generation of the $n$-particle phase space, $\Phi^{(n)}$, and the evaluation of the matrix element squared, $|\mathcal{M}|^{2}$. The program run starts with some initializations and ends with the output of the integration result in form of an output file for the total cross section and a number of data files for various distributions.

The initialization of the matrix-element evaluation consists to a large extent of specifying the input parameters and the different options provided by RACOONWW. The initialization of the phase-space generation in the multi-channel MC integration approach essentially amounts to the identification of kinematical channels for the given final state. While the user is free to choose the parameters and flags for options in the input file (and to a lesser extent also in racoonww.f and public.f), the initialization of the multi-channel phase-space generators is done automatically by RACOONWW.

RACOONWW consists of 11 fortran files and a makefile. The usage of the program is described in the file README. RACOONWW also includes 5 default input files with corresponding output and data files. They represent the recommended choices for input parameters and flags of the different modes provided by RACOONWW. The general structure of the program is reflected by a series of subroutine calls in the main program in racoonww. $\mathrm{f}$ :

\section{CALL READINPUT : the input file is read. \\ CALL INITIALIZE : parameters and flags are set, and the MC integration is ini- tialized (specification of channels for multi-channel integra- tion). \\ CALL INTEGRATION : the MC integration and the generation of NEVENTSW weighted events are performed, the hit-and-miss algorithm for gener- ation of unweighted events is initialized. \\ CALL EVENTGENERATION : in the main program it is only called if NEVENTSUNW $>0$; then NEVENTSUNW unweighted events are generated by using a hit-and-miss algorithm (see Section 4.4). This subroutine is also called in the subroutine INTEGRATION where it generates weighted events. \\ CALL PY4FRM : the interface to PYTHIA [23] to include parton showering and hadronization is called. The subroutine PY4FRM is provided by Pyтhia (see also Section 4.4). \\ CALL WRITEOUTPUT : the results are written to the output file and data files.}

Besides the choices made for the input parameters and flags in the input file, the user can change the default values for the remaining parameters and flags of RACOONWW in the subroutine INITIALIZE in racoonww. $f$ and in the subroutine PARAMETER in public.f. The specification of the input in RACOONWW is described in detail in Section 4.1. After the initialization, the $\mathrm{MC}$ integration is performed in 
SUBROUTINE INTEGRATION (AVPREL, SIPREL)

AVPREL : total cross section calculated with NEVENTSW weighted events, SIPREL : corresponding statistical error

by calling the MC kernels in

SUBROUTINE EVENTGENERATION (ATOTSQR , A1SQR, A2SQR, UNWEIGHTING)

ATOTSQR : total matrix element squared for the event (input for PY4FRM),

A1SQR : matrix element squared for the configuration with fermions $f_{3}, \bar{f}_{4}$ and $f_{5}, \bar{f}_{6}$ as the two colour singlets (input for PY4FRM),

A2SQR : matrix element squared for the configuration with fermions $f_{3}, \bar{f}_{6}$ and $f_{5}, \bar{f}_{4}$ as the two colour singlets (input for PY4FRM),

UNWEIGHTING : flag for generating weighted (UNWEIGHTING $=0$ ) or unweighted (UNWEIGHTING = 1) events; this flag is automatically controlled by NEVENTSUNW.

RACOONWW provides an unweighting procedure based on a hit-and-miss algorithm. It is activated when the user sets NEVENTSUNW $>0$ in the main program, where NEVENTSUNW is the number of unweighted events. When generating unweighted events, parton showering and hadronization for coloured final states can be taken into account by activating the interface to PYTHIA [23] in the main program. The information about the (unweighted) event is stored in the HEP standard format [24] in the common block HEPEVT (see Section 4.4). The variables ATOTSQR, A1SQR, and A2SQR are provided for the generation of unweighted events. A detailed discussion of the unweighting procedure is given in Section 4.4 .

By default RACOONWW generates only weighted events (NEVENTSUNW $=0$ ). The number of weighted events, NEVENTSW, is set in the input file. The events are represented by the four-momenta of the final-state particles and their corresponding weights. A weight, $w\left(r_{0}^{j}, \mathbf{r}^{j}\right)$, assigned to the MC point $\left(r_{0}^{j}, \mathbf{r}^{j}\right)$ of the $j$ th event, is composed of the total density of the event, $g_{\text {tot }}$, (cf. Section 3.1.2) and the matrix element squared as

$$
w\left(r_{0}^{j}, \mathbf{r}^{j}\right)=\frac{f\left(k_{i}\right)}{g_{\text {tot }}\left(k_{i}\right)} \quad \text { with } \quad f\left(k_{i}\right)=\frac{(2 \pi)^{4-3 n}}{2 s}\left|\mathcal{M}\left(k_{i}\right)\right|^{2},
$$

where $r_{0}^{j}, \mathbf{r}^{j}$ is a set of pseudo-random numbers. As usual, the MC estimate $\bar{I}$ of the total cross section is obtained as $(N \equiv$ NEVENTSW $)$

$$
\bar{I}=\frac{1}{N} \sum_{j=1}^{N} w\left(r_{0}^{j}, \mathbf{r}^{j}\right)
$$

In the RC mode of RACOONWW more than one weight per (weighted) event is generated corresponding to the kinematics of the subcontributions, i.e. whether the weight is a $4 f$ or $4 f \gamma$ event with or without ISR, or corresponding to the DPA part of the virtual 
corrections, or to one of the subtraction terms. The weights for each weighted event (see Tables 5-7) together with the corresponding four-momenta are provided in the common block EVENT in racoonww.f:

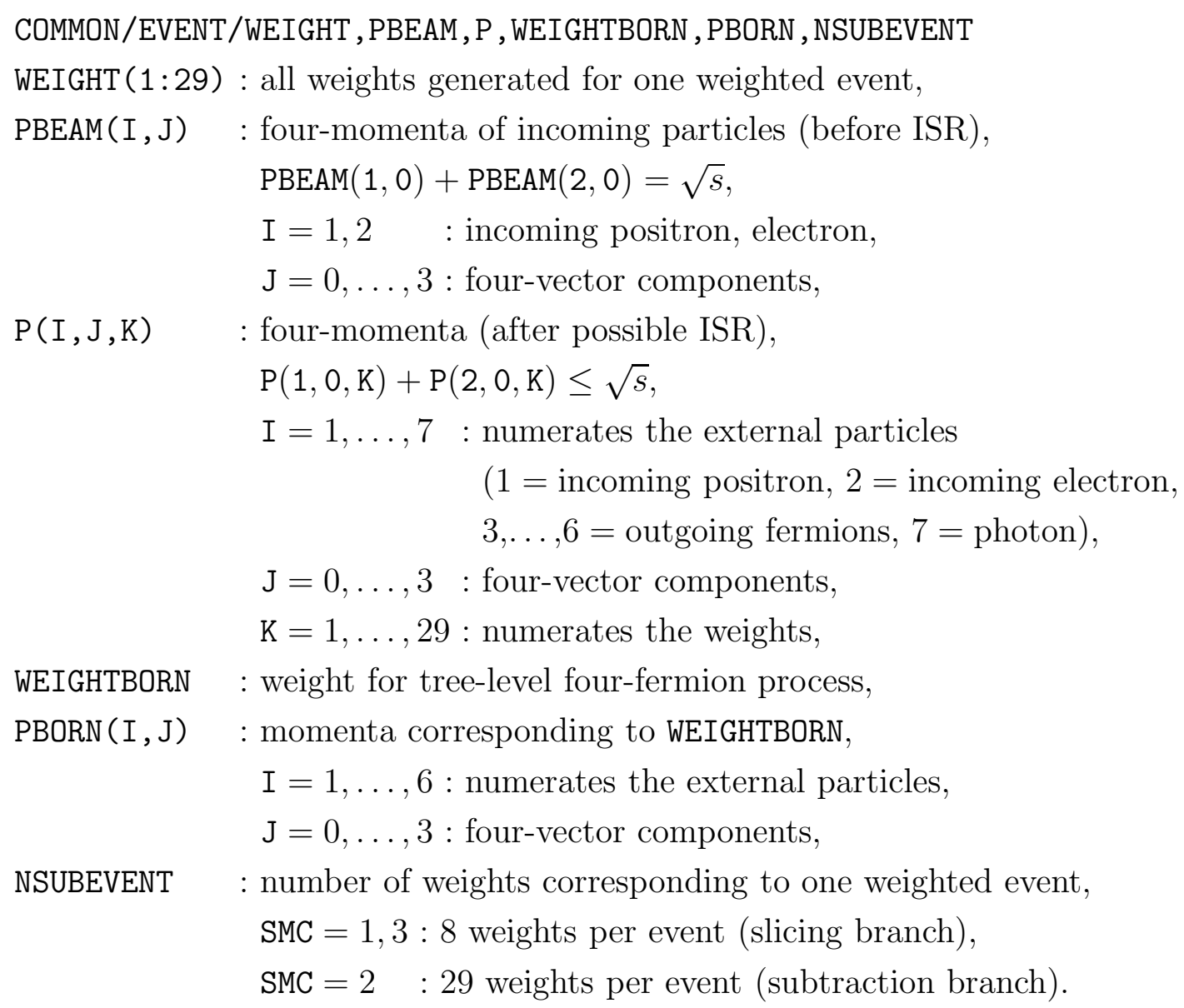

These two key components of the event generation, phase-space generation and matrixelement evaluation, are described in detail in Sections 3.1 and 3.2, respectively.

For a realistic simulation of $\mathrm{e}^{+} \mathrm{e}^{-} \rightarrow 4 f(\gamma)$ processes separation cuts and a photon recombination procedure have to be applied to take into account the detector response. RACOONWW provides several experiment-inspired recombination procedures and sets of separation cuts, which can be specified in the input file. They are defined in public.f in

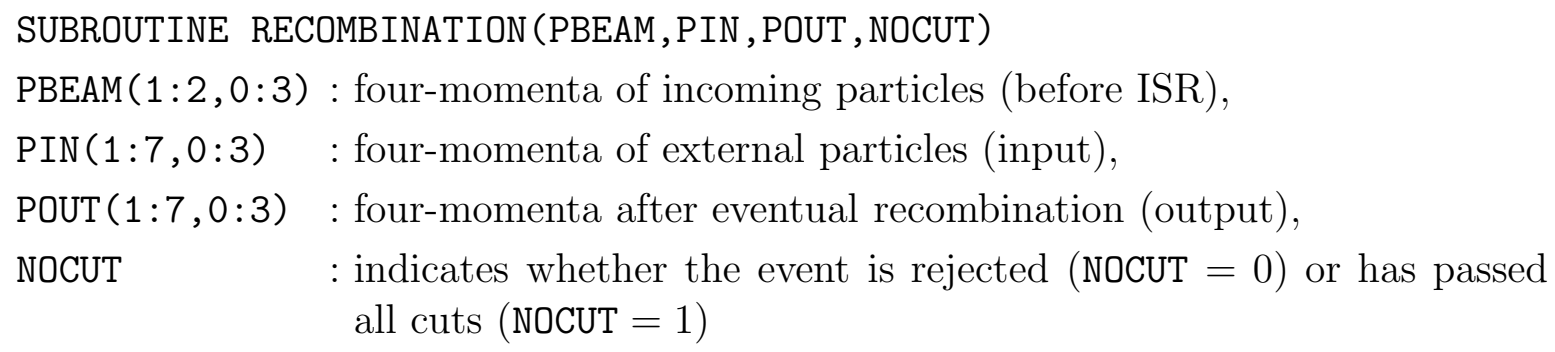

and 
SUBROUTINE CUT (PBEAM, P, NPARTICLE, NOCUT)

$\operatorname{PBEAM}(1: 2,0: 3)$ : four-momenta of incoming particles (before ISR),

$\mathrm{P}(1: 7,0: 3) \quad$ : four-momenta of external particles (after possible ISR),

NPARTICLE : number of particles in the final state,

PBEAM, NOCUT : see subroutine RECOMBINATION.

Finally, the data files for the histograms are filled in subroutine SETTING in public.f, and the results for the total and differential cross sections are written to the output and data files in the subroutine WRITEOUTPUT.

In the following we describe in detail the most important features of the program and its usage.

\section{Details of the program}

RACOONWW is a merger of two MC programs, which use the same routines for the evaluation of the matrix elements but different, completely independent routines for the phase-space generation. Moreover, they differ in the treatment of the soft and collinear singularities arising in the calculation of the radiative corrections to $\mathrm{e}^{+} \mathrm{e}^{-} \rightarrow \mathrm{WW} \rightarrow 4 f$. One employs the subtraction method of Refs. $[19,20]$ and the other applies phase-space slicing (for a review see e.g. Ref. [25]). The two branches of RACOONWW are named accordingly, the subtraction and slicing branches, and the corresponding MC integration kernels are

FUNCTION KERN (XV,WGT)

$\mathrm{XV}(1: 15)$ : pseudo-random numbers for the generation of one weighted event,

WGT : not used in this version of RACOONWW

for the slicing branch in kern.f and

SUBROUTINE SUBTRACTION (RANDOM)

RANDOM (1:15) : pseudo-random numbers for the generation of one weighted event

for the subtraction branch in subtraction.f, respectively. The pseudo-random number generator RANS can be found at the end of racoonww. f. The user chooses between these two branches by setting the flag SMC in the input file, SMC $=1$ or 3 for the slicing branch and $\mathrm{SMC}=2$ for the subtraction branch. The value $\mathrm{SMC}=3$ has to be used when unweighted events are generated in the $\mathrm{RC}$ mode. The initialization of the event generation and the setting of branch-specific options for the slicing and subtraction branches of RACOONWW is done in

SUBROUTINE INITSLICING (ENERGY, CONV , SCALE, NEVENTSW, NOUT , IOUT)

ENERGY : CM energy in GeV $($ ENERGY $=\sqrt{s})$, 
CONV : conversion factor from $\mathrm{GeV}^{-2}$ to fbarn,

SCALE : QED splitting scale in the structure functions (see Ref. [6] and Section 3.2),

NEVENTSW : number of weighted events,

NOUT : number assigned to the output file,

IOUT : number assigned to the file optimization.info

in slicing. $f$ and

SUBROUTINE INITSUBTRACTION (ENERGY, CONV , SCALE , NOUT)

ENERGY, CONV , SCALE, NOUT : see subroutine INITSLICING

in subtraction.f. In particular, the specification of the kinematical channels for the multi-channel MC integration for the chosen four-fermion final state is done automatically in these routines.

After the initialization, the MC kernels are called. The phase-space cuts are applied first, and if the event is not discarded, the total phase-space weight, $1 / g_{\text {tot }}$, and the matrix element squared, $|\mathcal{M}|^{2}$, of Eq. (3) are calculated. For the matrix-element evaluation the user can choose between the tree-level, IBA, and RC modes of RACOONWW by setting the flag SRC in the input file. The implementation of the three modes is described in detail in Section 3.2.

\subsection{The phase-space generation and multi-channel MC integration}

The numerical integration of Eq. (1) is rather complicated owing to the rich peaking structure of the integrand, since the amplitudes to $\mathrm{e}^{+} \mathrm{e}^{-} \rightarrow 4 f$ and, in particular, to $\mathrm{e}^{+} \mathrm{e}^{-} \rightarrow 4 f \gamma$ involve a huge number of propagators that become resonant or are enhanced in various phase-space regions. To obtain reliable numerical results, events have to be sampled more frequently in the integration domains where the integrand is large. This redistribution of events is called importance sampling. In practice, this means that the mapping of the pseudo-random numbers $r_{i}, 0 \leq r_{i} \leq 1$, into the space of final-state momenta has to be chosen such that the corresponding Jacobian $1 / g(\mathbf{r})$ cancels the propagators of the differential cross section at least partially.

\subsubsection{Propagator mappings}

In order to smoothen a single propagator with momentum transfer $q$ in the square of a matrix element,

$$
|\mathcal{M}|^{2} \propto \frac{1}{\left(q^{2}-M^{2}\right)^{2}+M^{2} \Gamma^{2}}
$$


the phase space is parametrized in such a way that the virtuality $q^{2}$ of the propagator is chosen as an integration variable. The integral over $q^{2}$ is transformed into an integral over the random number $r$ :

$$
\int_{q_{\min }^{2}}^{q_{\max }^{2}} \mathrm{~d} q^{2}=\int_{0}^{1} \mathrm{~d} r \frac{1}{g_{\operatorname{prop}}\left(q^{2}(r)\right)} .
$$

The dependence of $q^{2}$ on the pseudo-random number $r$ has to be chosen such that the Jacobian $1 / g_{\text {prop }}$ smoothens the propagator (5). In RACOONWW two types of mappings are used according to the mass $M$ of the propagating particle. Since all occurring massive particles possess a finite decay width $\Gamma$, a suitable mapping is of Breit-Wigner type $(M \neq 0, \Gamma \neq 0)$ :

$$
\begin{aligned}
q^{2}(r) & =M \Gamma \tan \left[y_{1}+\left(y_{2}-y_{1}\right) r\right]+M^{2}, \\
g_{\text {prop }}\left(q^{2}\right) & =\frac{M \Gamma}{\left(y_{2}-y_{1}\right)\left[\left(q^{2}-M^{2}\right)^{2}+M^{2} \Gamma^{2}\right]}
\end{aligned}
$$

with

$$
y_{1,2}=\arctan \left(\frac{q_{\min , \max }^{2}-M^{2}}{M \Gamma}\right) .
$$

For massless particles the following mappings are appropriate $(M=\Gamma=0)$ :

$$
\begin{aligned}
q^{2}(r) & =\left[r\left(q_{\max }^{2}\right)^{1-\nu}+(1-r)\left(q_{\min }^{2}\right)^{1-\nu}\right]^{\frac{1}{1-\nu}} \\
g_{\text {prop }}\left(q^{2}\right) & =\frac{1-\nu}{\left(q^{2}\right)^{\nu}\left[\left(q_{\max }^{2}\right)^{1-\nu}-\left(q_{\min }^{2}\right)^{1-\nu}\right]} \\
q^{2}(r) & =\left(q_{\max }^{2}\right)^{r}\left(q_{\min }^{2}\right)^{1-r}, \quad g_{\text {prop }}\left(q^{2}\right)=\frac{1}{q^{2} \ln \left(q_{\max }^{2} / q_{\min }^{2}\right)} .
\end{aligned}
$$

The mapping of Eq. (9) is only valid for $\nu \neq 1$, while Eq. (10) is a substitute of Eq. (9) for $\nu=1$. The naive expectation $\nu=2$ from the squared matrix element is not necessarily the best choice because the propagator poles are partially cancelled in the collinear limit. It turns out that a proper value is $\nu \lesssim 1$.

In this way all propagators of a given Feynman diagram can be smoothened by choosing an appropriate set of integration variables and mappings as given in Eqs. (7)-(10). The integration variables that do not correspond to a specific propagator mapping are sampled uniformly, i.e.

$$
q^{2}(r)=q_{\max }^{2} r+q_{\min }^{2}(1-r), \quad \phi(r)=2 \pi r, \quad \cos \theta(r)=2 r-1
$$


for invariant masses $q^{2}$, azimuthal angles $\phi$, and polar angles $\theta$, respectively.

\subsubsection{Multi-channel approach}

Obviously, importance sampling of all propagators appearing in the amplitude is not possible by a single phase-space mapping. The multi-channel approach [21] provides a solution to this problem. For each peaking structure a suitable set of invariants is chosen, and accordingly a mapping of random numbers $\mathbf{r}$ into the final-state momenta $k_{i}$, so that the resulting Jacobian of this mapping $1 / g_{k}$ cancels this particular peaking behaviour of $f\left(k_{i}\right)$. The phase-space integral of Eq. (1) reads

$$
\int \mathrm{d} \Phi^{(n)}=\int_{0}^{1} \mathrm{~d} r_{0} \sum_{k=1}^{N_{\mathrm{ch}}} \theta\left(r_{0}-\beta_{k-1}\right) \theta\left(\beta_{k}-r_{0}\right) \int_{0}^{1} \mathrm{~d}^{3 n-4} \mathbf{r} \frac{1}{g_{\mathrm{tot}}\left(k_{i}(\mathbf{r})\right)}
$$

where the $\beta_{i}$ define a partition of unity, i.e. $\beta_{i}=\sum_{j=1}^{i} \alpha_{j}, \beta_{0}=0, \beta_{N_{\mathrm{ch}}}=\sum_{j=1}^{N_{\mathrm{ch}}} \alpha_{j}=1$, and $N_{\text {ch }}$ denotes the number of all channels. In Eq. (12) an additional random number $r_{0}$ is introduced in order to select a channel $k$ randomly with probability $\alpha_{k} \geq 0$. The numbers $\alpha_{k}$ are called a priori weights. All local densities $g_{k}$ are combined into the total density $g_{\text {tot }}$,

$$
g_{\mathrm{tot}}=\sum_{k=1}^{N_{\mathrm{ch}}} \alpha_{k} g_{k}
$$

which is expected to smoothen the integrand over the whole phase space. In this way, it is possible to simultaneously include different phase-space mappings which are suitable for different parts of the integrand. To be specific, for each (tree-level) Feynman diagram one channel exists, so that all propagators (except massive $t$-channel propagators in the slicing branch) of the squared diagram are smoothened by the corresponding local density $g_{k}$. The local densities are obtained as the product of the corresponding Jacobians, as given in Eqs. (7)-(10).

The a priori weights $\alpha_{k}$ are adapted in the early phase of the MC run several times to optimize the convergence behaviour of the numerical integration. This adaptive weight optimization [22] increases the a priori weights $\alpha_{k}$ for such channels that give large contributions to the total cross section. After a certain number of generated events a new set of a priori weights $\alpha_{k}^{\text {new }}$ is calculated according to

$$
\alpha_{k}^{\text {new }} \propto \alpha_{k} \sqrt{\frac{1}{N} \sum_{j=1}^{N} \frac{g_{k}\left(k_{i}\left(\mathbf{r}^{j}\right)\right)\left[w\left(r_{0}^{j}, \mathbf{r}^{j}\right)\right]^{2}}{g_{\text {tot }}\left(k_{i}\left(\mathbf{r}^{j}\right)\right)}}, \quad \sum_{k=1}^{N_{\mathrm{ch}}} \alpha_{k}^{\text {new }}=1 .
$$


The error of the MC integration can be estimated as

$$
\delta \bar{I}=\sqrt{\frac{W(\alpha)-\bar{I}^{2}}{N}}, \quad W(\alpha)=\frac{1}{N} \sum_{j=1}^{N}\left[w\left(r_{0}^{j}, \mathbf{r}^{j}\right)\right]^{2}
$$

with the MC estimate $\bar{I}$ of Eq. (4).

The phase-space integration is implemented in RACOONWW as follows:

- First the phase-space generators are initialized by identifying all channels needed to account for the peaking behaviour of the process under consideration. For the processes $\mathrm{e}^{+} \mathrm{e}^{-} \rightarrow 4 f$, there are between 6 and 128 different channels, for $\mathrm{e}^{+} \mathrm{e}^{-} \rightarrow 4 f \gamma$ between 14 and 928 channels. Each channel smoothens a particular combination of propagators that results from a characteristic Feynman diagram. The identification of the channels in the slicing and subtraction branches of RACOONWW is described in Section 3.1.4 and Section 3.1.5, respectively.

- The channel that is used to generate the event is picked randomly with probability $\alpha_{k}$. Using the randomly chosen channel, the momentum configuration $k_{i}$ is determined from the corresponding phase-space generator. Finally, using the momentum configuration $k_{i}$ the local densities of all channels $g_{k}$ are calculated. From those densities the total density $g_{\text {tot }}$ of the event is determined. Apart from the evaluation of the matrix elements, the calculation of the local densities is the most time-consuming part of the numerical integration.

- In order to reduce the $\mathrm{MC}$ error further, the a priori weights $\alpha_{k}$ are recalculated several times in the MC integration according to Eq. (14).

\subsubsection{The phase-space generators}

The actual calculation of the event kinematics is decomposed into three steps: the calculation of time-like invariants $s_{i}$, of $2 \rightarrow 2$ scattering processes, and of $1 \rightarrow 2$ decays. The phase-space integration of Eq. (1) reads

$$
\int \mathrm{d} \Phi^{(n)}=\prod_{i=1}^{n-2} \int_{s_{i, \min }}^{s_{i, \max }} \mathrm{d} s_{i} \prod_{j=1}^{\tau} \int \mathrm{d} \Phi_{j}^{2 \rightarrow 2} \prod_{k=1}^{n-1-\tau} \int \mathrm{d} \Phi_{k}^{1 \rightarrow 2}
$$

where the phase spaces of the $2 \rightarrow 2$ scattering processes and the $1 \rightarrow 2$ decays are denoted by $\Phi_{j}^{2 \rightarrow 2}$ and $\Phi_{k}^{1 \rightarrow 2}$, respectively. The $n-2$ invariants $s_{i}$ result from the phasespace factorization into scattering processes and decays. The number $\tau$ of $2 \rightarrow 2$ scattering processes can range from 1 to $n-1$. If there is only one $2 \rightarrow 2$ scattering process $(\tau=1)$, this can involve either a $t$-channel or an $s$-channel propagator. If there are more scattering processes $(\tau \geq 2)$, these involve only $t$-channel propagators. If only one $t$-channel $2 \rightarrow 2$ scattering process is present, i.e. for the first two topologies in the first row of Figure 1 and the first three topologies in the second row of Figure 1, all the $s_{i}$ correspond to the 


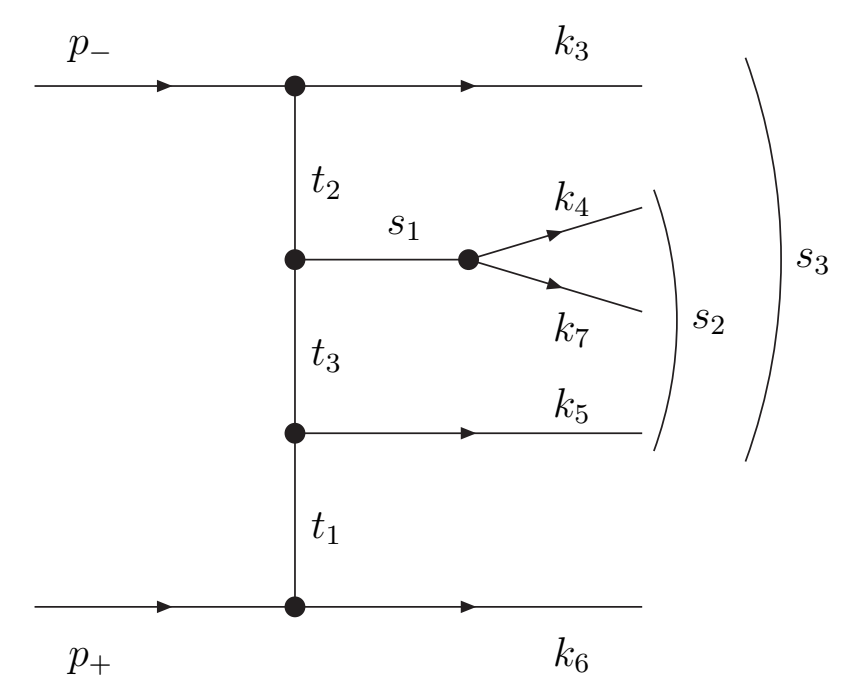

Fig. 1. Generic diagram of a specific channel

virtualities of propagators and are mapped as described in Section 3.1.1. The angles and invariants appearing in the parametrizations of the phase spaces are sampled as described below. If several $t$-channel propagators are present, i.e. for $\tau \geq 2$, some of the $s_{i}$ do not correspond to virtualities of propagators in the diagram; for such variables no mappings are introduced, they are generated uniformly. Topologies with no $t$-channel propagator, are obtained from those with only one $t$-channel propagator by replacing the $t$-channel scattering process with an $s$-channel scattering process. The corresponding invariant is fixed to the CM energy squared $s$, and the phase space is parametrized as for the $1 \rightarrow 2$ decays in Eq. (20) or equivalently for the $2 \rightarrow 2$ scattering processes in Eq. (18) without propagator mapping $(\nu=M=\Gamma=0)$. Diagrams with four-particle vertices result from the contraction of propagators in the diagrams of Figure 1. Consequently, they also fit into the decomposition (16), and the invariants corresponding to the contracted propagators are sampled uniformly. More details on phase-space parametrizations can be found in Refs. [20,26].

For each class of diagrams with the same propagator structure we adopt an own channel for the numerical integration. No special channels are provided for interference contributions.

We illustrate the general strategy by considering the specific topology shown in Figure 1, where a particular choice for the incoming and outgoing momenta $p_{ \pm}$and $k_{i}$ is made. For this example the phase-space parametrization Eq. (16) reads

$$
\begin{aligned}
\left.\int \mathrm{d} \Phi^{(n)}\right|_{\text {Fig. } 1}= & \prod_{i=1}^{3} \int_{s_{i, \text { min }}}^{s_{i, \max }} \mathrm{d} s_{i} \int \mathrm{d} \Phi^{2 \rightarrow 2}\left(p_{+}, p_{-} ; k_{6}, k_{3457}\right) \int \mathrm{d} \Phi^{2 \rightarrow 2}\left(p_{-}, p_{+}-k_{6} ; k_{3}, k_{457}\right) \\
& \times \int \mathrm{d} \Phi^{2 \rightarrow 2}\left(p_{+}-k_{6}, p_{-}-k_{3} ; k_{5}, k_{47}\right) \int \mathrm{d} \Phi^{1 \rightarrow 2}\left(k_{4}, k_{7}\right)
\end{aligned}
$$

where sums of outgoing momenta are abbreviated by $k_{i j}=k_{i}+k_{j}, k_{i j k}=k_{i}+k_{j}+k_{k}$, 
etc. Note that only the time-like invariant $s_{1}=k_{47}^{2}$ corresponds to a virtuality of a propagator in the diagram, while $s_{2}=k_{457}^{2}$ and $s_{3}=k_{3457}^{2}$ correspond to invariant masses of fictitious final-state particles within the first two $2 \rightarrow 2$ scattering processes, symbolized by the arches in Figure 1. More examples for the phase-space generation can be found in Refs. [4,20].

In detail we proceed as follows.

\section{Calculation of time-like invariants}

First of all, the time-like invariants $s_{i}$ are determined. The invariants corresponding to $s$-channel propagators are calculated from Eqs. (7)-(10). All other time-like invariants are sampled uniformly, i.e. calculated from Eq. (11). The determination of the invariants $s_{i}$ is ordered in such a way that the $s_{i}$ nearest to final-state particles are calculated first, followed by the next-to-nearest $s$-channel propagators, and so on. The lower and upper limits $s_{i, \min / \max }$, in general, are functions of the already determined invariants. To enhance the efficiency of the numerical integration in the presence of separation cuts, in some cases these cuts are taken into account in the evaluation of $s_{i, \min / \max }$.

\section{$2 \rightarrow 2$ scattering processes}

The phase spaces for the $2 \rightarrow 2$ scattering processes of Eq. (16) are parametrized as

$$
\int \mathrm{d} \Phi^{2 \rightarrow 2}\left(q_{1}, q_{2} ; p_{1}, p_{2}\right)=\frac{1}{8 \sqrt{\left(q_{1} q_{2}\right)^{2}-q_{1}^{2} q_{2}^{2}}} \int_{0}^{2 \pi} \mathrm{d} \phi^{*} \int_{t_{\min }}^{t_{\max }} \mathrm{d} t
$$

where $q_{1,2}$ are the incoming and $p_{1,2}$ are the outgoing momenta, and $\phi^{*}$ is the azimuthal angle defined by $p_{1}$ and $q_{1}$ in the CM frame of the subprocess where $\mathbf{q}_{1}+\mathbf{q}_{2}=0$. The argument $t=\left(p_{1}-q_{1}\right)^{2}$ of the $t$-channel propagator is calculated according to Eqs. (7)-(10) for $t$-channel scattering processes (apart from massive $t$-channel propagators in the slicing branch that are sampled uniformly) and according to Eq. (11) for $s$-channel scattering processes. The azimuthal angle $\phi^{*}$ is sampled uniformly. Since the corresponding polar angle $\cos \theta^{*}$ of this scattering process depends on the invariant $t$ linearly,

$$
t=\frac{q_{1}^{2} p_{2}^{2}+q_{2}^{2} p_{1}^{2}-2\left(q_{1} q_{2}\right)\left(p_{1} p_{2}\right)+2 \sqrt{\left(q_{1} q_{2}\right)^{2}-q_{1}^{2} q_{2}^{2}} \sqrt{\left(p_{1} p_{2}\right)^{2}-p_{1}^{2} p_{2}^{2}} \cos \theta^{*}}{\left(q_{1}+q_{2}\right)^{2}}
$$

the calculation of the momenta and the Lorentz transformation into the laboratory (LAB) frame is simple and, thus, skipped here. Explicit formulas can, for instance, be found in Refs. [20,26].

Cuts on angles between outgoing particles and the beams effectively reduce the integration range of $t$. For the $2 \rightarrow 2$ scattering processes that are attached to the original incoming 


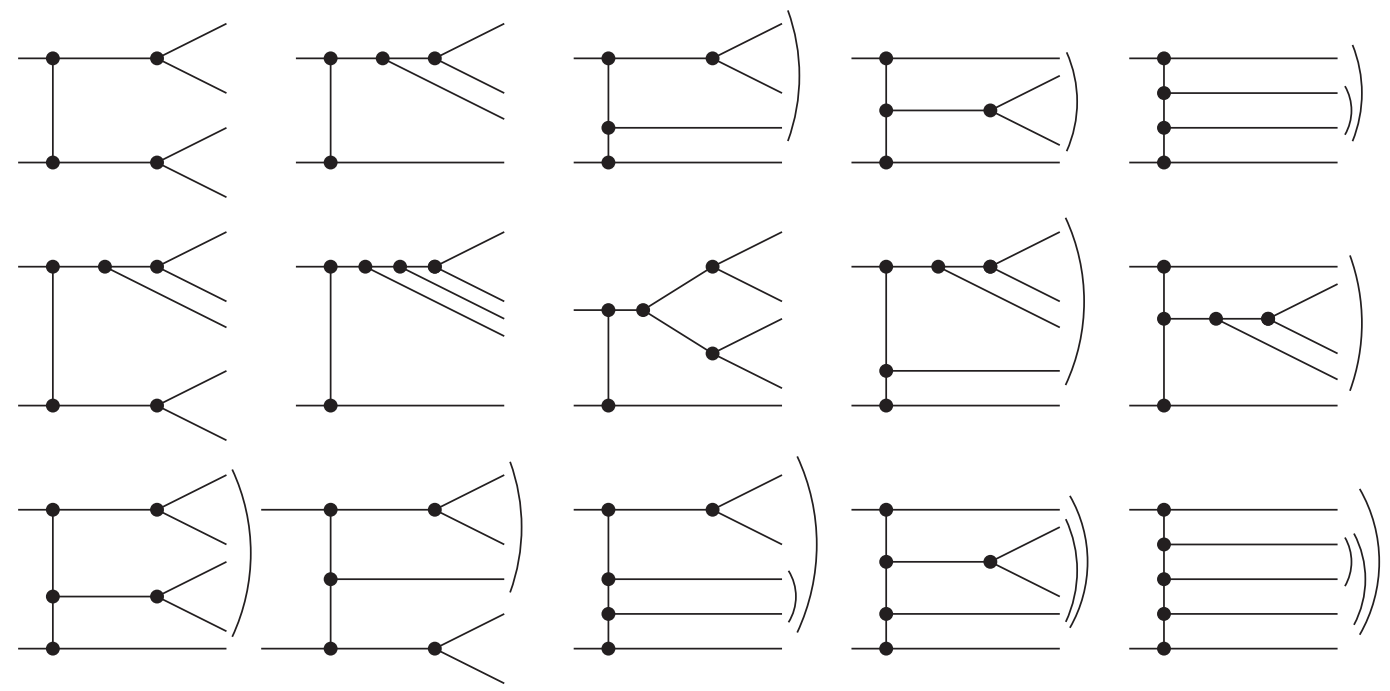

Fig. 2. The generic phase-space generators symbolized by the topologies of the corresponding diagrams. The arcs indicate the intermediate $2 \rightarrow 2$ scattering processes used in the phase-space parametrizations. Topologies with an $s$-channel $2 \rightarrow 2$ scattering process are obtained from the ones with only one $t$-channel scattering process by contracting the $t$-channel propagator.

particles these cuts are in some cases included in the determination of $t_{\min / \max }$ to enhance the efficiency.

\section{$1 \rightarrow 2$ particle decays}

The phase-space integration for the $1 \rightarrow 2$ particle decays in Eq. (16) reads

$$
\int \mathrm{d} \Phi^{1 \rightarrow 2}\left(p_{1}, p_{2}\right)=\frac{\sqrt{\left(p_{1} p_{2}\right)^{2}-p_{1}^{2} p_{2}^{2}}}{4\left(p_{1}+p_{2}\right)^{2}} \int_{0}^{2 \pi} \mathrm{d} \phi^{*} \int_{-1}^{1} \mathrm{~d} \cos \theta^{*}
$$

where $\phi^{*}$ and $\theta^{*}$ are the azimuthal and polar angles in the rest frame of the decaying particle, respectively. The variables $\phi^{*}$ and $\cos \theta^{*}$ are sampled uniformly. The momenta of the outgoing particles are $p_{1}$ and $p_{2}$. As in the former step, the calculation of the momenta and the Lorentz transformation into the LAB frame is straightforward.

All topologies corresponding to the generic phase-space generators for the processes $\mathrm{e}^{+} \mathrm{e}^{-} \rightarrow 4 f$ and $\mathrm{e}^{+} \mathrm{e}^{-} \rightarrow 4 f \gamma$ are shown in Figure 2 .

\subsubsection{Phase-space generation in the slicing branch}

In the slicing branch, the $2 \rightarrow 2$ scattering processes and the $1 \rightarrow 2$ decays are combined in the function

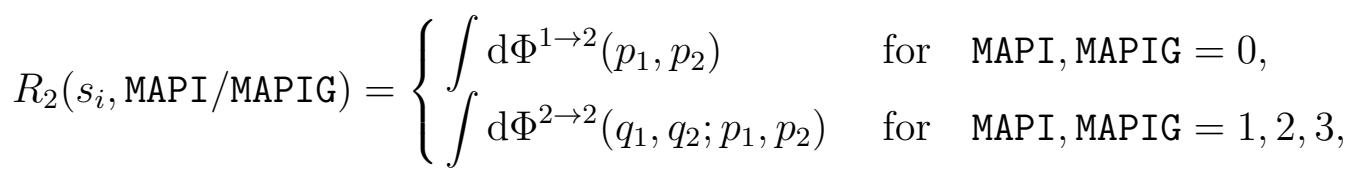


$\operatorname{MAPI}(6)=1:$

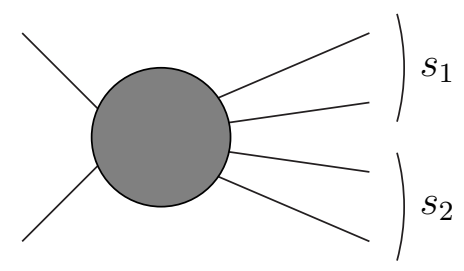

$\operatorname{MAPI}(6)=2$ :

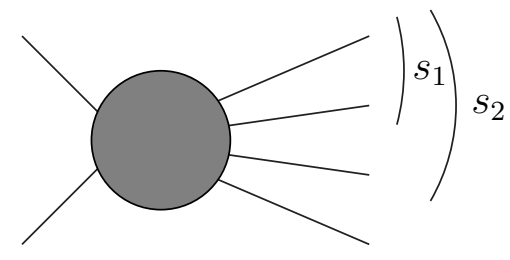

$\operatorname{MAPIG}(6)=1: \quad \operatorname{MAPIG}(6)=2: \quad \operatorname{MAPIG}(6)=3:$

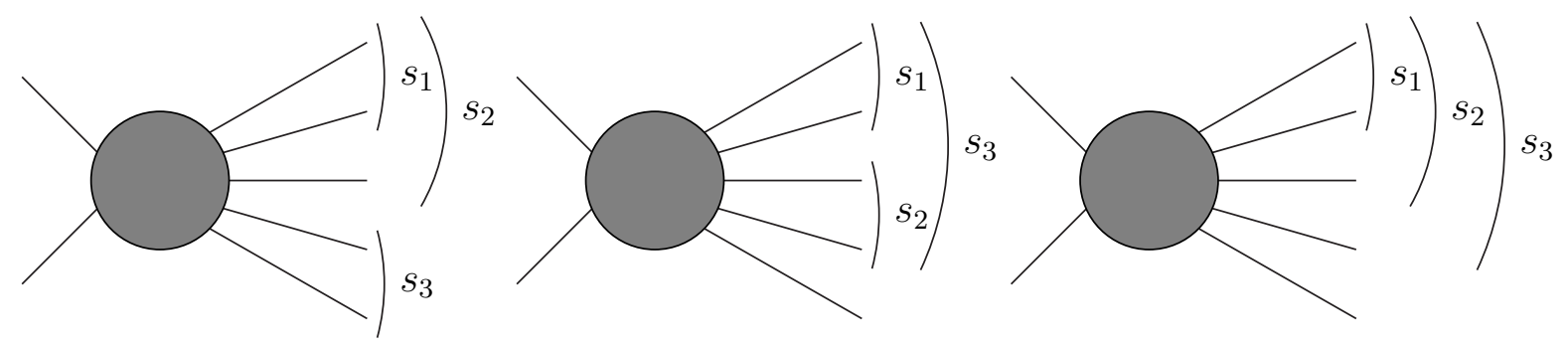

Fig. 3. The basic phase-space decompositions from which all channels for the processes $\mathrm{e}^{+} \mathrm{e}^{-} \rightarrow 4 f$ and $\mathrm{e}^{+} \mathrm{e}^{-} \rightarrow 4 f \gamma$ can be constructed, as used in the slicing branch

where $s_{i}=\left(p_{1}+p_{2}\right)^{2}$. Using the function $R_{2}$, the phase-space integration of Eq. (16) reads

$$
\begin{aligned}
\int \mathrm{d} \Phi^{(4)}= & \int \mathrm{d} s_{1} \int \mathrm{d} s_{2} R_{2}\left(s_{1}, \operatorname{MAPI}(5)\right) R_{2}\left(s_{2}, \operatorname{MAPI}(4)\right) R_{2}(s, \operatorname{MAPI}(3)) \\
\int \mathrm{d} \Phi^{(5)}= & \int \mathrm{d} s_{1} \int \mathrm{d} s_{2} \int \mathrm{d} s_{3} R_{2}\left(s_{1}, \operatorname{MAPIG}(5)\right) \\
& \times R_{2}\left(s_{2}, \operatorname{MAPIG}(4)\right) R_{2}\left(s_{3}, \operatorname{MAPIG}(3)\right) R_{2}(s, \operatorname{MAPIG}(8)) .
\end{aligned}
$$

The propagator mappings for the functions $R_{2}$ and for the variables $s_{1}, s_{2}, s_{3}$ are specified by the values of the variables MAPI and MAPIG for the four- and five-particle phase spaces, respectively. In this way all channels for the multi-channel integration of the processes $\mathrm{e}^{+} \mathrm{e}^{-} \rightarrow 4 f$ and $\mathrm{e}^{+} \mathrm{e}^{-} \rightarrow 4 f \gamma$ can be constructed from the two and three basic phase-space decompositions, respectively, shown in Figure 3. Note that several topologies of Figure 2 fit into single structural diagrams of Figure 3. The definitions of the variables $s_{i}$ and their phase-space boundaries are given in Table 1.

The phase-space generators of individual channels are built generically from these basic phase-space decompositions in kern. $f$ by calling subroutines PHSPGEN_4 or PHSPGEN_5:

SUBROUTINE PHSPGEN_4(IDENS, II , MAPI , VZI , NU , S , X , WPS , ICUT)

SUBROUTINE PHSPGEN_5(IDENS, IIG, MAPIG , VZIG , NU, S , X , WPS , ICUT)

IDENS $\quad:$ determines whether the four-momenta $k_{i}($ IDENS $=0)$ or the density $g_{k}$ is calculated (IDENS $=1)$,

II IIG : II (1:4),IIG(1:5) specify the permutations of the final-state particles, II, IIG $=1, \ldots, 4$ denote the final-state fermions in the order of the input file, $\mathrm{IIG}=5$ denotes the photon, 


\begin{tabular}{|l|l|c|}
\hline topology & invariants & phase-space boundaries \\
\hline \hline MAPI $(6)=1$ & $s_{1}=\left(k_{\mathrm{II}(1)}+k_{\mathrm{II}(2)}\right)^{2}$ & $0 \leq s_{1} \leq s$ \\
& $s_{2}=\left(k_{\mathrm{II}(3)}+k_{\mathrm{II}(4)}\right)^{2}$ & $0 \leq s_{2} \leq\left(\sqrt{s}-\sqrt{s_{1}}\right)^{2}$ \\
\hline $\operatorname{MAPI}(6)=2$ & $s_{1}=\left(k_{\mathrm{II}(1)}+k_{\mathrm{II}(2)}\right)^{2}$ & $0 \leq s_{1} \leq s$ \\
& $s_{2}=\left(k_{\mathrm{II}(1)}+k_{\mathrm{II}(2)}+k_{\mathrm{II}(3)}\right)^{2}$ & $s_{1} \leq s_{2} \leq s$ \\
\hline \hline $\operatorname{MAPIG}(6)=1$ & $s_{1}=\left(k_{\mathrm{IIG}(1)}+k_{\mathrm{IIG}(2)}\right)^{2}$ & $0 \leq s_{1} \leq s$ \\
& $s_{2}=\left(k_{\mathrm{IIG}(1)}+k_{\mathrm{IIG}(2)}+k_{\mathrm{IIG}(3)}\right)^{2}$ & $s_{1} \leq s_{2} \leq s$ \\
& $s_{3}=\left(k_{\mathrm{IIG}(4)}+k_{\mathrm{IIG}(5)}\right)^{2}$ & $0 \leq s_{3} \leq\left(\sqrt{s}-\sqrt{s_{2}}\right)^{2}$ \\
\hline $\operatorname{MAPIG}(6)=2$ & $s_{1}=\left(k_{\mathrm{IIG}(1)}+k_{\mathrm{IIG}(2)}\right)^{2}$ & $0 \leq s_{1} \leq s$ \\
& $s_{2}=\left(k_{\mathrm{IIG}(3)}+k_{\mathrm{IIG}(4)}\right)^{2}$ & $0 \leq s_{2} \leq\left(\sqrt{s}-\sqrt{s_{1}}\right)^{2}$ \\
& $s_{3}=\left(k_{\mathrm{IIG}(1)}+k_{\mathrm{IIG}(2)}+k_{\mathrm{IIG}(3)}+k_{\mathrm{IIG}(4)}\right)^{2}$ & $\left(\sqrt{s_{1}}+\sqrt{s_{2}}\right)^{2} \leq s_{3} \leq s$ \\
\hline $\operatorname{MAPIG}(6)=3$ & $s_{1}=\left(k_{\mathrm{IIG}(1)}+k_{\mathrm{IIG}(2)}\right)^{2}$ & $0 \leq s_{1} \leq s$ \\
& $s_{2}=\left(k_{\mathrm{IIG}(1)}+k_{\mathrm{IIG}(2)}+k_{\mathrm{IIG}(3)}\right)^{2}$ & $s_{1} \leq s_{2} \leq s$ \\
& $s_{3}=\left(k_{\mathrm{IIG}(1)}+k_{\mathrm{IIG}(2)}+k_{\mathrm{IIG}(3)}+k_{\mathrm{IIG}(4)}\right)^{2}$ & $s_{2} \leq s_{3} \leq s$ \\
\hline
\end{tabular}

Table 1

The definition of the invariants $s_{i}$ in Eqs. (22) and (23) and their phase-space boundaries for the different topologies in the slicing branch

MAPI , MAPIG $: \operatorname{MAPI}(6)=1,2$ and $\operatorname{MAPIG}(6)=1,2,3$ numerate the basic topologies,

$\operatorname{MAPI}(1: 2), \operatorname{MAPIG}(1: 2)$, and MAPIG(7) specify the $s$-channel propagator mappings of the variables $s_{1}, s_{2}, s_{3}$ of Eqs. (22) and (23):

MAPI, MAPIG $=0:$ no mapping,

MAPI, MAPIG $=1$ : mapping for a massless propagator Eq. (9) or Eq. (10),

MAPI, MAPIG $=2:$ mapping for a W-boson propagator Eq. (7),

MAPI, MAPIG $=3:$ mapping for a Z-boson propagator Eq. (7),

MAPI (3:5), MAPIG(3:5), and MAPIG(8) specify the $t$-channel propagator mappings in the functions $R_{2}$ of Eqs. (22) and (23):

MAPI, MAPIG $=0:$ no mapping $(s$-channel $2 \rightarrow 2$ scattering $)$,

MAPI, MAPIG $=1$ : no mapping (massive particle in $t$-channel propagator),

MAPI, MAPIG $=2:$ mapping for a massless $t$-channel propagator

using Eq. (9) or Eq. (10), 


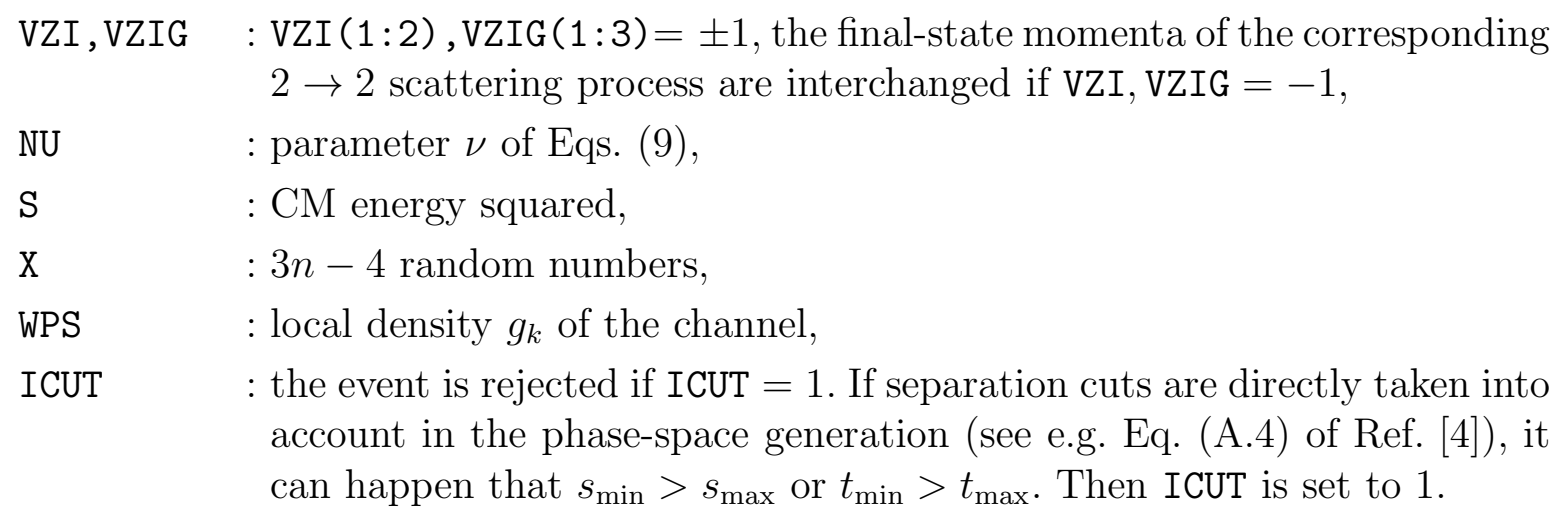
account in the phase-space generation (see e.g. Eq. (A.4) of Ref. [4]), it can happen that $s_{\min }>s_{\max }$ or $t_{\min }>t_{\max }$. Then ICUT is set to 1 .

The channels for $4 f$ and $4 f \gamma$ production are obtained from the basic topologies by specifying the parameters II,MAPI,VZI and IIG, MAPIG, VZIG, respectively. The parameters VZI, VZIG distinguish between $t$-channel processes $q_{1}+q_{2} \rightarrow p_{1}+p_{2}$ with invariants $t=q_{1}-p_{1}$ or $t=q_{1}-p_{2}$. For instance, the topology of Figure 1 where the final-state particle with momentum $k_{6}$ is emitted from the electron instead of the positron is obtained by changing the sign of VZIG(1). The specifications of the relevant parameters for each channel for a given final state is done in the subroutine ADDMAP in slicing.f. For the example of the multi-peripheral diagram given in Ref. [4], the multi-channel parameters are MAPIG $(1: 8)=\{1,3,2,0,0,3,0,2\}, \operatorname{IIG}(1: 5)=\{3,5,4,2,1\}$, and $\operatorname{VZIG}(1: 2)=\{1,-1\}$ (VZIG(3) is not used for $\operatorname{MAPIG}(4)=0$ topologies).

The propagator mappings specified by the parameters MAPI and MAPIG are performed according to Eqs. (7)-(10) in kern.f by the subroutines SMAP and TMAP, respectively. The corresponding Jacobians (WPSI) are calculated in the subroutines SDENS and TDENS. The Lorentz transformations used in the calculation of the outgoing momenta consist of a combination of boosts along the $z$ direction and rotations described by the subroutines BOOST_Z and ROTATION in kern.f.

\subsubsection{Phase-space generation in the subtraction branch}

In the subtraction branch, the generation of the momenta of the final-state particles and the calculation of the local densities are done in

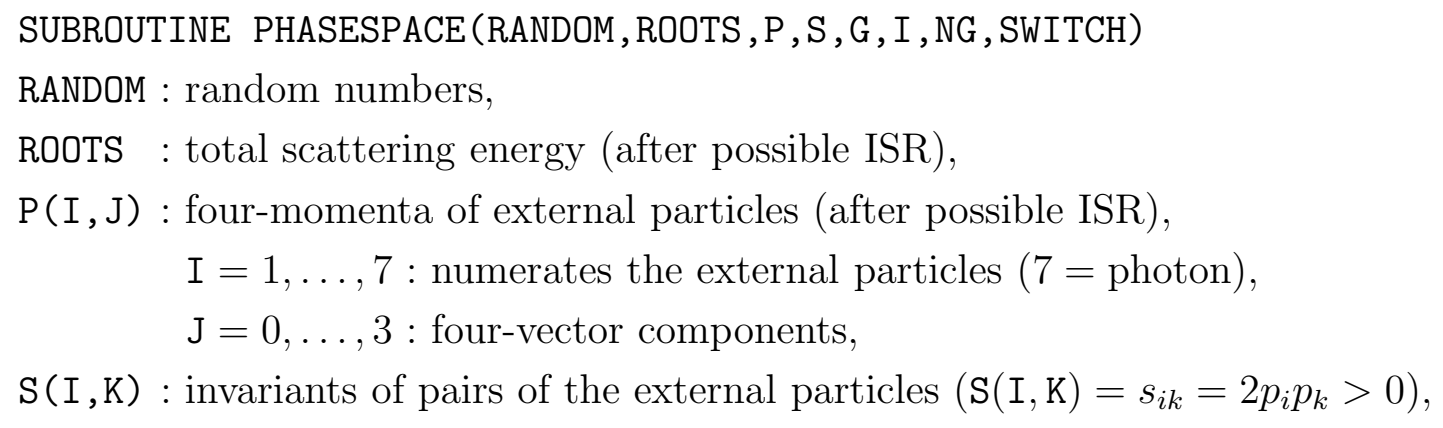


$\mathrm{I}, \mathrm{K}=1, \ldots, 7:$ numerate the external particles $(7=$ photon $)$,

G : local density $g_{i}$ of the channel $I$,

I : number of the channel,

NG : number of the phase-space generator,

SWITCH : decides between event generation, calculation of the local density, and initialization of the generator,

SWITCH $=0:$ an error occurred (event will be rejected),

SWITCH $=1$ : generation of momenta and invariants,

SWITCH $=2:$ calculation of the local density of the given channel,

SWITCH $=3:$ checks whether the diagram exists (initialization),

SWITCH $=4:$ resulting flag if the diagram exists

in subtraction.f. The subroutine PHASESPACE is called several times in the subroutine SUBTRACTION where the weights of the different subcontributions are calculated. Three different phase-space generators are used:

$\mathrm{NG}=1$ : four-particle phase-space generator used for tree-level process $\mathrm{e}^{+} \mathrm{e}^{-} \rightarrow 4 f$ (including optionally higher-order LL corrections from ISR) or for the IBA to the processes $\mathrm{e}^{+} \mathrm{e}^{-} \rightarrow \mathrm{WW} \rightarrow 4 f$,

$\mathrm{NG}=2$ : four-particle phase-space generator for the $4 f$ part of $\varnothing(\alpha)$ radiative corrections,

$\mathrm{NG}=3$ : five-particle phase-space generator used for the $4 f \gamma$ part of the $\varnothing(\alpha)$ radiative corrections, for the tree-level process $\mathrm{e}^{+} \mathrm{e}^{-} \rightarrow 4 f \gamma$, or for the IBA to the processes $\mathrm{e}^{+} \mathrm{e}^{-} \rightarrow 4 f \gamma$.

The subroutine PHASESPACE is decomposed into 15 parts which correspond to the different topologies contributing to the processes $\mathrm{e}^{+} \mathrm{e}^{-} \rightarrow 4 f$ and $\mathrm{e}^{+} \mathrm{e}^{-} \rightarrow 4 f \gamma$. There are 5 topologies for the processes $\mathrm{e}^{+} \mathrm{e}^{-} \rightarrow 4 f$ and 10 topologies for $\mathrm{e}^{+} \mathrm{e}^{-} \rightarrow 4 f \gamma$, as shown in Figure 2 .

The initialization of the channels is done by calling

SUBROUTINE INITPHASESPACE (NUPS, Q, NG, NPARTICLE, INPUTONSHELL, SRC , SIGNAL , NOCUTS)

NUPS $\quad:$ parameter $\nu$ used in the propagator mapping of Eq. (9),

Q(I) : charges of the external fermions $(I=1, \ldots, 6)$,

NG : number of the phase-space generator,

NPARTICLE : number of final-state particles (NPARTICLE $=4,5$ ),

INPUTONSHELL : if INPUTONSHELL $=1$ the phase space is generated for on-shell $\mathrm{W}$ bosons,

SRC : include special channels for the subtraction functions of the $4 f \gamma$ part of the radiative corrections for $\mathrm{SRC}=1$ and NPARTICLE $=5$, 
SIGNAL : restricts to subsets of diagrams as given in Table 4,

NOCUTS $\quad$ decides whether cuts are included in the event generation $($ NOCUTS $=0)$ or not $($ NOCUTS $=1)$

in subtraction.f. The subroutine INITPHASESPACE for the three phase-space generators is called in INITSUBTRACTION in subtraction.f, where the options for the phase-space generators and for different branches can be found.

To initialize the phase-space generators, SEARCHGENERATOR (which is just a second entry of PHASESPACE) is called in INITPHASESPACE with SWITCH $=3$. If the diagram of the tested channel exists, SEARCHGENERATOR yields SWITCH $=4$, and the channel is used in the phase-space generation. The actual selection of the channels is done by inserting the external particles EP1, ., EP7 (and the emitter EM and spectator SP for special channels related to subtraction functions) and the virtual particles IP1, ., IP4 in all possible ways, and testing whether the vertices of the topological diagram exist or not. This is done in the functions V3 and V4 for the three- and four-particle vertices, respectively. The vertices of the SM are defined in the functions V3G and V4G. Some additional criteria are added to select subsets of diagrams (CHECKSIGNAL), and to avoid double-counting of equivalent channels, i.e. channels which have the same propagator mappings. To speed up the initialization, the virtual particles are inserted one after another, and as soon as a vertex does not exist the diagram is discarded. All informations of the phase-space generators are stored in the common block CHANNELS in subtraction.f.

For $\operatorname{SWITCH}=1$ the event is generated, i.e. the momenta of the final-state particles are calculated from the random numbers RANDOM, the incoming momenta $P(1: 2,0: 3)$, and the scattering energy ROOTS. For SWITCH $=2$ the local density $G$ of the channel $I$ is calculated from momenta $\mathrm{P}$, invariants $\mathrm{S}$, and from ROOTS. The invariants $\mathrm{S}$ are calculated in the subroutine SDET once per event for all local densities. The actual calculations are done by calling the subroutines SPROP, DECAY, and PROCESS in subtraction.f:

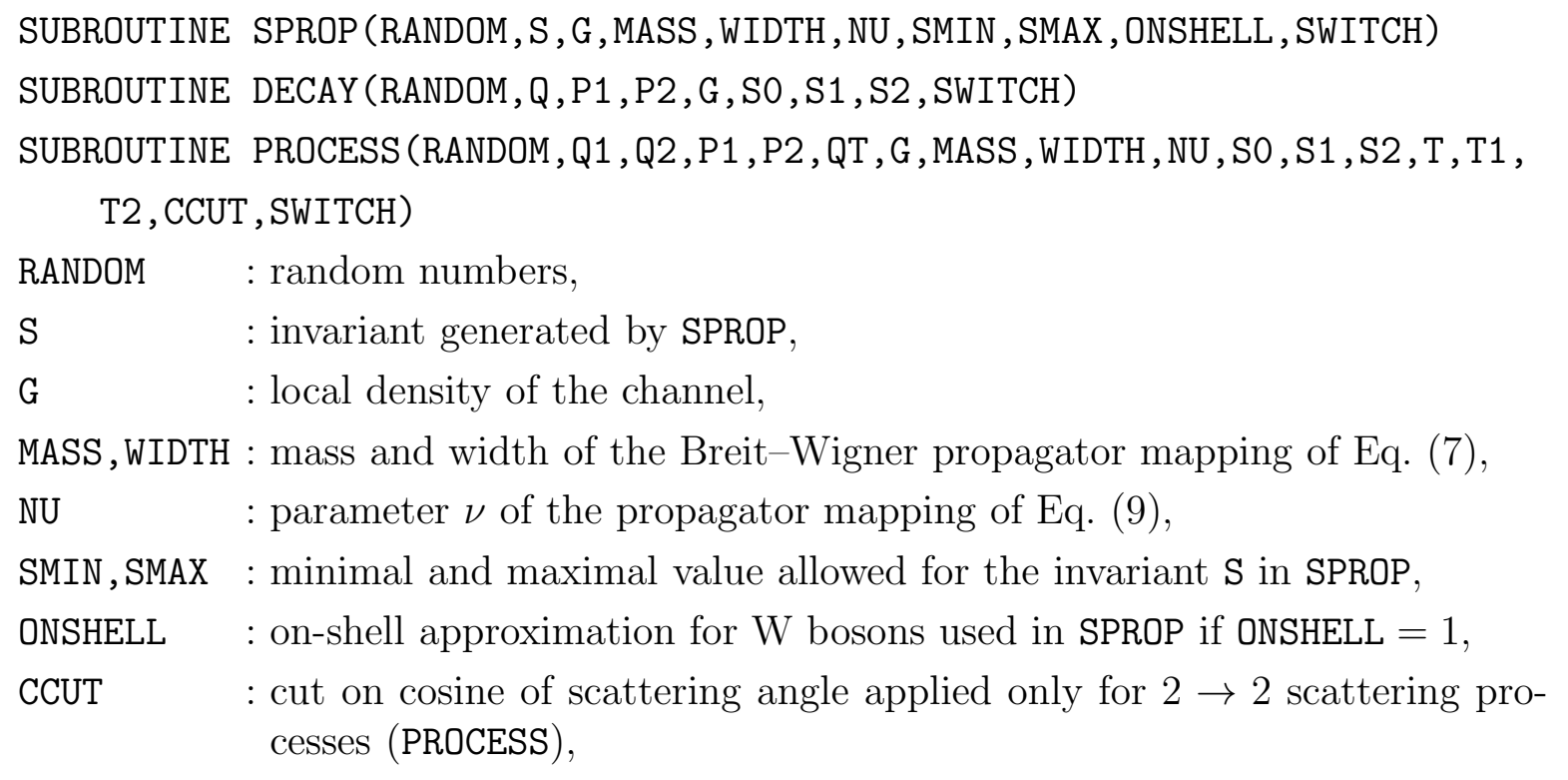


SUBROUTINE PROCESS :

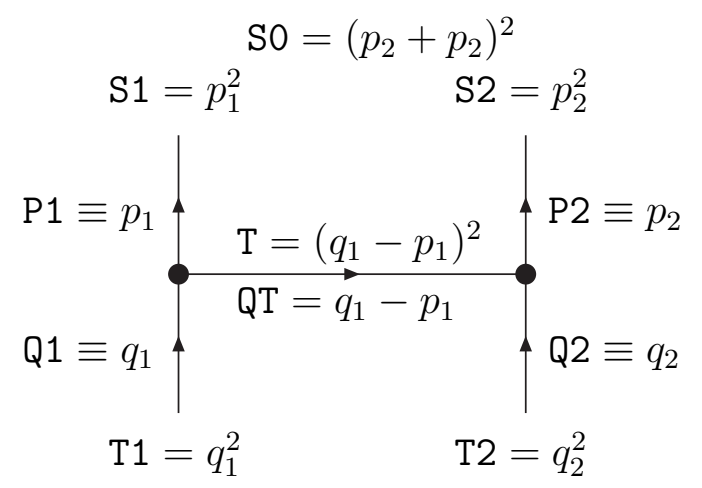

SUBROUTINE DECAY:

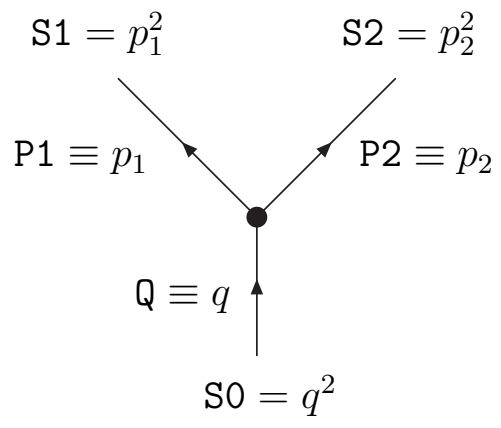

Fig. 4. The generic diagrams of the $2 \rightarrow 2$ scattering process and $1 \rightarrow 2$ decay and the arguments of the corresponding subroutines PROCESS and DECAY in subtraction.f

SWITCH : see subroutine PHASESPACE,

the remaining arguments are given in Figure 4.

The propagator mappings of the massless propagators of Eqs. (7) and (9) and the calculation of the corresponding Jacobians are done by calling the functions $\mathrm{H}$ and JACOBIAN, respectively, in the subroutines SPROP and PROCESS in subtraction.f. For the boosts and rotations of momenta the subroutines BOOST and ROTATION in subtraction.f are used.

For the subtraction functions corresponding to the bremsstrahlung process $\mathrm{e}^{+} \mathrm{e}^{-} \rightarrow 4 f \gamma$, special channels are included in the calculation to improve the numerical stability. Since these involve the Born cross section to $\mathrm{e}^{+} \mathrm{e}^{-} \rightarrow 4 f$, a mapping of the $4 f \gamma$ phase space into the $4 f$ phase space is needed. The mapping from the five- to the four-particle phase space and back is performed by calling the subroutines MAP54 and MAP45 in PHASESPACE, respectively. The propagators of the Born process $\mathrm{e}^{+} \mathrm{e}^{-} \rightarrow 4 f$ are then smoothened by the four-particle phase-space generator. Additional mappings implemented in MAP54 and MAP45 account for the smoothening of the remaining peaking behaviour of the subtraction functions, i.e. of the splitting functions.

\subsection{The matrix-element evaluation}

In this section, we describe the matrix-element evaluation in the three different modes of RACOONWW. Furthermore, we briefly sketch the treatment of higher-order ISR and QCD corrections, and the implementation of anomalous gauge-boson couplings. 


\section{Table 2}

\begin{tabular}{|c|c|c|c|c|c|c|c|}
\hline \multicolumn{8}{|c|}{$\mathrm{CC}$ processes: $\mathrm{CC}=1, \mathrm{NC}=0$} \\
\hline & $\overline{f_{1}}$ & $f_{2}$ & $\rightarrow$ & $f_{3}$ & $\bar{f}_{4}$ & $f_{5}$ & $\bar{f}_{6}$ \\
\hline $\mathrm{SYM}=0$ & $\mathrm{e}^{+}$ & $\mathrm{e}^{-}$ & $\rightarrow$ & & $\overline{f^{\prime}}$ & $F$ & $\bar{F}^{\prime}$ \\
\hline $\mathrm{SYM}=1$ & $\mathrm{e}^{+}$ & $\mathrm{e}^{-}$ & $\rightarrow$ & $\nu_{e}$ & $\mathrm{e}^{+}$ & $f$ & $\bar{f}^{\prime}$ \\
\hline $\mathrm{SYM}=2$ & $\mathrm{e}^{+}$ & $\mathrm{e}^{-}$ & $\rightarrow$ & $f$ & $\bar{f}^{\prime}$ & $\mathrm{e}^{-}$ & $\bar{\nu}_{e}$ \\
\hline \multicolumn{8}{|c|}{ NC processes: $\mathrm{CC}=0, \mathrm{NC}=1$} \\
\hline & $\overline{f_{1}}$ & $f_{2}$ & $\rightarrow$ & $f_{3}$ & $\bar{f}_{4}$ & $f_{5}$ & $\bar{f}_{6}$ \\
\hline $\mathrm{SYM}=0$ & $\overline{\mathrm{e}^{+}}$ & $\overline{\mathrm{e}^{-}}$ & $\rightarrow$ & $f$ & $\bar{f}$ & $F$ & $\bar{F}$ \\
\hline $\mathrm{SYM}=1$ & $\mathrm{e}^{+}$ & $\mathrm{e}^{-}$ & $\rightarrow$ & $f$ & $\bar{f}$ & $f$ & $\bar{f}$ \\
\hline $\mathrm{SYM}=2$ & $\mathrm{e}^{+}$ & $\mathrm{e}^{-}$ & $\rightarrow$ & $\mathrm{e}^{-}$ & $\mathrm{e}^{+}$ & $f$ & $\bar{f}$ \\
\hline $\mathrm{SYM}=3$ & $\mathrm{e}^{+}$ & $\mathrm{e}^{-}$ & $\rightarrow$ & $\mathrm{e}^{-}$ & $\mathrm{e}^{+}$ & $\mathrm{e}^{-}$ & $\mathrm{e}^{+}$ \\
\hline \multicolumn{8}{|c|}{ Mixed $(\mathrm{CC} \& \mathrm{NC})$ processes: $\mathrm{CC}=1, \mathrm{NC}=1$} \\
\hline & $\bar{f}_{1}$ & $f_{2}$ & $\rightarrow$ & $f_{3}$ & $\bar{f}_{4}$ & $f_{5}$ & $\bar{f}_{6}$ \\
\hline $\mathrm{SYM}=0$ & $\mathrm{e}^{+}$ & $\mathrm{e}^{-}$ & $\rightarrow$ & $f$ & $\bar{f}$ & $f^{\prime}$ & $\bar{f}^{\prime}$ \\
\hline$=1$ & $\mathrm{e}^{+}$ & $\mathrm{e}^{-}$ & $\rightarrow$ & $\nu_{e}$ & $\bar{\nu}_{e}$ & $f$ & $\bar{f}$ \\
\hline SYM & $\mathrm{e}^{+}$ & $\mathrm{E}$ & $\rightarrow$ & $\nu_{e}$ & $\bar{\nu}_{e}$ & $\nu_{e}$ & $\bar{\nu}_{e}$ \\
\hline $\mathrm{SYM}=3$ & $\mathrm{e}^{+}$ & $\mathrm{e}^{-}$ & $\rightarrow$ & $\nu_{e}$ & $\bar{\nu}_{e}$ & $\mathrm{e}^{-}$ & $\mathrm{e}^{+}$ \\
\hline
\end{tabular}

The classification of the four-fermion processes $\mathrm{e}^{+} \mathrm{e}^{-} \rightarrow 4 f$. Here, $f$ and $F$ denote different fermions $(f \neq F)$ that are neither electrons nor electron neutrinos $\left(f, F \neq \mathrm{e}^{-}, \nu_{\mathrm{e}}\right)$, and their weak-isospin partners are denoted by $f^{\prime}$ and $F^{\prime}$, respectively. The parameters SYM, NC, and CC are determined in the subroutine INIT_EPEM in ee4fa_amps.f.

\subsubsection{The tree-level mode $(\mathrm{SRC}=0)$}

In the tree-level mode $(\mathrm{SRC}=0)$ RACOONWW calculates either the processes $\mathrm{e}^{+} \mathrm{e}^{-} \rightarrow 4 f$ $(\operatorname{SBORN} 4=1,2,3)$ or the radiative processes $\mathrm{e}^{+} \mathrm{e}^{-} \rightarrow 4 f \gamma(\mathrm{SBORN5}=1)$ at lowest order for all possible four-fermion final states. The flags SBORN4 and SBORN5 are set in the input file. The Coulomb singularity to the tree-level process $\mathrm{e}^{+} \mathrm{e}^{-} \rightarrow 4 f$ can be included by setting SCOULTREE $=1$, which corresponds to the variant 1 of Section 3.2.2. The treatment of the Coulomb singularity in $\mathrm{e}^{+} \mathrm{e}^{-} \rightarrow 4 f \gamma$ processes is described in Section 3.2.2.

The different process classes are characterized according to the appearing intermediate vector bosons, i.e. whether the reactions proceed via charged-current (CC), or neutralcurrent (NC) interactions, or via both interaction types (see Table 2). The classification can be performed by considering the quantum numbers of the final-state fermion pairs. As shown in Table 2, we identified 11 classes of processes. This classification does not change when a photon is radiated. In the following, we often use the shorthands CC11 and CC03. CC11 denotes the set of up to 11 Feynman diagrams contributing to the simplest CC process in Table $2(\mathrm{SYM}=0)$, which is the smallest gauge-invariant subset of diagrams including $\mathrm{W}$-pair production. CC03 denotes the 3 diagrams that include 2 resonant $\mathrm{W}$ bosons, which is a non-gauge-invariant subset of CC11 (see also Ref. [10]).

The calculation of the matrix elements to the tree-level processes $\mathrm{e}^{+} \mathrm{e}^{-} \rightarrow 4 f$ and $\mathrm{e}^{+} \mathrm{e}^{-} \rightarrow$ 
a) Abelian graph

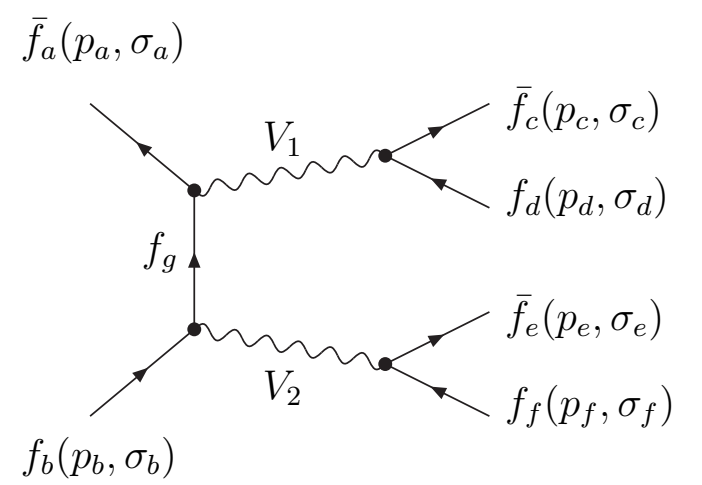

b) Non-abelian graph

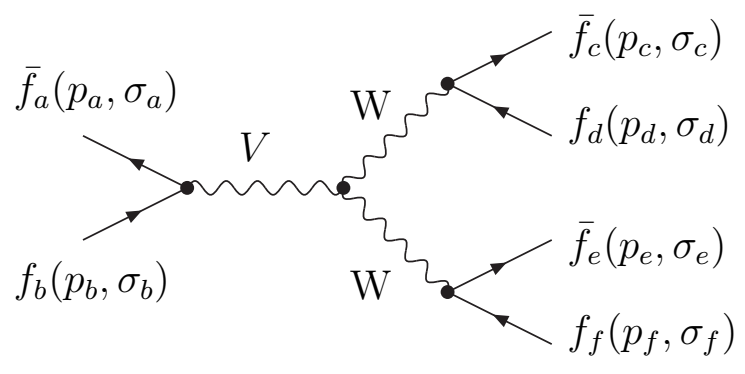

Fig. 5. Generic diagrams for $\mathrm{e}^{+} \mathrm{e}^{-} \rightarrow 4 f$

$4 f \gamma$ has been described in detail in Ref. [4]. For $\mathrm{e}^{+} \mathrm{e}^{-} \rightarrow 4 f$ the matrix elements of all Feynman diagrams can been reduced to two generic functions that are related to the two basic graphs shown in Figure 5. For $\mathrm{e}^{+} \mathrm{e}^{-} \rightarrow 4 f$, the calculation is similar to the one in Ref. [27]. For $\mathrm{e}^{+} \mathrm{e}^{-} \rightarrow 4 f \gamma$, all graphs that are obtained from one of the generic graphs in Figure 5 by attaching one additional real photon in all possible ways are combined into one generic function. Finally, also all amplitudes to $\mathrm{e}^{+} \mathrm{e}^{-} \rightarrow 4 f \gamma$ can be constructed from only two generic functions. For the helicity amplitudes corresponding to the generic contributions, concise results have been obtained by using the Weyl-van der Waerden (WvdW) formalism (see Ref. [28] and references therein).

The helicity amplitudes to $\mathrm{e}^{+} \mathrm{e}^{-} \rightarrow 4 f$ are calculated in the function M2_EPEM_4F by calling either the subroutine M_CC or M_NC or both, depending on the process under consideration:

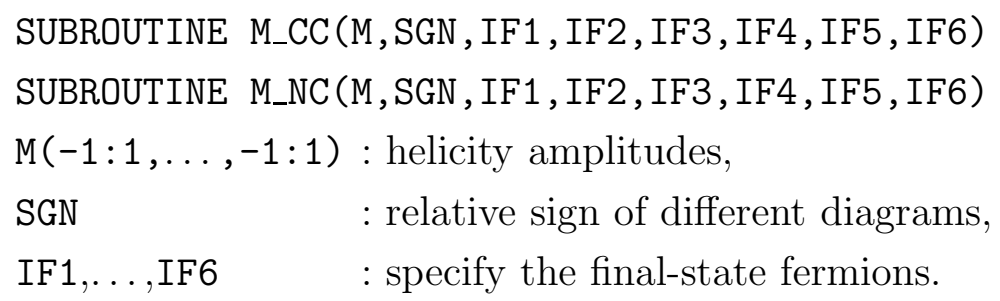

The helicity amplitudes are expressed in terms of WvdW spinor products, which are calculated in the subroutine SETPRODS. Note that SETPRODS must to be called before the matrix elements are evaluated by the subroutines M2_EPEM_4F or M2_EPEM_4FA. The helicity amplitudes for a four-fermion final state, $\mathrm{M}$, are built up by using two generic functions related to the graphs of Figure 5 as calculated in the subroutines ADDMVV for the abelian diagram and ADDMVVV for the non-abelian diagram of Figure 5, respectively, with the appropriate choices for the parameters SGN, IF $1, \ldots, I F 6$, and by specifying the intermediate gauge bosons $V, V_{1}, V_{2}$ of Figure 5 by the variables IV, IV1, IV2, which are arguments of ADDMVV and ADDMVVV.

The generic functions for $\mathrm{e}^{+} \mathrm{e}^{-} \rightarrow 4 f \gamma$ can be constructed in a similar way. The helicity amplitudes to $\mathrm{e}^{+} \mathrm{e}^{-} \rightarrow 4 f \gamma$ are built up in the subroutines M_CCA and M_NCA. Again the contributions of all diagrams can be taken into account by calling the corresponding subroutines ADDMAVV and ADDMAVVV and making the appropriate choices for the parameters 
SGN, IF1,..,IF6, IV, IV1, IV2.

For the $\mathrm{NC}(\mathrm{SYM}=0,1)$ and $\mathrm{CC} / \mathrm{NC}(\mathrm{SYM}=0)$ type of processes with pure hadronic final states, one of the gauge bosons in the diagram of Figure 5 can be a gluon. In RACOONWW these contributions are taken into account when choosing SQCDEPEM $=1$ in the input file for both processes $\mathrm{e}^{+} \mathrm{e}^{-} \rightarrow 4 f$ and $\mathrm{e}^{+} \mathrm{e}^{-} \rightarrow 4 f \gamma$. Then, the complete matrix elements for the fully hadronic channels result from the sum of the purely electroweak and the gluonexchange contributions. The contribution of the gluon-exchange diagrams are calculated in the subroutines MG_NC and MG_NCA for the process classes $\mathrm{e}^{+} \mathrm{e}^{-} \rightarrow 4 f$ and $\mathrm{e}^{+} \mathrm{e}^{-} \rightarrow 4 f \gamma$, respectively.

\subsubsection{The improved-Born approximation (IBA) mode $(\mathrm{SRC}=2,3)$}

RACOONWW provides an IBA for the process $\mathrm{e}^{+} \mathrm{e}^{-} \rightarrow \mathrm{WW} \rightarrow 4 f(\mathrm{SRC}=2)$ including only diagrams of the CC03 class, and to the radiative processes $\mathrm{e}^{+} \mathrm{e}^{-} \rightarrow 4 f \gamma(\mathrm{SRC}=3)$, as described in Refs. [7] and [8], respectively. For both process classes universal leading electroweak corrections are taken into account. Universal radiative corrections are corrections that are either connected to specific important subprocesses, such as collinear photon emission, or that originate from large corrections related to renormalization effects, like running couplings, and involve characteristic enhancement factors. Owing to their universality such corrections can be related in a simple way to the lowest-order matrix element of the underlying process.

Near the W-pair-production threshold, a particularly important correction is induced by the Coulomb singularity. This arises from diagrams where a soft photon is exchanged between two nearly on-shell $\mathrm{W}$ bosons close to their kinematical production threshold and results in a simple factor that depends on the momenta $k_{ \pm}$of the $\mathrm{W}^{ \pm}$bosons $[17,29]$,

$$
\begin{aligned}
\mathrm{d} \sigma_{\mathrm{Coul}} & =\left[1+\delta_{\mathrm{Coul}}\left(s, k_{+}^{2}, k_{-}^{2}\right) g(\bar{\beta})\right] \mathrm{d} \sigma_{\mathrm{Born}}, \\
\delta_{\mathrm{Coul}}\left(s, k_{+}^{2}, k_{-}^{2}\right) & =\frac{\alpha(0)}{\bar{\beta}} \operatorname{Im}\left[\ln \left(\frac{\beta-\bar{\beta}+\Delta_{M}}{\beta+\bar{\beta}+\Delta_{M}}\right)\right], \\
\bar{\beta} & =\frac{\sqrt{s^{2}+k_{+}^{4}+k_{-}^{4}-2 s k_{+}^{2}-2 s k_{-}^{2}-2 k_{+}^{2} k_{-}^{2}}}{s}, \\
\beta & =\sqrt{1-\frac{4\left(M_{\mathrm{W}}^{2}-\mathrm{i} M_{\mathrm{W}} \Gamma_{\mathrm{W}}\right)}{s}}, \quad \Delta_{M}=\frac{\left|k_{+}^{2}-k_{-}^{2}\right|}{s},
\end{aligned}
$$

with the fine-structure constant $\alpha(0)$, and $s=\left(k_{+}+k_{-}\right)^{2}$. The auxiliary function

$$
g(\bar{\beta})=\left(1-\bar{\beta}^{2}\right)^{2}
$$

is introduced to restrict the impact of $\delta_{\text {Coul }}$ to the threshold region.

A further ingredient in the IBA is the LL contribution induced by ISR. This is included by 
using the structure-function approach [18], where the full IBA matrix elements squared to $\mathrm{e}^{+} \mathrm{e}^{-} \rightarrow \mathrm{WW} \rightarrow 4 f$ and $\mathrm{e}^{+} \mathrm{e}^{-} \rightarrow 4 f \gamma$ are multiplied by the ISR structure functions. Their scale dependence can be used to adjust the IBA to the full correction, but also to estimate the intrinsic uncertainty of the IBA by choosing different values for $Q$.

When the IBA to $\mathrm{e}^{+} \mathrm{e}^{-} \rightarrow \mathrm{WW} \rightarrow 4 f$ is calculated, the (off-shell) improved Born matrix element squared described by

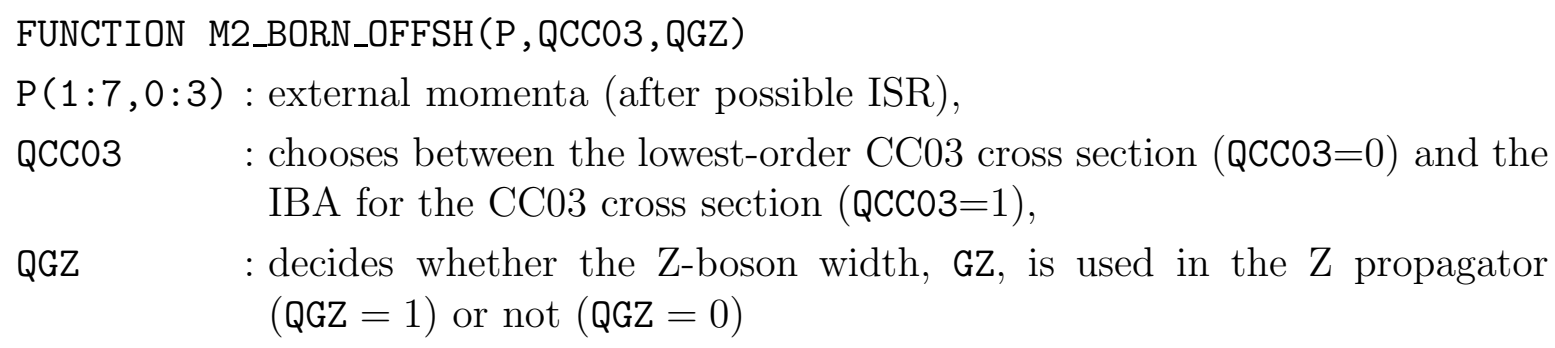

in eeWW4f_amps.f is convoluted with the ISR structure functions. The Born matrix element includes all contributions from the CC03 class of diagrams (QCC03 =1) which have been modified in such a way that the universal renormalization effects induced by the running of $\alpha$ and by $\Delta \rho$ are absorbed. This is achieved [30] by the replacements

$$
\frac{e^{2}}{s_{\mathrm{w}}^{2}} \rightarrow 4 \sqrt{2} G_{\mu} M_{\mathrm{W}}^{2}, \quad e^{2} \rightarrow 4 \pi \alpha(s)
$$

in the amplitudes, which implies that weak-isospin exchange involves the coupling $G_{\mu} M_{\mathrm{W}}^{2}$ and pure photon exchange the coupling $\alpha(s)$. The running of the electromagnetic coupling is induced by light (massless) charged fermions only, i.e. we evaluate $\alpha(s)$ by

$$
\alpha(s)=\frac{\alpha\left(M_{\mathrm{Z}}^{2}\right)}{1-\frac{\alpha\left(M_{\mathrm{Z}}^{2}\right)}{3 \pi} \ln \left(\frac{s}{M_{\mathrm{Z}}^{2}}\right) \sum_{f \neq \mathrm{t}} N_{f}^{\mathrm{c}} Q_{f}^{2}},
$$

where $\alpha\left(M_{\mathrm{Z}}^{2}\right)=$ ALPHAZ is set in the subroutine PARAMETER in public.f. Furthermore, the Coulomb singularity is taken into account, and naive QCD corrections can be optionally included. The W-boson width is treated in the constant-width scheme. The complex Zboson mass is used $(\mathrm{QGZ}=1)$ in the $\mathrm{Z}$ propagators in order to regularize the $\mathrm{Z}$ resonance below the $\mathrm{W}$-pair production threshold, which may be reached after ISR.

The IBA for $\mathrm{e}^{+} \mathrm{e}^{-} \rightarrow 4 f \gamma$ processes is based on the full matrix elements of the tree-level mode in the $G_{\mu}$ scheme. Moreover, leading ISR corrections are included via structure functions, and the naive QCD correction can be optionally included. Again, the constantwidth scheme is used. As the tree-level mode, the IBA is not restricted to a certain class of processes, and the subset of diagrams included is governed by the choice of the flags SSIGEPEM5 and SQCDEPEM in the input file. For $\mathrm{e}^{+} \mathrm{e}^{-} \rightarrow 4 f \gamma$ the naive implementation of the Coulomb singularity involves some freedom, since the momenta of the W bosons depend on the photon momentum in different ways for photon radiation off initial-state 
and final-state particles. To control this ambiguity the Coulomb factor can be evaluated in two different ways:

(1) In the first variant (SCOULTREE $=1$ ), the complete matrix element squared is multiplied with the Coulomb correction factor using $k_{+}=k_{3}+k_{4}$ and $k_{-}=k_{5}+k_{6}$. In this way the correct Coulomb correction is multiplied to all diagrams where the photon is radiated off initial-state particles.

(2) In the second variant (SCOULTREE $=2$ ) this prescription is improved by choosing the correct Coulomb factor to all contributions involving the most resonant $\mathrm{W}^{+}$and $\mathrm{W}^{-}$bosons as determined from invariant masses in the final state. To this end, the W-boson momenta entering the Coulomb correction factor are fixed as

$$
\left(k_{+}, k_{-}\right)=\left\{\begin{array}{lll}
\left(k_{3}+k_{4}, k_{5}+k_{6}\right) & \text { for } \quad \Delta_{34}<\Delta_{347}, \Delta_{56}<\Delta_{567}, \\
\left(k_{3}+k_{4}+k_{7}, k_{5}+k_{6}\right) & \text { for } \quad \Delta_{34}>\Delta_{347}, \Delta_{56}<\Delta_{567} \text { or } \\
& & \Delta_{34}>\Delta_{347}, \Delta_{56}>\Delta_{567}, \Delta_{347}<\Delta_{567}, \\
\left(k_{3}+k_{4}, k_{5}+k_{6}+k_{7}\right) & \text { for } \quad \Delta_{34}<\Delta_{347}, \Delta_{56}>\Delta_{567} \text { or } \\
& & \Delta_{34}>\Delta_{347}, \Delta_{56}>\Delta_{567}, \Delta_{347}>\Delta_{567},
\end{array}\right.
$$

where $\Delta_{i j}=\left|\left(k_{i}+k_{j}\right)^{2}-M_{\mathrm{W}}^{2}\right|$ and $\Delta_{i j l}=\left|\left(k_{i}+k_{j}+k_{l}\right)^{2}-M_{\mathrm{W}}^{2}\right|$. Thus, the correct Coulomb correction factor is applied to all dominating doubly-resonant contributions.

Finally, for the IBA the $\mathrm{W}$-boson width $\Gamma_{\mathrm{W}}$ is calculated in lowest order using the $G_{\mu}$ scheme. This choice guarantees that the "effective branching ratios", which result after integrating out the decay parts, add up to one when summing over all channels. Of course, if naive QCD corrections are taken into account by multiplying with $\left(1+\alpha_{\mathrm{s}} / \pi\right)$ for each hadronically decaying $\mathrm{W}$ boson, for consistency these QCD factors are also included in the calculation of the total W-boson width.

\subsubsection{The radiative correction $(R C)$ mode $(\mathrm{SRC}=1)$}

In the $\mathrm{RC}$ mode $(\mathrm{SRC}=1)$ the complete electroweak corrections to $\mathrm{e}^{+} \mathrm{e}^{-} \rightarrow \mathrm{WW} \rightarrow 4 f$ in DPA are calculated as described in detail in Ref. [6]. Including $\mathcal{O}(\alpha)$ corrections, the cross section to $\mathrm{e}^{+} \mathrm{e}^{-} \rightarrow 4 f(\gamma)$ is composed of the following contributions

$$
\mathrm{d} \sigma=\mathrm{d} \sigma_{\text {Born }}^{\mathrm{e}^{+} \mathrm{e}^{-} \rightarrow 4 f}+\mathrm{d} \sigma_{\text {virt }}^{\mathrm{e}^{+} \mathrm{e}^{-} \rightarrow 4 f}+\mathrm{d} \sigma^{\mathrm{e}^{+} \mathrm{e}^{-} \rightarrow 4 f \gamma} .
$$

Here $\mathrm{d} \sigma_{\text {Born }}^{\mathrm{e}^{+} \mathrm{e}^{-} \rightarrow 4 f}$ and $\mathrm{d} \sigma^{\mathrm{e}^{+} \mathrm{e}^{-} \rightarrow 4 f \gamma}$ are the lowest-order cross sections to $\mathrm{e}^{+} \mathrm{e}^{-} \rightarrow 4 f$ and to $\mathrm{e}^{+} \mathrm{e}^{-} \rightarrow 4 f \gamma$, respectively, and $\mathrm{d} \sigma_{\text {virt }}^{\mathrm{e}^{+}} \mathrm{e}^{-} \rightarrow 4 f$ denotes the virtual one-loop corrections.

The $\mathrm{e}^{+} \mathrm{e}^{-} \rightarrow 4 f \gamma$ process (SBORN5 $=1$ ) represents the bremsstrahlung contribution, where the soft and collinear singularities are either subtracted from the matrix element squared (subtraction branch) or avoided by imposing technical cuts on the photon phase space (slicing branch). In the $\mathrm{RC}$ mode the calculation of the $\mathrm{e}^{+} \mathrm{e}^{-} \rightarrow 4 f \gamma$ process is always 
restricted to the CC11 class of diagrams (SSIGEPEM5 $=5$ ). Simultaneously, the tree-level process $\mathrm{e}^{+} \mathrm{e}^{-} \rightarrow 4 f($ SBORN4 $=1)$ can be calculated including all diagrams or only a subset of diagrams depending on the choice for SSIGEPEM4 in the input file.

Both the virtual and the real corrections involve soft and collinear singularities. These singularities are extracted by separating the cross sections into finite and singular parts:

$$
\begin{gathered}
\mathrm{d} \sigma_{\text {virt }}^{\mathrm{e}^{+} \mathrm{e}^{-} \rightarrow 4 f}=\mathrm{d} \sigma_{\text {virt,finite }}^{\mathrm{e}^{+} \mathrm{e}^{-} \rightarrow 4 f}+\mathrm{d} \sigma_{\text {virt,sing }}^{\mathrm{e}^{+} \mathrm{e}^{-} \rightarrow 4 f}, \\
\mathrm{~d} \sigma^{\mathrm{e}^{+} \mathrm{e}^{-} \rightarrow 4 f \gamma}=\mathrm{d} \sigma_{\text {finite }}^{\mathrm{e}^{+} \mathrm{e}^{-} \rightarrow 4 f \gamma}+\mathrm{d} \sigma_{\text {sing }}^{\mathrm{e}^{+} \mathrm{e}^{-} \rightarrow 4 f \gamma} .
\end{gathered}
$$

In RACOONWW, the DPA is only applied to the finite part of the virtual corrections,

$$
\mathrm{d} \sigma_{\text {virt,finite }}^{\mathrm{e}^{+} \mathrm{e}^{-} \rightarrow 4 f} \rightarrow \mathrm{d} \sigma_{\text {virt,finite,DPA }}^{\mathrm{e}^{+} \mathrm{e}^{-} \rightarrow \mathrm{WW} \rightarrow 4 f}=\mathrm{d} \sigma_{\text {virt,DPA }}^{\mathrm{e}^{+} \mathrm{e}^{-} \rightarrow \mathrm{WW} \rightarrow 4 f}-\mathrm{d} \sigma_{\text {virt,sing,DPA }}^{\mathrm{e}^{+} \mathrm{e}^{-} \rightarrow \mathrm{WW} \rightarrow 4 f} .
$$

The doubly-resonant virtual corrections are composed as

$$
\begin{aligned}
\mathrm{d} \sigma_{\text {virt,DPA }}^{\mathrm{e}^{+} \mathrm{e}^{-} \rightarrow \mathrm{WW} \rightarrow 4 f}= & \frac{1}{2 s} \mathrm{~d} \Phi_{4 f}\left\{2 \operatorname{Re}\left[\left(\mathcal{M}_{\text {Born,DPA }}^{\mathrm{e}^{+} \mathrm{e}^{-} \rightarrow \mathrm{WW} \rightarrow 4 f}\right)^{*} \delta \mathcal{M}_{\text {virt,fact,DPA }}^{\mathrm{e}^{+} \mathrm{e}^{-} \rightarrow \mathrm{WW} \rightarrow 4 f}\right]\right. \\
& \left.+\left|\mathcal{M}_{\text {Born,DPA }}^{\mathrm{e}^{+} \mathrm{e}^{-} \rightarrow \mathrm{WW} \rightarrow 4 f}\right|^{2} \delta_{\text {nfact,DPA }}^{\text {virt }}\right\}
\end{aligned}
$$

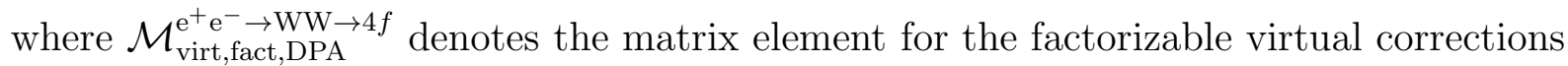
and $\delta_{\text {nfact,DPA }}^{\text {virt }}$ is the factor describing the non-factorizable virtual corrections. The matrix element squared to both contributions are calculated in eeWW4f_DPA.f by

FUNCTION M2(P,PON)

$P(1: 7,0: 3) \quad$ : four-momenta of external particles,

PON $(1: 7,0: 3)$ : four-momenta of external particles for on-shell $\mathrm{W}$ bosons.

The full four-fermion phase space (with off-shell $\mathrm{W}$ bosons) is generated, but the matrix elements for the DPA part are evaluated with kinematics for on-shell W bosons. This necessitates a mapping of the momenta of the final-state fermions such that the momenta of the $\mathrm{W}$ bosons become on shell. This is done in eeWW4f_DPA.f by

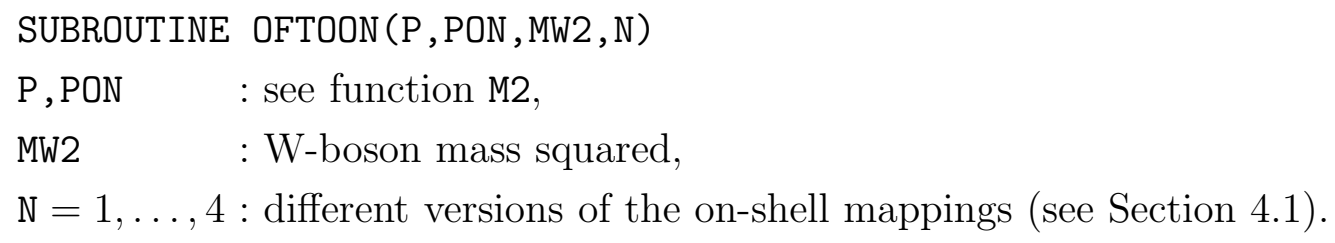

The factorizable contribution to the matrix element squared is calculated in the function DM2 $(F)$ in eeWW4f_amps. $f$ in terms of invariant coefficients $F(1: 10,-1: 1)$ which multiply 
so-called standard matrix elements, $\mathcal{M}_{n}^{\sigma}$, as $\square$

$$
\mathcal{M}_{\text {virt,fact,DPA }}^{\mathrm{e}^{+} \mathrm{e}^{-} \rightarrow \mathrm{WW} \rightarrow 4 f, \sigma}\left(\mathrm{p}_{+}, \mathrm{p}_{-}, \hat{k}_{+}, \hat{k}_{-}, k_{+}^{2}, k_{-}^{2}\right)=\sum_{n=1}^{10} F_{n}^{\sigma}(s, \hat{t}) \mathcal{M}_{n}^{\sigma}\left(\mathrm{p}_{+}, \mathrm{p}_{-}, \hat{k}_{+}, \hat{k}_{-}, k_{+}^{2}, k_{-}^{2}\right),
$$

where $\hat{k}_{ \pm}$are the on-shell momenta of the $\mathrm{W}^{ \pm}$bosons, and $\hat{t}=\left(p_{+}-\hat{k}_{+}\right)^{2}$. It is important to realize that the invariant coefficients $F_{n}^{\sigma}(s, \hat{t})=\mathrm{F}(n, \sigma)$ depend only on the scalar products of the momenta of the on-shell-projected $\mathrm{W}$ bosons, but not on the momenta of their decay products. For the default choice QREAL $=1$, the real part of $F$ is taken, i.e. only the real part of the factorizable virtual corrections is included. The form factors to $\mathrm{e}^{+} \mathrm{e}^{-} \rightarrow \mathrm{WW} \rightarrow 4 f$ are calculated in the subroutine CALCFORM for QFAST $=0$ and in FASTFORM for QFAST $=1$ in eeWW4f_DPA.f. The standard matrix elements are calculated in the subroutine CALCSME in eeWW4f_amps.f.

The actual numerical evaluation of the invariant coefficients $F_{n}^{\sigma}(s, \hat{t})$ can be rather CPUtime consuming, since the expressions for the one-loop contributions to these coefficients are rather involved (see Refs. [31,32]). Moreover, the employed Passarino-Veltman reduction [33] of tensor integrals to scalar integrals breaks down at the boundary of the phase space, necessarily leading to numerical instabilities in the very forward and backward directions of the $\mathrm{W}$-production angle $\theta$. In order to solve these problems of CPU time and numerical instabilities, RACOONWW uses as default (QFAST $=1$ ) a numerical reconstruction of the coefficients by making use of an expansion in Legendre polynomials $P_{l}(\cos \theta)$ :

$$
F_{n}^{\sigma}(s, \hat{t})=\sum_{l=0}^{\infty} \frac{1}{\hat{t}} c_{n, l}^{\sigma}(s) P_{l}(\cos \theta)
$$

which involves only simple algebra. Thus, its evaluation is extremely fast. In practice, relatively few generalized Fourier coefficients $c_{n, l}^{\sigma}(s)$ are needed; for example, taking $l=$ $0, \ldots, 20$ reproduces the full calculation of $F_{n}^{\sigma}(s, \hat{t})$ within roughly six digits for moderate scattering angles and LEP2 energies. The generalized Fourier coefficients are calculated via Gaussian integration during the initialization in the subroutine CFOURIER in eeWW4f_DPA.f. Note also that the generalized Fourier series remains stable in the forward and backward directions where the original evaluation of $F_{n}^{\sigma}(s, \hat{t})$ breaks down. The explicit factor $\hat{t}$ in Eq. (34) was included in order to account for a $t$-channel pole in some of the $F_{n}^{\sigma}(s, \hat{t})$; without this factor the expansion is less efficient and requires much more terms in the sum over $l$ in Eq. (34). The Legendre polynomials $P_{l}(\cos \theta)$ are calculated in eeWW4f_DPA.f by the subroutine LEGP.

1 In Ref. [6] the sum over $n$ runs from 1 to 7 , since the terms for $n=8,9,10$ could be expressed in terms of the first 7 terms. However, in RACOONWW the version with 10 invariant coefficients is used. 
As described in Ref. [6] (and references therein), the virtual non-factorizable corrections can be written in terms of a correction factor $\delta_{\text {nfact,DPA }}^{\text {virt }}$ to the lowest-order cross section. This factor is calculated in eeWW4f_DPA.f by the function DNONF with the possibility of choosing between different implementations (QNF $=1,2,3$ ), as described in Section 4.1.

The remaining part of the electroweak radiative corrections, i.e. the soft and collinear parts of virtual and real photon radiation and the hard bremsstrahlung contribution are calculated exactly, i.e. using off-shell kinematics and based on the full matrix elements to $\mathrm{e}^{+} \mathrm{e}^{-} \rightarrow 4 f$ and $\mathrm{e}^{+} \mathrm{e}^{-} \rightarrow 4 f \gamma($ SSIGEPEM5 $=5)$ of the tree-level mode. The matching of the virtual photon corrections calculated in DPA with the exact real photon radiation cannot be done unambiguously as far as the IR-finite terms are concerned. The definition of the virtual singular cross section is a matter of convention, since finite terms can be redistributed between singular and finite parts. This freedom, which is beyond DPA accuracy, is reflected by the different choices provided by RACOONWW in defining $\mathrm{d} \sigma_{\text {virt,finite,DPA }}^{\mathrm{e}^{+} \mathrm{e}^{-} \rightarrow \mathrm{WW}}$ $(\mathrm{QSOFT}=1,2,3$ and, for the subtraction branch, $\mathrm{SSUB}=1,2)$.

The singular part of the virtual corrections, $\mathrm{d} \sigma_{\text {virt,sing }}^{\mathrm{e}^{+} \mathrm{e}^{-} \rightarrow 4 f}$, factorizes into the lowest-order cross section and a simple correction factor. The correction factor of the part which is subtracted, $\mathrm{d} \sigma_{\text {virt,sing.DPA }}^{\mathrm{e}^{+} \mathrm{e}^{-} \rightarrow \text { WW } \rightarrow 4 f}$, is calculated in the slicing branch by the function DSOFT1 in eeWW4f_DPA.f for QSOFT $=1,2$ or by calling SOFTFAC in kern.f for QSOFT $=3$. In the subtraction branch, the part which is subtracted is calculated by the subroutine SUB4 in subtraction.f for QSOFT $=1,2,3$. The corresponding lowest-order cross section to $\mathrm{e}^{+} \mathrm{e}^{-} \rightarrow \mathrm{WW} \rightarrow 4 f$ is calculated in the subroutine CALCSME in eeWW4f_amps.f.

The soft and collinear singularities in the real part, $\mathrm{d} \sigma_{\text {sing }}^{\mathrm{e}^{+} \mathrm{e}^{-} \rightarrow 4 f \gamma}$, can be split off, and the integration over the singular regions of the photon phase space can be performed analytically. The result can be written as a convolution of a structure function with the lowest-order cross section $\mathrm{d} \sigma_{\text {Born }}^{\mathrm{e}^{+} \mathrm{e}^{-} \rightarrow 4 f}$. When adding $\mathrm{d} \sigma_{\text {virt,sing }}^{\mathrm{e}^{+} \mathrm{e}^{-} \rightarrow 4 f}$ and $\mathrm{d} \sigma_{\text {sing }}^{\mathrm{e}^{+} \mathrm{e}^{-} \rightarrow 4 f \gamma}$, all soft singularities and all collinear singularities associated with the final state cancel, and the resulting "singular" cross section,

$$
\mathrm{d} \sigma_{\text {virt+real,sing }}^{\mathrm{e}^{+} \mathrm{e}^{-} \rightarrow 4 f}=\mathrm{d} \sigma_{\text {virt,sing }}^{\mathrm{e}^{+} \mathrm{e}^{-} \rightarrow 4 f}+\mathrm{d} \sigma_{\text {sing }}^{\mathrm{e}^{+} \mathrm{e}^{-} \rightarrow 4 f \gamma},
$$

contains, apart from finite terms, only collinear singularities associated with the initial state, i.e. leading logarithms of the form $\ln \left(s / m_{\mathrm{e}}^{2}\right)$, at least for sufficiently inclusive observables.

Finally, we arrive at the master formula for the cross section:

$$
\mathrm{d} \sigma=\mathrm{d} \sigma_{\text {Born }}^{\mathrm{e}^{+} \mathrm{e}^{-} \rightarrow 4 f}+\mathrm{d} \sigma_{\text {virt,finite,DPA }}^{\mathrm{e}^{+} \mathrm{e}^{-} \rightarrow \mathrm{WW} \rightarrow 4 f}+\mathrm{d} \sigma_{\text {virt+real,sing }}^{\mathrm{e}^{+} \mathrm{e}^{-} \rightarrow 4 f}+\mathrm{d} \sigma_{\text {finite }}^{\mathrm{e}^{+} \mathrm{e}^{-} \rightarrow 4 f \gamma} .
$$

Since the contribution $\mathrm{d} \sigma_{\text {virt+real,sing }}^{\mathrm{e}^{+} \mathrm{e}^{-} \rightarrow 4 f}$ is not treated in DPA, the LL photonic corrections resulting from ISR are treated exactly in our approach. 
As a result, in the RC mode RACOONWW calculates several weights corresponding to processes with $4 f$ and $4 f \gamma$ kinematics. The cross sections $\mathrm{d} \sigma_{\text {Born }}^{\mathrm{e}^{+} \mathrm{e}^{-} \rightarrow 4 f}, \mathrm{~d} \sigma_{\text {virt,finite,DPA }}^{\mathrm{e}^{+} \mathrm{e}^{-} \rightarrow W W}$, $\mathrm{d} \sigma_{\text {virt+real }, \text { sing }}^{\mathrm{e}^{+} \mathrm{e}^{-} \rightarrow 4 f}$, and $\mathrm{d} \sigma_{\text {finite }}^{\mathrm{e}^{+} \mathrm{e}^{-} \rightarrow 4 f \gamma}$ of Eq. (36) are obtained from the corresponding $4 f$ weights and the $4 f \gamma$ weight, respectively.

\subsubsection{Higher-order leading-logarithmic (LL) corrections}

Higher-order LL corrections from ISR can be taken into account in all three modes by setting SISR $=1$ in the input file and are implemented in LL approximation via the structure-function approach (see Section 5 of Ref. [6]), i.e. by convoluting the tree-level or IBA cross section with the structure functions of Refs. $[9,18]$. The ISR structure functions depend on the QED splitting scale, $Q=$ SCALE, which is not fixed in LL approximation and has to be set to a typical momentum scale of the process. In the $\mathrm{RC}$ mode $\mathcal{O}(\alpha)$ corrections from ISR are calculated completely, and the $\mathcal{O}(\alpha)$ LL corrections are implicitly included. In order to avoid double counting of radiative corrections, the LL $\varnothing(\alpha)$ contributions from structure functions multiplied by the CC11 Born cross section are subtracted from the full $\varnothing(\alpha)$ corrections. Thus, for SRC $=1$ and SISR $=1$, the dependence on the QED scale, $Q$, only enters in $\varnothing\left(\alpha^{2}\right)$, i.e. at the two-loop level.

In the slicing branch of RACOONWW the ISR structure functions are calculated in kern.f by

$$
\begin{aligned}
& \text { FUNCTION } \operatorname{STRUCI}(\mathrm{XX}, \mathrm{DXX}, \mathrm{LLE}, \mathrm{BET}, \mathrm{ALP}) \\
& \mathrm{XX}, \mathrm{DXX} \quad: \text { random numbers, DXX }=1-\mathrm{XX}, \\
& \mathrm{LLE}, \mathrm{BET}, \mathrm{ALP}: \mathrm{BET}=\mathrm{ALP}(\mathrm{LLE}-1) / \pi, \mathrm{LLE}=2 \ln \left(\mathrm{SCALE} / m_{\mathrm{e}}\right), \mathrm{ALP}=\alpha(0) .
\end{aligned}
$$

In the subtraction branch they are obtained by calling

$$
\begin{array}{ll}
\text { SUBROUTINE } & \text { ISR (RANDOM, X1, X2, STFCT, WISR, SWITCH) } \\
\text { RANDOM(1:2) } & : \text { random numbers, } \\
\mathrm{X} 1, \mathrm{X} 2 & : \text { energy fraction of incoming particles (after ISR), } \\
\text { STFCT } & : \text { value of the structure functions only, } \\
\text { WISR } & : \text { total weight for generation of X1, X2 including structure functions, } \\
\text { SWITCH } & : \text { auxiliary flag (SWITCH }=0 \text { if an error occurs), }
\end{array}
$$

in subtraction.f. By default, SCALE is set to the CM energy, SCALE $=\sqrt{s}$, in the subroutine INITIALIZE in racoonww.f.

\subsubsection{Naive QCD corrections}

In all three modes of RACOONWW, the so-called naive QCD corrections can be included by setting QQCD $=2$ in the input file. QCD corrections enter the processes with hadronic final-states at two different places. On the one hand, the hadronic W-boson width receives 
a QCD correction, namely a factor $\left(1+\alpha_{\mathrm{s}} / \pi\right)[34,35]$, if the W-boson width is calculated $(\mathrm{QGW}=4)$. This affects the total W-boson width $\Gamma_{\mathrm{W}}$ in the $\mathrm{W}$ propagators. On the other hand, each hadronically decaying $\mathrm{W}$ boson receives a $\mathrm{QCD}$ correction to the $\mathrm{W} q \bar{q}^{\prime}$ vertex. If the full phase space for gluon emission is integrated over, this correction reduces to a multiplicative correction factor $\left(1+\alpha_{\mathrm{s}} / \pi\right)$ for each hadronically decaying $\mathrm{W}$ boson in $\varnothing\left(\alpha_{\mathrm{s}}\right)$. The application of this inclusive factor to distributions is usually called naive QCD correction. In the total cross section for W-pair production naive QCD-correction factors cancel against the corresponding factors in the W-boson width in the resonant W propagators.

\subsubsection{Anomalous triple and quartic gauge-boson couplings}

In RACOONWW anomalous triple gauge-boson couplings (ATGCs) to $\mathrm{e}^{+} \mathrm{e}^{-} \rightarrow 4 f$ and anomalous quartic gauge-boson couplings (AQGCs) to $\mathrm{e}^{+} \mathrm{e}^{-} \rightarrow 4 f \gamma$ have been implemented. More precisely, the non-standard couplings are introduced in the lowest-order matrix elements, which can be dressed by initial-state radiation, while the contributions from the remaining radiative corrections add linearly to the non-standard effects. The ATGC contributions are taken into account when SATGC $=1$ is set in the input file (see also Ref. [15]). The charged ATGCs are defined as in Ref. [36]. The conventions for the neutral ATGCs follow those of Refs. [37,38]. The ATGC contributions to the matrix elements are expressed in terms of five generic functions corresponding to the $\mathrm{ZVV}$ and $V W W$ couplings with $V=\gamma, \mathrm{Z}$, which have been implemented in the subroutines ADDMATGCVVZ and ADDMATGCVWW of ee4fa_amps.f, respectively. These ATGC contributions are added to the generic CC and NC helicity amplitudes in the subroutines M_NC and M_CC. When SATGC $=1$ is chosen the result depends on the ATGC parameters $\Delta g_{1}^{V}$, $\Delta \kappa^{V}, \lambda^{V}, \tilde{\kappa}^{V}, \tilde{\lambda}^{V}, g_{4,5}^{V}, f_{4,5}^{V}, h_{1,3}^{V}$ with $V=\gamma, \mathrm{Z}$, which are specified in the input file (see Section 4.1).

As described in detail in Ref. [8], AQGCs that involve at least one photon have been implemented in RACOONWW. They are taken into account when QAQGC $=1$ is set in the input file. Only genuine AQGCs are considered, i.e. all operators that contribute also to ATGCs, such as the quadrilinear part of the well-known operator $F^{\mu \nu} W_{\nu}^{+, \rho} W_{\rho \mu}^{-}$, are omitted. Imposing in addition custodial $\mathrm{SU}(2)_{\mathrm{c}}$ invariance [39] to keep the $\rho$ parameter close to 1 , only operators of dimension 6 or higher occur. Following Refs. [8,40,41,42,43] dimension-6 operators for genuine AQGCs are considered that respect local $\mathrm{U}(1)_{\mathrm{em}}$ invariance and global custodial SU(2) invariance. These symmetries reduce the set of such operators to a phenomenologically accessible basis. More general AQGCs were discussed in Ref. [44]. The AQGC contributions are expressed in terms of three generic functions which correspond to the $\gamma \gamma V V$ and $\gamma \mathrm{ZWW}$ couplings with $V=\mathrm{W}, \mathrm{Z}$, respectively. They are added to the generic Standard Model functions in M_CC and M_NC by calling the subroutines ADDMAAQGCAAWW, ADDMAAQGCAAZZ, and ADDMAAQGCAZWW in ee4fa_amps.f. When

$\mathrm{QAQGC}=1$, the result depends on the AQGC parameters $a_{0, c, n} / \Lambda^{2}$ and $\tilde{a}_{0, n} / \Lambda^{2}$ specified in the input file (see Section 4.1). 


\begin{tabular}{|c|c|c|c|c|c|c|c|}
\hline \multirow{4}{*}{$\begin{array}{l}\text { flag } \\
\text { SRC }\end{array}$} & \multirow{4}{*}{$\frac{\text { set in }}{\text { input file }}$} & \multirow{4}{*}{$\begin{array}{l}\text { switch for } \\
\text { tree-level, IBA, } \\
\text { and RC mode }\end{array}$} & \multicolumn{5}{|c|}{ possible values ( $*$ means not relevant) } \\
\hline & & & \multirow{2}{*}{\multicolumn{2}{|c|}{$\begin{array}{l}\mathrm{SRC}=0 \\
\text { tree-level }\end{array}$}} & \multirow{3}{*}{$\begin{array}{c}\mathrm{SRC}=2 \\
\mathrm{IBA} \\
4 f\end{array}$} & \multirow{3}{*}{$\begin{array}{c}\mathrm{SRC}=3 \\
\mathrm{IBA} \\
4 f \gamma\end{array}$} & \multirow{3}{*}{$\begin{array}{c}\mathrm{SRC}=1 \\
\mathrm{RC} \\
4 f, 4 f \gamma\end{array}$} \\
\hline & & & & & & & \\
\hline & & & $4 f$ & $4 f \gamma$ & & & \\
\hline SMC & input file & MC branch & 1,2 & 1,2 & 1,2 & 1,2 & $1-3$ \\
\hline SBORN4 & input file & tree-level $4 f$ & $1-3$ & 0 & 0 & 0 & $0-3$ \\
\hline SBORN5 & input file & tree-level $4 f \gamma$ & 0 & 1 & 0 & 1 & 1 \\
\hline SISR & input file & higher-order ISR & 0,1 & 0,1 & 1 & 1 & 0,1 \\
\hline SCOULTREE & input file & $\begin{array}{l}\text { Coulomb sing. for } \\
\mathrm{e}^{+} \mathrm{e}^{-} \rightarrow 4 f, 4 f \gamma\end{array}$ & 0,1 & $0-2$ & $*$ & $0-2$ & $*$ \\
\hline QNF & input file & $\begin{array}{l}\text { Coulomb sing. for } \\
\mathrm{e}^{+} \mathrm{e}^{-} \rightarrow 4 f \text { (RC) }\end{array}$ & $*$ & $*$ & * & $*$ & $1-3$ \\
\hline QREAL & input file & imaginary part & $*$ & $*$ & $*$ & $*$ & 0,1 \\
\hline QALP & input file & parameter scheme & $0-2$ & $0-2$ & 2 & 2 & $0-2$ \\
\hline QGW & input file & W-boson width & $0-4$ & $0-4$ & 0,2 & 0,2 & $0-4$ \\
\hline QPROP & input file & width approach & $0-4$ & $0-4$ & 1 & 1 & 1 \\
\hline SSIGEPEM4 & input file & sub-diagrams $(4 f)$ & $0-5$ & $*$ & 1 & $*$ & $0-5$ \\
\hline SSIGEPEM5 & input file & sub-diag. $(4 f \gamma)$ & $*$ & $0-5$ & $*$ & $0-5$ & 5 \\
\hline QQCD & input file & QCD RCs & 0,2 & 0,2 & 0,2 & 0,2 & 0,2 \\
\hline SQCDEPEM & input file & gluon-ex. backg. & $0-2$ & $0-2$ & $*$ & 0,1 & 0,1 \\
\hline SRECOMB & input file & photon recomb. & $*$ & $0-3$ & $*$ & $0-3$ & $0-3$ \\
\hline SCUTS & input file & separation cuts & $0-11$ & $0-11$ & $0-11$ & $0-11$ & $0-11$ \\
\hline SATGC & input file & ATGCs & 0,1 & $*$ & 0,1 & $*$ & 0,1 \\
\hline QAQGC & input file & AQGCs & $*$ & 0,1 & $*$ & 0,1 & 0 \\
\hline QFAST & racoonww.f & Legendre expans. & * & $*$ & $*$ & $*$ & 0,1 \\
\hline SON & racoonww.f & on-shell projection & $1-4$ & * & $*$ & $*$ & $1-4$ \\
\hline QSOFT & racoonww.f & photon DPA part & $*$ & $*$ & $*$ & $*$ & $1-3$ \\
\hline $\mathrm{OPT}$ & slicing.f & optimization & 0,1 & 0,1 & 0,1 & 0,1 & 0,1 \\
\hline SSUB & subtraction.f & subtr. functions & $*$ & $*$ & $*$ & $*$ & 1,2 \\
\hline
\end{tabular}

Table 3

The allowed choices for the options in the different modes of RACOONWW

\section{The usage of RACOONWW}

In the sequel we describe the input parameters and flags for the options of RACOONWW, starting with those that are set in the input file.A list of all possible values of the flags of RACOONWW discussed below is given in Table 3. Some more information on the options and details about the format in the input file can be found in the file README.

2 The flags SBORNG5, SSIGEPEMG5, SRECOMBG, and the parameters PRECOMBG are not listed here and are not discussed any further, since they only affect the QCD corrections beyond the naive approximation, which are not supported by RACOONWW yet. 


\subsection{Input and initialization of RACOONWW via the input file}

OUTPUTFILE: a character string that specifies the name of the output file.

ENERGY: the total CM energy in GeV $($ ENERGY $=\sqrt{s})$.

NEVENTSW: number of weighted events.

The number of weighted events must be at least NEVENTSW $=2 \times 10^{6}$ in the tree-level and IBA modes and NEVENTSW $=10^{7}$ in the $\mathrm{RC}$ mode to guarantee that the multi-channel integration yields reliable estimates for the $\mathrm{MC}$ integration error. When unweighted events are generated (NEVENTSUNW $>0$ ), NEVENTSW is the number of weighted events used to initialize the unweighting procedure; these weighted events are used to determine the maximal weights (see Section 4.4).

SMC : flag for the slicing and subtraction branches of RACOONWW:

$\mathrm{SMC}=1:$ chooses the slicing branch in its default setup,

SMC $=2$ : chooses the subtraction branch,

$\mathrm{SMC}=3:$ chooses a slightly modified version of the slicing branch, which has been introduced to minimize the number of negative weights in the $\mathrm{RC}$ mode.

In the RC mode, RACOONWW provides an unweighting procedure only for $\mathrm{SMC}=3$. More details can be found in Section 4.4.

SBORN4: flag for the tree-level $\mathrm{e}^{+} \mathrm{e}^{-} \rightarrow 4 f$ cross section:

SBORN4 $=0$ : the tree-level contribution to $\mathrm{e}^{+} \mathrm{e}^{-} \rightarrow 4 f$ is not included,

SBORN4 $=1$ : calculates the off-shell Born $\mathrm{e}^{+} \mathrm{e}^{-} \rightarrow 4 f$ cross section,

SBORN4 $=2$ : calculates the cross section to $\mathrm{e}^{+} \mathrm{e}^{-} \rightarrow \mathrm{WW} \rightarrow 4 f$ in DPA with phasespace for off-shell $\mathrm{W}$ bosons,

SBORN4 $=3$ : same as SBORN4 $=2$ but with phase-space for on-shell $\mathrm{W}$ bosons.

For SBORN4 $=1$ the selection of a subset of diagrams is governed by the flag SSIGEPEM4

(see Table 4). For SBORN4 = 2, 3 the Born cross section is based on the CC03 diagrams only. While for SBORN4 $=2$ the full phase space for off-shell $\mathrm{W}$ bosons is generated, the matrix element is calculated with on-shell momenta which result from a projection of the phase space with off-shell $\mathrm{W}$ bosons onto a phase space with on-shell $\mathrm{W}$ bosons. For SBORN4 $=3$ both the phase space and the matrix element squared are calculated with on-shell kinematics. In both cases the Z-boson width is set to zero. The flags SBORN4 $=2,3$ should only be used for checks and comparisons but not for the generation of physical results. Moreover, SBORN4 $=2,3$ is only a valid choice for $\mathrm{CC}$ processes or mixed processes with $\mathrm{SYM}=0,3$ (see Table 2 ).

SBORN5: flag for the tree-level cross section to $\mathrm{e}^{+} \mathrm{e}^{-} \rightarrow 4 f \gamma$ :

SBORN5 $=0$ : the tree-level contribution to $\mathrm{e}^{+} \mathrm{e}^{-} \rightarrow 4 f \gamma$ is not included, SBORN5 $=1$ : calculates the tree-level cross section to $\mathrm{e}^{+} \mathrm{e}^{-} \rightarrow 4 f \gamma$.

In the tree-level mode with SBORN5 $=1$, RACOONWW calculates the Born contribution to $\mathrm{e}^{+} \mathrm{e}^{-} \rightarrow 4 f \gamma$. In the $\mathrm{RC}$ mode $(\mathrm{SRC}=1)$ and in the IBA mode to $\mathrm{e}^{+} \mathrm{e}^{-} \rightarrow 4 f \gamma$ $(\mathrm{SRC}=3)$, RACOONWW always sets SBORN5 $=1$ in racoonww. $f$ independent of the 
choice in the input file. In the slicing branch $(\mathrm{SMC}=1,3)$, when radiative corrections to $\mathrm{e}^{+} \mathrm{e}^{-} \rightarrow \mathrm{WW} \rightarrow 4 f$ are calculated ( $\left.\mathrm{SRC}=1\right)$, SBORN5 $=1$ calculates the hardphoton bremsstrahlung contribution, i.e. photon radiation away from soft and collinear singularities.

SISR: flag for the inclusion of higher-order ISR:

SISR $=0$ : higher-order LL corrections from ISR are not included,

SISR $=1$ : higher-order LL corrections from ISR are included in the LL approximation up to order $\varnothing\left(\alpha^{3}\right)$ for $\mathrm{SMC}=1,2$ and up to order $\varnothing\left(\alpha^{2}\right)$ for $\mathrm{SMC}=3$.

Higher-order LL corrections from ISR are taken into account by convoluting the treelevel cross section with the structure functions of Refs. [9,18] (see also Section 3.2). In the RC mode the ISR beyond $\varnothing(\alpha)$ is switched on/off by the choice of SISR, i.e. $\varnothing(\alpha)$ ISR contributions are always included as part of the $\varnothing(\alpha)$ corrections even when $\mathrm{SISR}=0$. In the RC mode with SISR $=0$ the full $\varnothing(\alpha)$ ISR contribution is included for all diagrams of the CC11 process class. For SRC $=1$ and SISR $=1$ the LL contribution to the ISR is taken into account for the full four-fermion process, i.e. also for the background diagrams, depending on the choices for SSIGEPEM4. When ATGCs are considered $($ SATGC $=1)$, the complete ISR corrections in LL approximation $(\varnothing(\alpha)+$ higher-orders $)$ to the additional contributions involving anomalous couplings is also switched on/off by the choice of SISR. When calculating the IBA $(\mathrm{SRC}=2,3)$, or the tree-level processes $\mathrm{e}^{+} \mathrm{e}^{-} \rightarrow 4 f$ or $\mathrm{e}^{+} \mathrm{e}^{-} \rightarrow 4 f \gamma(\mathrm{SRC}=0, \operatorname{SISR}=1$, SBORN4 $=1$ or SBORN5 $=1)$, the full corrections from ISR are taken into account in LL approximation up to order $\varnothing\left(\alpha^{3}\right)$.

SRC: flag for choosing the tree-level, IBA, or RC mode:

$\mathrm{SRC}=0$ (tree-level mode) : calculation of the tree-level processes $\mathrm{e}^{+} \mathrm{e}^{-} \rightarrow 4 f$ $($ SBORN4 $=1)$ or $\mathrm{e}^{+} \mathrm{e}^{-} \rightarrow 4 f \gamma($ SBORN5 $=1)$,

$\mathrm{SRC}=1(\mathrm{RC}$ mode $) \quad: \varnothing(\alpha)$ radiative corrections to $\mathrm{e}^{+} \mathrm{e}^{-} \rightarrow \mathrm{WW} \rightarrow 4 f$ are included in DPA,

$\mathrm{SRC}=2$ (IBA mode) $\quad:$ IBA for $\mathrm{e}^{+} \mathrm{e}^{-} \rightarrow \mathrm{WW} \rightarrow 4 f$ is calculated for the CC03 class of diagrams,

$\mathrm{SRC}=3$ (IBA mode) $\quad:$ IBA for $\mathrm{e}^{+} \mathrm{e}^{-} \rightarrow 4 f \gamma$ is calculated based on the full matrix element.

SCOULTREE: flag for the inclusion of the Coulomb singularity in the tree-level and IBA modes:

SCOULTREE $=0$ : the Coulomb singularity to $\mathrm{e}^{+} \mathrm{e}^{-} \rightarrow 4 f$ and $\mathrm{e}^{+} \mathrm{e}^{-} \rightarrow 4 f \gamma$ is not included,

SCOULTREE $=1$ : the off-shell Coulomb singularity to $\mathrm{e}^{+} \mathrm{e}^{-} \rightarrow 4 f$ in the tree-level mode and to $\mathrm{e}^{+} \mathrm{e}^{-} \rightarrow 4 f \gamma$ in the tree-level and IBA modes is included,

$\operatorname{SCOULTREE}=2$ : the off-shell Coulomb singularity to $\mathrm{e}^{+} \mathrm{e}^{-} \rightarrow 4 f \gamma$ in the tree-level and IBA modes is included.

For SCOULTREE $=1$ the Coulomb singularity is calculated with invariant masses derived from the four-momenta of the final-state fermions only. For SCOULTREE $=2$, which is only a valid choice for the processes $\mathrm{e}^{+} \mathrm{e}^{-} \rightarrow 4 f \gamma$, the Coulomb singularity is calcu- 
lated with the invariant masses of those $\mathrm{W}^{+}$and $\mathrm{W}^{-}$bosons which are most resonant. Note that the invariant masses of the $\mathrm{W}$ bosons may or may not include the photon momentum depending whether the photon is emitted from the initial or final state (see Section 3.2). The choices SCOULTREE $>0$ are only valid for WW mediated processes, i.e. for $\mathrm{CC}$ processes and mixed processes with $\mathrm{SYM}=0,3$.

QNF : flag for the inclusion for the Coulomb singularity in the RC mode:

$\mathrm{QNF}=1:$ DPA Coulomb singularity,

$\mathrm{QNF}=2:$ off-shell Coulomb singularity with DPA Born cross section,

QNF $=3$ : off-shell Coulomb singularity with (off-shell) CC03 Born cross section.

This flag is only effective in the RC mode. According to the choice of QNF, different implementations of the Coulomb singularity are included in the virtual non-factorizable corrections (the real non-factorizable corrections are implicitly contained in the full matrix-element calculation of $\mathrm{e}^{+} \mathrm{e}^{-} \rightarrow 4 f \gamma$ ). The off-shell Coulomb singularity is implemented as defined in Ref. [17]. In Ref. [6] different versions of the DPA have been investigated by modifying the implementation of the DPA. Different treatments of the Coulomb singularity, QNF $=2$ ("def") and QNF $=1$ ("Coul"), are one of the considered options. The recommended setting is QNF $=3$.

QREAL: flag for the inclusion of the imaginary parts of the virtual electroweak corrections: QREAL $=0$ : imaginary parts of the one-loop corrections are included,

QREAL $=1$ : imaginary parts of the one-loop corrections are not included.

This flag is only effective in the RC mode. The imaginary parts of the one-loop corrections contribute only significantly to distributions that depend on the azimuthal decay angle of one of the $\mathrm{W}$ bosons. If such distributions are considered, QREAL has to be set to QREAL $=0$ (imaginary parts are not neglected).

QALP: flag to specify the input-parameter scheme:

QALP $=0: \alpha(0)$ scheme,

QALP $=1: \alpha\left(M_{Z}\right)$ scheme,

$\mathrm{QALP}=2: G_{\mu}$ scheme.

Independent of the choice of the input-parameter scheme, the electroweak corrections are always calculated by using the fine structure constant $\alpha(0)$, i.e. the relative (electroweak) corrections $\left(\mathrm{d} \sigma-\mathrm{d} \sigma_{\text {Born }}\right) / \mathrm{d} \sigma_{\text {Born }}$ are always proportional to $\alpha(0)$. Not all input parameters defined in the subroutine PARAMETER in public.f are used in the different input-parameter schemes, e.g. ALPHAZ is only relevant for QALP $=1$, GF only for $\mathrm{QALP}=2$. While the small fermion masses have to be non-zero for all input-parameter schemes, their actual values matter only if QALP $=0$, except for the electron mass that is used in the structure functions.

QGW: flag for the W-boson width: 
QGW $=0$ : the total W-boson width is an input parameter, set in the subroutine PARAMETER in public.f,

$\mathrm{QGW}=1$ : the tree-level $\mathrm{W}$-boson width is calculated, $\mathrm{QGW}=2$ : the IBA W-boson width is calculated,

$\mathrm{QGW}=3$ : the one-loop $\mathrm{W}$-boson width is calculated without $\varnothing\left(\alpha_{s}\right)$ corrections, $\mathrm{QGW}=4$ : the one-loop $\mathrm{W}$-boson width is calculated with $\varnothing\left(\alpha_{s}\right)$ corrections.

When the one-loop W-boson width is calculated (QGW $=3,4$ and $\mathrm{SRC}=0,1$ ), the full electroweak $\varnothing(\alpha)$ corrections in the chosen input-parameter scheme (controlled by the flag QALP) are included. When $\mathrm{QGW}=2$, the W-boson width is calculated in IBA, i.e. the tree-level W-boson width in the $G_{\mu}$ scheme (QALP $=2$ ) including $\varnothing\left(\alpha_{s}\right)$ corrections. The only allowed options for $\mathrm{SRC}=2,3$ are $\mathrm{QGW}=0,2$. In racoonww. $\mathrm{f}$ the partial decay widths for the decays into leptons and quarks are also calculated using the same options as for the calculation of the amplitude. In the output file, RACOONWW provides the "effective" branching ratios: leptonic width/total width, hadronic width/total width, and (leptonic + hadronic width)/total width. The widths in the numerators are calculated with the same options as the DPA matrix element for $\mathrm{e}^{+} \mathrm{e}^{-} \rightarrow \mathrm{WW} \rightarrow 4 f$. Since the total $\mathrm{W}$-boson width and the partial widths are not necessarily calculated with the same options, the total branching ratio can be different from one. The effective branching ratios are not used in RACOONWW and are only provided for information.

QPROP: flag for choosing a width scheme:

$\mathrm{QPROP}=0$ : gauge-boson widths are set to zero,

$\mathrm{QPROP}=1$ : constant width is used in all propagators,

$\mathrm{QPROP}=2:$ constant width is used only in time-like propagators,

QPROP $=3$ : running width is used (no width in space-like propagators),

$\mathrm{QPROP}=4$ : constant width is used in all propagators and the weak mixing angle is calculated from the complex W- and Z-boson masses.

For $\mathrm{SRC} \neq 0, \mathrm{QPROP}=1$ is automatically chosen in the subroutine INITIALIZE in racoonww.f.

SSIGEPEM4: chooses a subset of diagrams for the tree-level processes $\mathrm{e}^{+} \mathrm{e}^{-} \rightarrow 4 f$ (see Table 4).

SSIGEPEM5: chooses a subset of diagrams for the tree-level and IBA processes $\mathrm{e}^{+} \mathrm{e}^{-} \rightarrow 4 f \gamma$ (see Table 4).

QQCD: flag for the inclusion of QCD corrections:

$\mathrm{QQCD}=0:$ QCD corrections are not included,

$\mathrm{QQCD}=2$ : naive QCD corrections are included.

In RACOONWW only so-called naive QCD corrections can be included yet, i.e. for each hadronically decaying $\mathrm{W}$ boson RACOONWW applies a global factor $\left(1+\alpha_{s} / \pi\right)$ to the differential cross sections.

FNAME $(3: 6)$ : particle names for the specification of the final-state fermions: 


\begin{tabular}{|c|l|}
\hline $\begin{array}{c}\text { SSIGEPEM4 } \\
\text { SSIGEPEM5 }\end{array}$ & subset of diagrams included \\
\hline 0 & all electroweak diagrams \\
1 & only electroweak WW signal diagrams (CC03) \\
2 & only electroweak $\mathrm{ZZ} \mathrm{signal} \mathrm{diagrams} \mathrm{(NC02)}$ \\
3 & only electroweak $\mathrm{ZZ}, \mathrm{Z} \gamma$, and $\gamma \gamma$ signal diagrams \\
4 & only electroweak $\mathrm{WW}, \mathrm{ZZ}, \mathrm{Z} \gamma$, and $\gamma \gamma$ signal diagrams \\
5 & only diagrams corresponding to the CC11 process class \\
\hline
\end{tabular}

Table 4

Possible choices for subsets of Feynman diagrams as governed by SSIGEPEM4 and SSIGEPEM5

FNAME $=u, d, s, c, t, b, e, m u$, tau, nu_e, nu_mu, nu_tau.

When specifying the final-state particles the order of Table 2 must be respected.

PP , PM: degrees of positron- and electron-beam polarization; for unpolarized beams choose $\mathrm{PP}=\mathrm{PM}=0$.

SRECOMB: flag for the photon-recombination scheme:

$\mathrm{SRECOMB}=0:$ no recombination is performed,

$\mathrm{SRECOMB}=1: \mathrm{TH}$ recombination scheme is applied (see also Ref. [6]),

$\mathrm{SRECOMB}=2:$ EXP recombination scheme is applied (see also Ref. [6]),

$\mathrm{SRECOMB}=3: \mathrm{TH}$ recombination scheme is applied including photon recombination also with the beams.

When SRECOMB $=1,2,3$ the parameters $\operatorname{PRECOMB}(1: 4)$ must be specified in the input file (see below). For SRECOMB $=3$ no separation cuts are required and $\operatorname{PRECOMB}(1)=0$ can be chosen. Note that if the unweighting procedure is used, the recombination procedure and separation cuts influence the resulting unweighted events.

$\operatorname{PRECOMB}(1: 4)$ : recombination parameters:

PRECOMB (1): separation cut on angle between beam and photon in degrees if $\operatorname{SRECOMB}=1,2,3$,

$\operatorname{PRECOMB}(2)$ : recombination cut for photon energy in $\mathrm{GeV}$ if $\operatorname{SRECOMB}=1,2,3$,

$\operatorname{PRECOMB}(3)$ : invariant-mass recombination cut in $\mathrm{GeV}$ if $\operatorname{SRECOMB}=1,3$ or angular recombination cut for leptons in degrees if $\operatorname{SRECOMB}=2$,

$\operatorname{PRECOMB}(4)$ : angular recombination cut for quarks in degrees if SRECOMB $=2$.

The recombination scheme is defined in the subroutine RECOMBINATION in public.f. The recombination scheme for $\operatorname{SRECOMB}=1$ is described in detail in Ref. [6] for the parameters $\operatorname{PRECOMB}(1)=5, \operatorname{PRECOMB}(2)=1$, and $\operatorname{PRECOMB}(3)=5$ or 25 , called "bare" or "calo" cuts, respectively. For all three photon recombination schemes (SRECOMB $=$ $1,2,3)$, the photon momentum is set to zero if the angle between the photon and the beam is smaller than PRECOMB(1). This cut is applied at the very beginning before photon recombination is performed.

For SRECOMB $=1$, the minimal invariant mass of the photon and any charged finalstate fermion, $M(\gamma$, FCOMB $)$, is determined, and the photon is recombined with the 
final-state fermion FCOMB if $M(\gamma$, FCOMB $)<\operatorname{PRECOMB}(3)$ or the photon energy $E_{\gamma}<$ PRECOMB(2). Explicitly, this means that the photon momentum is added to the momentum of the fermion FCOMB, and the photon is discarded.

For $\operatorname{SRECOMB}=2$, the minimal angle between the photon and any charged final-state fermion, $\theta(\gamma$, FCOMB $)$, is determined, and the photon is recombined with the fermion FCOMB if $\theta\left(\gamma, l^{ \pm}\right)<\operatorname{PRECOMB}(3)$ in case $l^{ \pm}$is charged lepton of the final state or if $\theta(\gamma, q)<\operatorname{PRECOMB}(4)$ in case $q$ is a final-state quark, or if the photon energy $E_{\gamma}<$ $\operatorname{PRECOMB}(2)$.

For SRECOMB $=3$ the minimal invariant mass of the photon and any charged fermion (including the incoming electron and positron), $M(\gamma, \mathrm{FCOMB})$, is determined, and the photon is recombined with the fermion FCOMB if $M(\gamma, f)<\operatorname{PRECOMB}(3)$, where $f$ is one of the initial- or final-state charged fermions, or if $E_{\gamma}<\operatorname{PRECOMB}(2)$. In case FCOMB is a beam electron or positron, the photon momentum is set to zero.

SQCDEPEM: flag for the inclusion of gluon-exchange diagrams in the tree-level mode:

SQCDEPEM $=0$ : gluon-exchange background diagrams are not included,

SQCDEPEM $=1$ : gluon-exchange background diagrams are included,

SQCDEPEM $=2$ : only gluon-exchange background diagrams are included.

The choice of SQCDEPEM only affects the tree-level cross sections to $\mathrm{e}^{+} \mathrm{e}^{-} \rightarrow 4 f$ and $\mathrm{e}^{+} \mathrm{e}^{-} \rightarrow 4 f \gamma$, and for SISR $=1$ also the LL corrections from ISR. This QCD contribution then consists of gluon-exchange background diagrams to the tree-level processes $\mathrm{e}^{+} \mathrm{e}^{-} \rightarrow$ $4 f$ and $\mathrm{e}^{+} \mathrm{e}^{-} \rightarrow 4 f \gamma$ as described in Ref. [4]. In the RC mode gluon-exchange diagrams can be taken into account only in the tree-level matrix element to $\mathrm{e}^{+} \mathrm{e}^{-} \rightarrow 4 f$.

SATGC: flag for the inclusion of anomalous triple gauge-boson couplings (ATGCs):

SATGC $=0:$ ATGCs are not included,

SATGC $=1:$ ATGCs are included.

If $\operatorname{SATGC}=1$, the parameters GTGC $(1: 11,1: 2)$ must be specified in the input file. They are stored in the common block ATGC and correspond to the ATGCs $\Delta g_{1}^{V}, \Delta \kappa^{V}, \lambda^{V}$, $\tilde{\kappa}^{V}, \tilde{\lambda}^{V}, g_{4,5}^{V}, f_{4,5}^{V}, h_{1,3}^{V}$ with $V=\gamma(\equiv \mathrm{A})$, Z. The ATGCs affect only the tree-level cross section for $\mathrm{e}^{+} \mathrm{e}^{-} \rightarrow 4 f$, and for SISR $=1$ also the LL corrections from ISR are applied to the tree-level cross sections involving ATGCs.

QAQGC: flag for the inclusion of anomalous quartic gauge-boson couplings (AQGCs):

QAQGC $=0:$ AQGCs are not included,

QAQGC $=1:$ AQGCs are included.

If $\mathrm{QAQGC}=1$, the parameters ALAM2 $(0: 4)$ must be specified in the input file. They are stored in the common block AQGC and correspond to the AQGCs $a_{0, c, n} / \Lambda^{2}$ and $\tilde{a}_{0, n} / \Lambda^{2}$. The inclusion of AQGCs is not supported in the RC mode and only affects the results obtained in the tree-level mode $(\mathrm{SRC}=0, \mathrm{SBORN5}=1)$ and the IBA mode $(\mathrm{SRC}=3)$ for $\mathrm{e}^{+} \mathrm{e}^{-} \rightarrow 4 f \gamma$.

SCUTS: flag for choosing the separation cuts: 
SCUTS $=0 \quad:$ no separation cuts are applied,

SCUTS $=1 \quad:$ ADLO cuts are applied as defined in racoonww.f and in Ref. [10],

SCUTS $=2 \quad:$ LC cuts are applied as defined in racoonww.f,

SCUTS $=10:$ a minimal set of cuts must be specified in the input file,

SCUTS $=11$ : cuts on the energies of all final-state particles, on all angles, and on invariant masses must be specified in the input file.

All energies and invariant masses are measured in GeV and all angles in degrees. Nonstandard cuts, i.e. cuts which are not covered by the choices of SCUTS that RACOONWW provides, can be included at the end of the subroutine CUT in public.f.

\subsection{Options not provided in the input file}

RACOONWW has a few more options that are not provided in the input file. Changing those options is only advisable if the user is familiar with the details of the calculation as described in Ref. [6]. If they are common to both the slicing and subtraction branches, RACOONWW sets them to their default values in subroutine INITIALIZE in racoonww. $f$ (after "setting options"). Special options for the slicing and subtraction branches are set in the subroutines INITSLICING and INITSUBTRACTION, respectively.

The following flags are set in subroutine INITIALIZE in racoonww.f:

SON : flag for the projection of the phase space of the final-state particles to the one with on-shell W bosons. RACOONWW provides four different versions for the projection of off-shell momenta to on-shell momenta:

SON $=0$ : directions of momenta of $\mathrm{W}^{+}, f_{3}$, and $f_{5}$ are fixed (default),

$\mathrm{SON}=1$ : directions of momenta of $\mathrm{W}^{+}, \bar{f}_{4}$, and $f_{5}$ are fixed,

SON $=2:$ directions of momenta of $\mathrm{W}^{+}, f_{3}$, and $\bar{f}_{6}$ are fixed,

SON $=3$ : directions of momenta of $\mathrm{W}^{+}, \bar{f}_{4}$, and $\bar{f}_{6}$ are fixed

for the process $\mathrm{e}^{+} \mathrm{e}^{-} \rightarrow f_{3} \bar{f}_{4} f_{5} \bar{f}_{6}$ with $\mathrm{W}^{+} \rightarrow f_{3} \bar{f}_{4}$ and $\mathrm{W}^{-} \rightarrow f_{5} \bar{f}_{6}$.

Since the projections are performed in the CM frame of the incoming $\mathrm{e}^{+} \mathrm{e}^{-}$, the direction of the $\mathrm{W}^{-}$is opposite to the direction of the $\mathrm{W}^{+}$. The projection is done in the subroutine OFTOON in eeWW4f_DPA.f. In Ref. [6] the accuracy of the DPA has been investigated by modifying the implementation of the DPA and comparing the results. The comparison of the on-shell projections, SON $=1$ ("def") and SON $=2$ ("proj"), is one of the considered options.

QFAST: flag for the evaluation of the virtual corrections in DPA:

$\mathrm{QFAST}=0:$ form factors for virtual corrections from full formulas (slow),

$\mathrm{QFAST}=1$ : evaluation from expansion into Legendre polynomials (fast); this is the default value of QFAST.

Details of this approach can be found in Section 3.2.3 and in Ref. [6].

QSOFT: flag for the definition of the DPA part of radiative corrections. Terms that are 
subtracted from the virtual corrections in DPA are fixed by QSOFT $=1$ : endpoint part of Ref. [19] as given in Eq. (4.29) of Ref. [6] (default), QSOFT $=2$ : only logarithms as defined in Eq. (4.2.53) of Ref. [20], QSOFT $=3$ : YFS factor for virtual photons as defined in Eq. (4.31) of Ref. [6]. Note that results obtained with different choices for QSOFT differ only by terms that are beyond DPA accuracy. In Ref. [6] the accuracy of the DPA has been investigated by modifying the implementation of the DPA and comparing the results. The definition of the finite virtual corrections, QSOFT $=1$ ("def") and QSOFT $=3$ ("eik"), is one of the considered options.

RACOONWW provides the possibility to use PAW/HBOOK $[45,46]$ for histogram filling and plotting. The usage of $\mathrm{PAW} / \mathrm{HBOOK}$ is only allowed when generating weighted events (NEVENTSUNW=0). This option can be activated as follows:

- Set $\mathrm{SPAW}=1$ in the subroutine INITIALIZE in racoonww.f.

- Uncomment all lines in the if-environment starting with "if(spaw.eq.1..." in the subroutine INITIALIZE.

- Edit the makefile and add pawgraphs.o to the list of the objects OBJS. When using HBOOK/PAW, CERNLIB must be linked in the makefile.

In pawgraphs.f the histograms are defined in the subroutine SETUP_GRAPHS and filled in the subroutine GRAPHS, which are called in kern.f. This is the place where the user can add and modify histograms.

In the following we describe some branch-specific options of RACOONWW.

\subsubsection{Options specific for the slicing branch}

All the flags and parameters specific for the slicing branch are set in the subroutine INITSLICING in slicing.f:

OPT : flag for the optimization of the a priori weights:

$\mathrm{OPT}=0:$ no optimization,

$\mathrm{OPT}=1$ : self-optimization of the a priori weights (default).

In the slicing branch the self-optimization of the a priori weights is performed when $\mathrm{OPT}=1$ in slicing.f. The number of events used for the optimization of the a priori weights and how often the optimization is performed can be separately specified for the calculation of the $4 f$ weights (NOPT, IOPT) and the $4 f \gamma$ weights (NOPTG, IOPTG). The default setting is NOPT $=10^{6}$, IOPT $=10$, and NOPTG $=25 \times 10^{6}$, IOPTG $=1$. The flag

OPT and the parameters enter in function KERN in kern.f. The a priori weights are optimized as long as the number of generated events is smaller than NOPT or NOPTG. For the $\mathrm{e}^{+} \mathrm{e}^{-} \rightarrow 4 f$ part the a priori weights are optimized after $5 \times 10^{3}, 10 \times 10^{3}, 15 \times 10^{3}$, $25 \times 10^{3}$ generated events and when the number of events, NEVENTS, is a multiple of 
NOPT/IOPT as long as NEVENTS $\leq$ NOPT. For the $\mathrm{e}^{+} \mathrm{e}^{-} \rightarrow 4 f \gamma$ part the a priori weights are optimized after $5 \times 10^{5}, 15 \times 10^{5}, 30 \times 10^{5}$ generated events and when NEVENTS is a multiple of NOPTG/IOPTG as long as NEVENTS $\leq$ NOPTG.

\subsubsection{Options specific for the subtraction branch}

The flags and parameters specific for the subtraction branch are set in the subroutine INITSUBTRACTION in subtraction.f:

SSUB: flag for the definition of the subtraction functions:

$\operatorname{SSUB}=1:$ subtraction terms as defined in Ref. [19],

SSUB $=2:$ subtraction terms as defined in Ref. [20].

The results obtained by the two subtraction variants differ only by terms that are beyond the DPA accuracy. More details can be found in Ref. [6].

NOPT: parameters for the adaptive optimization.

NOPT defines the numbers of events after which the adaptive optimization is performed, i.e. after which event the a priori weights are recalculated. The optimization steps are given by $\operatorname{NOPT}(\mathrm{I}, \mathrm{NG}) \times$ number of channels, where $\mathrm{NG}=1,2,3$ denotes the phase-space generators discussed in Section 3.1.5. The adaptive optimization stops after the $i$-th optimization step if $\operatorname{NOPT}(\mathrm{I}+1, \mathrm{NG})=0$. The default settings are $\mathrm{NOPT}(\mathrm{I}, \mathrm{NG})=\mathrm{I} \times 100$ for $\mathrm{I}=1, \ldots, 8$ and zero otherwise.

ALPHAMIN : the a priori weights have a minimal value which is fixed by setting the parameter ALPHAMIN.

\subsection{The output of RACOONWW}

The output consists of an output file and 27 (or more, if the user added histograms) data files, which contain the data for the distributions. The name of the output file is set in the input file and stored in the variable OUTPUTFILE. For SPAW $=1$ there is also a PAW file pawplot.paw with the histograms generated with PAW/HBOOK $[45,46]$. If IOUT $\neq 0$ is set in the subroutine INITIALIZE in racoonww.f, a file optimization.info will be generated. This file contains information about the kinematical channels used in the multi-channel integration and about the optimization of the a priori weights as described in Section 3.1. By default IOUT $=0$ is set.

The event information is stored in the common block EVENT (see Section 2). The weights of the slicing and subtraction branches are described in Table 5 for SMC $=1$, in Table 6 for $\mathrm{SMC}=2$, and in Table 7 for $\mathrm{SMC}=3$. 


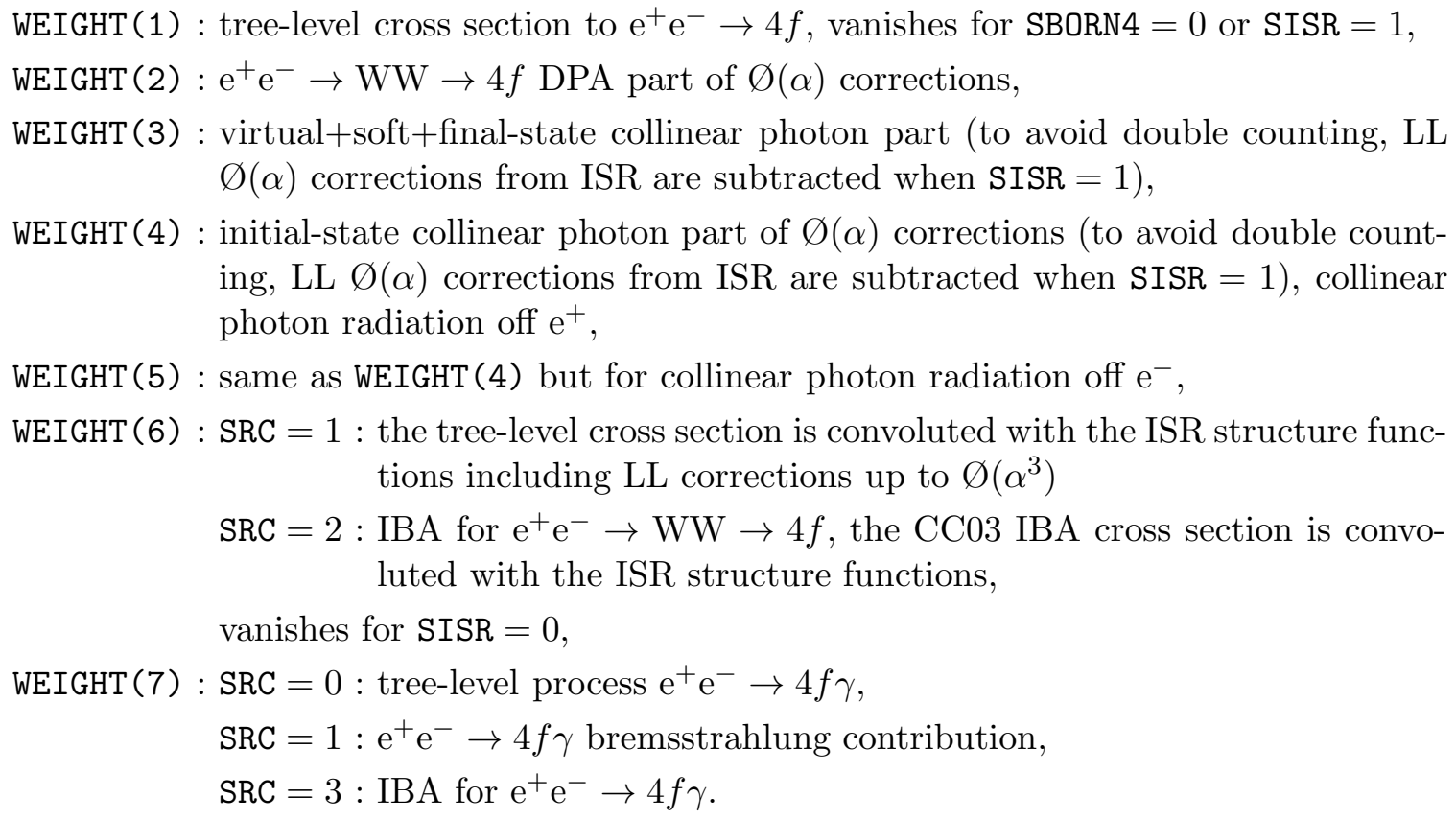

Table 5

Description of the weights WEIGHT (1:7) of the slicing branch $(\mathrm{SMC}=1)$

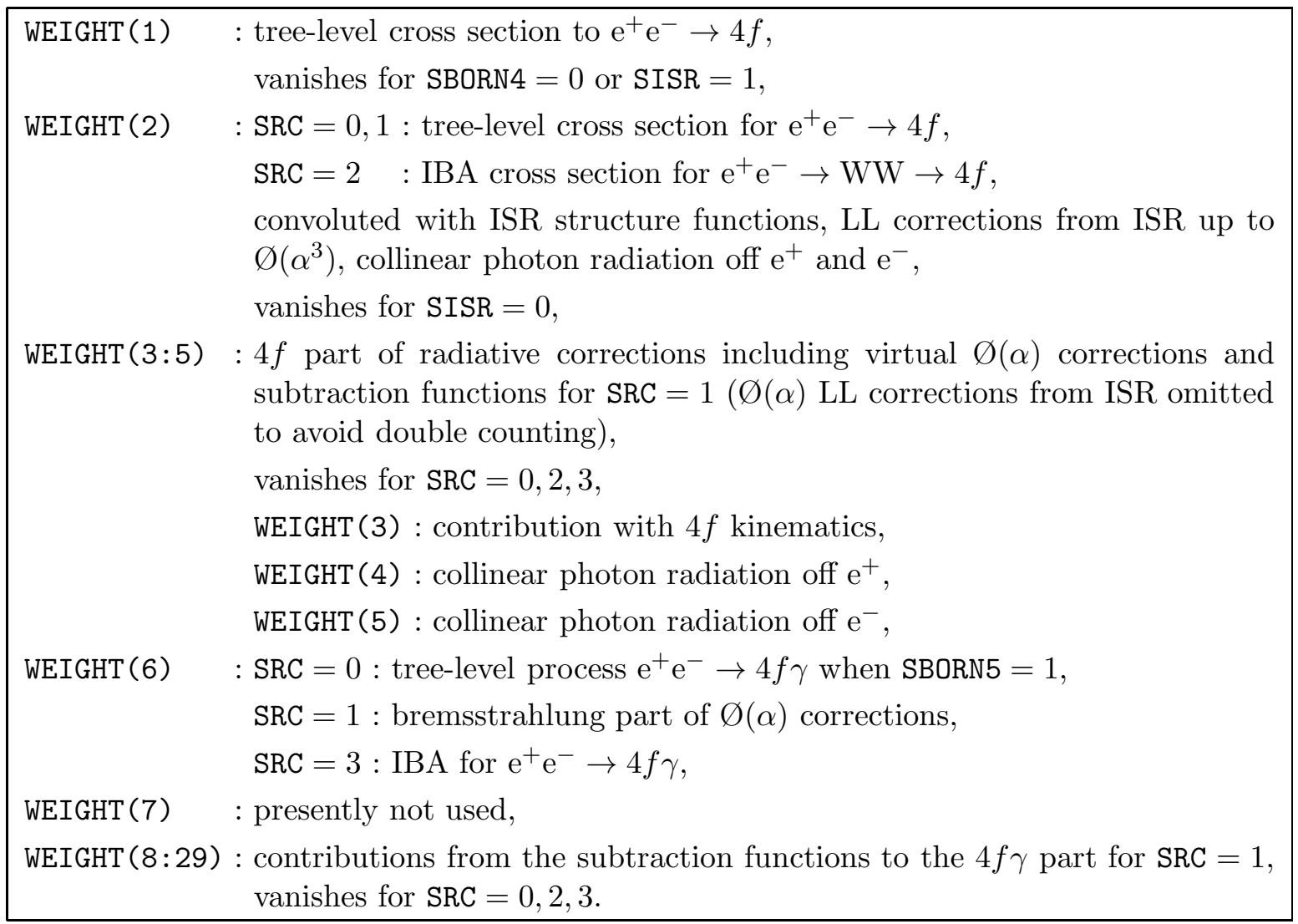

Table 6

Description of the weights WEIGHT (1:29) of the subtraction branch $($ SMC $=2)$ 


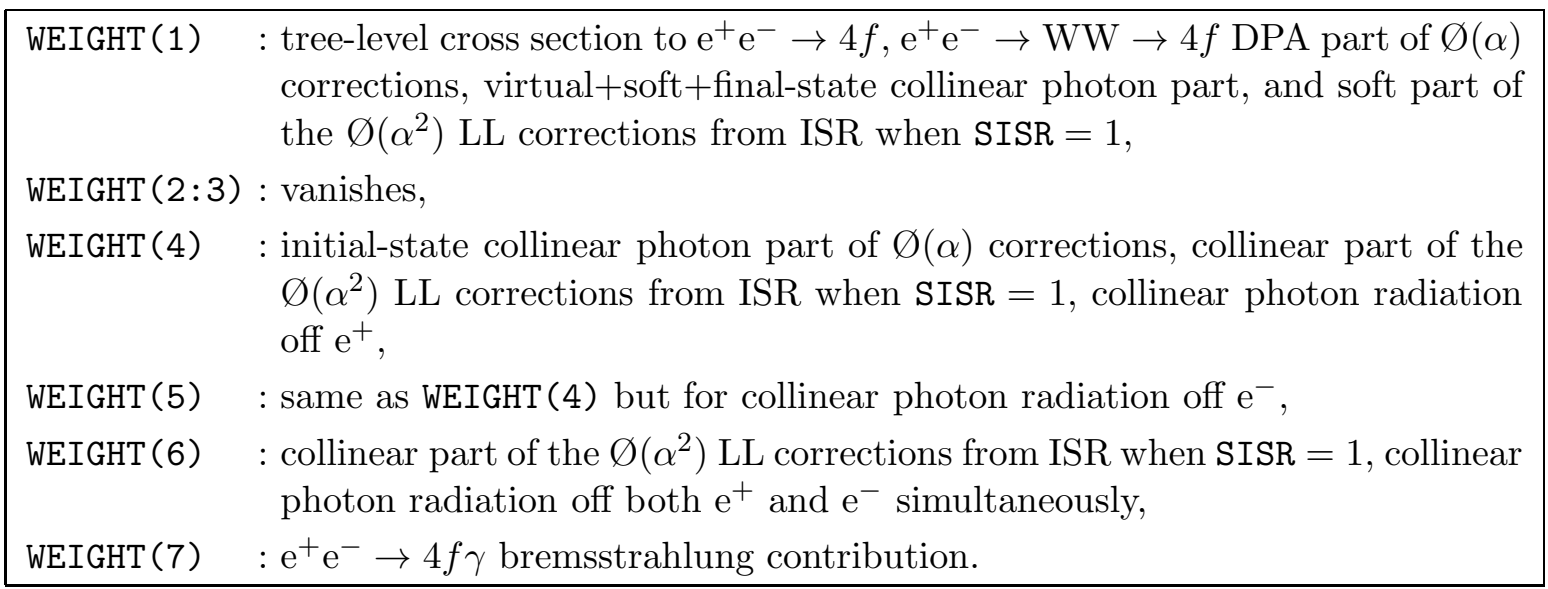

Table 7

Description of the weights WEIGHT (1:7) of the slicing branch $(\mathrm{SMC}=3)$. This branch is only supported in the $\mathrm{RC}$ mode $(\mathrm{SRC}=1)$

\subsubsection{The output file of RACOONWW}

In the output file RACOONWW provides the following information:

- the input parameters, the effective branching ratios, and the choices for the options,

- the total cross section together with the maximal values of the weights (in the subtraction branch also the corresponding event numbers and channels are given), and

- the total cross sections for the subcontributions (tree-level $4 f$ cross section, $2 \rightarrow 4$ part of the corrections, ISR up to $\varnothing\left(\alpha^{3}\right)$, and $2 \rightarrow 5$ part of the corrections) together with the maximal values of the weights.

Finally, the number of rejected events is given. Events are discarded owing to the applied separation cuts and in case of the slicing branch also owing to the imposed technical cuts. RACOONWW provides the number of rejected events in the calculation of each weight separately.

\subsubsection{The data files for distributions}

The data for the distributions are written in files dat.01,..,dat.27. The histograms are defined in the subroutine SETTINGS in public.f as described in Table 8. The file dat. 01 contains the total cross section for test purposes. Note that the momenta of the initial-state particles after ISR, $\mathrm{P}(1: 2, \mathrm{~J}, \mathrm{~K})$, do not correspond to physically measurable quantities and, thus, must not be used for defining histograms.

The data files contain the observable (column 1), the differential cross section for the chosen setup (column 2) and the corresponding lowest-order cross section of the tree-level process $\mathrm{e}^{+} \mathrm{e}^{-} \rightarrow 4 f$ (column 4 ). The statistical errors are provided in columns 3 and 5 , respectively. These are calculated as described in Eq. (15) and below. The histograms are normalized such that the total cross section is obtained as $\sigma=\sum_{\text {bins }}$ bin-height $\times$ bin-width. The angles of the histograms dat.21 and dat.22 are defined in Eqs. (7.4) and (7.3) of 


\begin{tabular}{|c|c|}
\hline data file & distribution in the \\
\hline dat.02 & $\begin{array}{l}\text { invariant mass of } f_{3} \text { and } \bar{f}_{4},[75: 85] \mathrm{GeV} \text { (in case of CC processes this corresponds } \\
\text { to the } \mathrm{W}^{+} \text {invariant mass), }\end{array}$ \\
\hline dat.03 & $\begin{array}{l}\text { invariant mass of } f_{5} \text { and } \bar{f}_{6},[75: 85] \mathrm{GeV} \text { (in case of } \mathrm{CC} \text { processes this corresponds } \\
\text { to the } \mathrm{W}^{-} \text {invariant mass), }\end{array}$ \\
\hline dat. 04 & cosine of the angle between $V_{1}$ and $\bar{f}_{1}=\mathrm{e}^{+},[-1: 1]$ \\
\hline dat. 05 & cosine of the angle between $V_{2}$ and $f_{2}=\mathrm{e}^{-},[-1: 1]$, \\
\hline dat.06 & cosine of the angle between $V_{1}$ and $f_{3},[-1: 1]$, \\
\hline dat. 07 & cosine of the angle between $V_{1}$ and $\bar{f}_{4},[-1: 1]$ \\
\hline dat. 08 & cosine of the angle between $V_{2}$ and $f_{5},[-1: 1]$ \\
\hline dat. 09 & cosine of the angle between $V_{2}$ and $\bar{f}_{6},[-1: 1]$, \\
\hline dat. 10 & energy of the photon, $[0: 50] \mathrm{GeV}$ ( $4 f$ weights are added to the first bin), \\
\hline dat. 11 & $\begin{array}{l}\text { cosine of the angle between } \gamma \text { and } \bar{f}_{1}=\mathrm{e}^{+},[-1: 1] \text { ( } 4 f \text { weights are added to the } \\
\text { last bin), }\end{array}$ \\
\hline dat.12 & $\begin{array}{l}\text { minimal angle between } \gamma \text { and charged final-state fermions, }[0: 180] \text { degrees }(4 f \\
\text { weights are added to the first bin), }\end{array}$ \\
\hline dat.13 & energy of $f_{3},[0: \sqrt{s} / 2] \mathrm{GeV}$, \\
\hline dat. 14 & energy of $\bar{f}_{4},[0: \sqrt{s} / 2] \mathrm{GeV}$, \\
\hline dat. 15 & energy of $f_{5},[0: \sqrt{s} / 2] \mathrm{GeV}$ \\
\hline dat. 16 & energy of $\bar{f}_{6},[0: \sqrt{s} / 2] \mathrm{GeV}$ \\
\hline dat. 17 & cosine of the angle between $V_{1}$ and $f_{3}$ in the rest frame of $V_{1},[-1: 1]$, \\
\hline dat. 18 & cosine of the angle between $V_{1}$ and $\bar{f}_{4}$ in the rest frame of $V_{1},[-1: 1]$, \\
\hline dat. 19 & cosine of the angle between $V_{2}$ and $f_{5}$ in the rest frame of $V_{2},[-1: 1]$, \\
\hline dat. 20 & cosine of the angle between $V_{2}$ and $\bar{f}_{6}$ in the rest frame of $V_{2},[-1: 1]$, \\
\hline dat. 21 & angle between decay planes of $f_{3}, \bar{f}_{4}$ and $f_{5}, \bar{f}_{6},[0: 360]$ degrees, \\
\hline dat. 22 & angle between decay planes of $V_{1}, \bar{f}_{1}=\mathrm{e}^{+}$and $V_{1}, \bar{f}_{4},[0: 360]$ degrees, \\
\hline dat. 23 & angle between decay planes of $V_{2}, f_{2}=\mathrm{e}^{-}$and $V_{2}, f_{5},[0: 360]$ degrees, \\
\hline dat. 24 & cosine of the angle between $f_{3}$ and $\bar{f}_{4},[-1: 1]$, \\
\hline dat. 25 & cosine of the angle between $f_{5}$ and $\bar{f}_{6},[-1: 1]$, \\
\hline dat. 26 & cosine of the angle between $V_{1}$ and $\bar{f}_{1}=\mathrm{e}^{+}$in the CM frame of $V_{1}$ and $V_{2},[-1: 1]$, \\
\hline dat. 27 & cosine of the angle between $V_{2}$ and $f_{2}=\mathrm{e}^{-}$in the CM frame of $V_{1}$ and $V_{2},[-1: 1]$. \\
\hline
\end{tabular}

Table 8

Description of the data files for the differential cross sections to $\bar{f}_{1} f_{2} \rightarrow V_{1} V_{2} \rightarrow f_{3} \bar{f}_{4} f_{5} \bar{f}_{6}(\gamma)$ where $\bar{f}_{1}=\mathrm{e}^{+}, f_{2}=\mathrm{e}^{-}, V_{1} \rightarrow f_{3} \bar{f}_{4}$, and $V_{2} \rightarrow f_{5} \bar{f}_{6}$ and the $\mathrm{e}^{+}$beam goes in the positive $z$ direction. All histograms are defined with 50 bins. 
Ref. [6], respectively.

In the subroutine SETTINGS in public.f the user can define additional histograms as described at the end of SETTINGS. The histograms are filled in

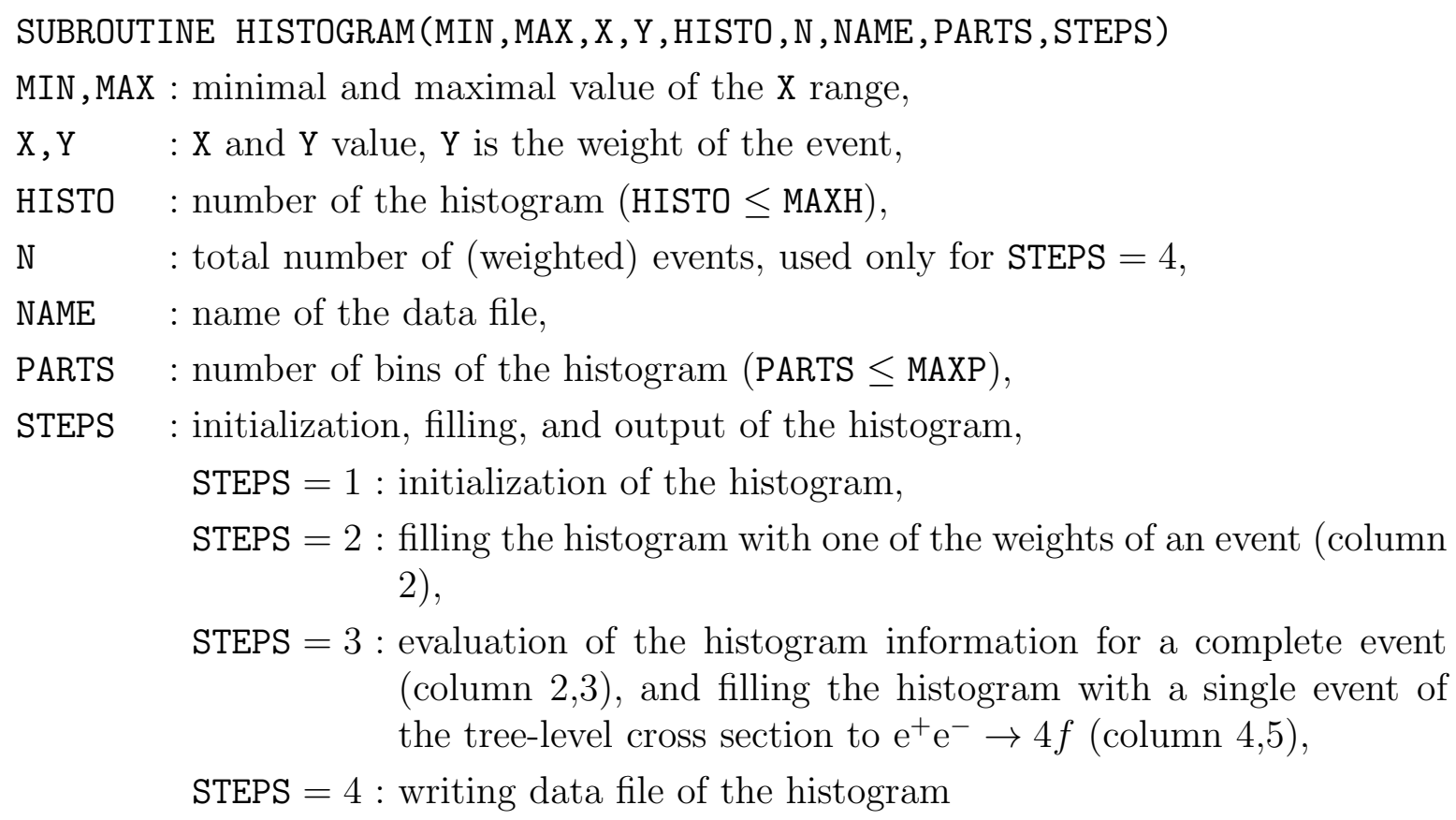

at the end of public.f. The parameters MAXH and MAXP specify the maximal number of histograms and bins and are set to $\mathrm{MAXH}=50$ and MAXP $=1000$, respectively. If more histograms or bins are required, these parameters have to be increased.

For the subtraction branch the splitting into STEPS $=2$ and STEPS $=3$ is needed since the weights for the bremsstrahlung process $\mathrm{e}^{+} \mathrm{e}^{-} \rightarrow 4 f \gamma$ and the corresponding subtraction functions are related to different phase-space points and, hence, cannot be combined into a single weight. In the soft and collinear limits the momenta corresponding to these different weights approach each other and the weights approximatively cancel, leading to IR-safe observables. These cancellations of the soft and collinear singularities occur within single histogram bins. Therefore, the histograms must be filled in a first step (STEPS $=2$ ) with all subcontributions corresponding to one weighted event. From the resulting contributions to each histogram bin the corresponding statistical errors can be calculated (STEPS $=3$ ).

In addition, for SPAW $=1$ the HBOOK file pawplot.paw is generated. It contains the histograms for the distributions as specified in pawgraphs.f. In racoonww.f the subroutine SETUP_GRAPHS is called where the histograms are defined (HBOOK). The subroutine GRAPHS is called in racoonww.f after the weights have been calculated. There the observables are defined and the corresponding histograms are filled (HFILL). In addition three more subroutines, GRAPHS_B, GRAPHS_K, and GRAPHS_R, are provided which generate histograms showing the distributions separately for the tree-level $\mathrm{e}^{+} \mathrm{e}^{-} \rightarrow 4 f$ contribution, the whole $4 f$ part, and the $4 f \gamma$ part. As usual, the histograms can be viewed by opening the PAW [45] main browser, and loading the file pawplot.paw. 


\subsection{The unweighting procedure and the interface with PYTHIA}

As default, RACOONWW generates only weighted events. For $\mathrm{SRC}=1$ and $\mathrm{SMC}=3$, or for $\mathrm{SRC}=0,2,3, \mathrm{RACOONWW}$ can generate unweighted events. The unweighting procedure is activated as follows:

- The number of generated unweighted events NEVENTSUNW must be set in the main program in racoonww.f. By default NEVENTSUNW $=0$, and no unweighted events are generated. In the RC mode, to obtain a precision comparable to the "best" result of Ref. [6], we recommend to use NEVENTSUNW $=5 \times 10^{4}$. With an efficiency of one unweighted event per $10^{3}$ weighted events, this corresponds to the calculation of approximately $5 \times 10^{7}$ weighted events. In the tree-level and IBA modes the efficiency is about a factor 100 better and we recommend to use NEVENTSUNW $=5 \times 10^{5}$. Note that for certain processes the efficiency can strongly depend on the separation cuts.

- The number of weighted events (NEVENTSW) that are used only to determine the maximal weights for the hit-and-miss algorithm is set in the input file. This should be much smaller than what is usually used without unweighting, for instance, we recommend NEVENTSW $=10^{5}$.

When including radiative corrections $(\mathrm{SRC}=1)$, only the branch $\mathrm{SMC}=3$ can be used in the unweighting procedure.

The unweighted events are generated in the subroutine EVENTGENERATION in racoonww.f from weighted events with a hit-and-miss algorithm. More precisely, for each weighted event an unweighted event is generated if the corresponding weight is larger than the product of a random number RAND $(0 \leq$ RAND $\leq 1)$ and the maximal weight. Otherwise no unweighted event is generated. The maximal weight, WEIGHTTOTMAX, is calculated by multiplying the maximal weight of the first NEVENTSW events with a factor two. The number NEVENTSW and the factor can be tuned to suppress weights that are larger than WEIGHTTOTMAX in the main calculation. If a weight exceeds the maximal weight, WEIGHTTOTMAX is redefined by the value of the weight and a warning is written in the output file.

The maximal weight of the bremsstrahlung contribution exceeds by far the maximal weights of the other subcontributions. Therefore the other weights are not calculated for each event in order to enhance the efficiency of the unweighting procedure. They are calculated only all NWEIGHT times but are multiplied by NWEIGHT. In this way the probability of an unweighted event from the different subcontributions remains unchanged. The integers NWEIGHT $(1: 29)$ are chosen in such a way that each subcontribution leads to roughly the same maximal weight, which has to be smaller than WEIGHTTOTMAX.

The information about the (unweighted) event is provided in the HEP standard format in the common block HEPEVT [24]. The parameters and flags of the common block HEPEVT are set in the subroutine EVENTGENERATION in racoonww.f. For a detailed description we refer to Ref. [23], p. 63. While the first six particle entries in HEPEVT are the external 
fermions, $\mathrm{e}^{+} \mathrm{e}^{-} \rightarrow f_{3} \bar{f}_{4} f_{5} \bar{f}_{6}$, the last up to two entries are filled by a possible hard photon from the bremsstrahlung process $\mathrm{e}^{+} \mathrm{e}^{-} \rightarrow 4 f \gamma$ and, if higher-order LL corrections from ISR are included, a photon collinear to the beam. The four-momentum of the ISR photon collinear to the beam is calculated from the four-momenta of the other external particles using momentum conservation. Note that the positron beam goes in $+z$ direction and that cuts and photon recombination influence the resulting unweighted events.

Although negative weights appear in the $\mathrm{RC}$ mode also for SRC $=3$, they have only a negligible influence on the observables that we have studied so far. Nevertheless, RACOONWW also unweights the events with negative weights, but does not fill the HEPEVENT common block with these weights. The resulting unweighted negative-weight events can, for instance, be used to estimate the uncertainty resulting from discarding negative weights.

In the output file, RACOONWW provides the number of unweighted events, the number of unweighted events with negative weights, and the number of events that enforce a redefinition of the maximal weight. Note that the result for the total cross section and for the distributions are always calculated from the weighted events.

To include parton shower and hadronization for coloured final states, RACOONWW provides an interface with PYTHIA for unweighted events by calling the PYTHIA subroutine PY4FRM (see p. 236 of Ref. [23]):

SUBROUTINE PY4FRM (ATOTSQR , A1SQR , A2SQR, ISTRAT , IRAD , ITAU , ICOM)

ATOTSQR : total matrix-element squared for the event calculated in the subroutine EVENTGENERATION as ATOTSQR = ATOTSQ(NS), where ATOTSQ(NS) is the matrix element squared of the NS-th weight WEIGHT(NS),

A1SQR : matrix-element squared for the configuration when $f_{3}, \bar{f}_{4}$ and $f_{5}, \bar{f}_{6}$ are colour singlets $(\mathrm{A} 1 \mathrm{SQR}=\mathrm{A} 1 \mathrm{SQ}(\mathrm{NS}))$,

A2SQR : matrix-element squared for the configuration when $f_{3}, \bar{f}_{6}$ and $f_{5}, \bar{f}_{4}$ are colour singlets $(\mathrm{A} 2 \mathrm{SQR}=\mathrm{A} 2 \mathrm{SQ}(\mathrm{NS}))$,

ISTRAT : flag for the strategy to select either of both colour configurations; we choose ISTRAT $=0$,

IRAD : flag for final-state photon radiation in PYTHIA. As default, photon radiation is switched off $(\mathrm{IRAD}=0)$ to avoid double counting in the $\mathrm{RC}$ mode $(\mathrm{SRC}=$ 1 ); in the tree-level and IBA modes IRAD $=1$ may be chosen,

ITAU : flag for the handling of $\tau$-lepton decays; by default $\tau$ decays are not included $($ ITAU $=0)$, since the final-state fermions are considered to be massless in RACOONWW; however, RACOONWW provides a subroutine to generate a massive two-particle phase space (see below), so that $\tau$ decays can be taken into account $($ ITAU $=1)$,

ICOM : flag for the place where the information about the event is stored; by default $\mathrm{ICOM}=0$ in order to store the information in the HEPEVT common block.

The amplitudes ATOTSQ (1:8), A1SQ(1:8), A1SQ(1:8), corresponding to the weights in Tables 5, 6, and 7, are calculated by using ATOTSQ4, A1SQ4, A2SQ4 for the tree-level pro- 
cesses $\mathrm{e}^{+} \mathrm{e}^{-} \rightarrow 4 f$ and by ATOTSQ5, A1SQ5, A2SQ5 for the tree-level processes $\mathrm{e}^{+} \mathrm{e}^{-} \rightarrow 4 f \gamma$. The colour amplitudes are calculated in the functions M2_EPEM_4F and M2_EPEM_4FA in ee4fa_amps.f and stored in the common blocks COLAMP and COLAMPA.

As default, this interface is not active in RACOONWW. It can be activated as follows:

- Link Pythia by adding the file with the Pythia code, e.g. pythia1568.f of version 6.1, to the list of objects OBJS in the makefile. PYTHIA can be downloaded from http://www.thep.lu.se/ torbjorn/Pythia.html.

- Uncomment the line with the call of PY4FRM in the main program in racoonww.f.

Note that the compilation of Pythia needs extensive memory.

As mentioned above, RACOONWW provides the possibility of converting pairs of massless momenta to massive momenta by calling

SUBROUTINE MASSIVE(M1, M2, PHEP1, PHEP2)

M1, M2 : masses of the two final-state particles,

PHEP1, PHEP2 : four momenta of the two final-state particles with zero masses before the call of MASSIVE and with masses M1,M2 after the call of MASSIVE

in racoonww.f. This could be used to switch to massive kinematics for the $\tau$ lepton before interfacing with PyтHIA. Note that the momenta of two final-state particles have to be changed in order to maintain four-momentum conservation and the mass-shell conditions.

\section{Summary}

We have described the MC generator RACOONWW, version 1.3, for four-fermion production, off-shell $\mathrm{W}$-pair production, as well as the radiative processes $\mathrm{e}^{+} \mathrm{e}^{-} \rightarrow 4 f \gamma$. At tree level, the complete set of diagrams is included. For $\mathrm{e}^{+} \mathrm{e}^{-} \rightarrow \mathrm{WW} \rightarrow 4 f$, the prediction is based on the complete $\mathcal{O}(\alpha)$ corrections in double-pole approximation and QED initialstate radiation up to $\varnothing\left(\alpha^{3}\right)$. The corresponding theoretical uncertainty is at the level of $0.5 \%$ for the total cross section and of the order of $1 \%$ for distributions in the energy range between $\sim 170$ and $500 \mathrm{GeV}$. The use of a double-pole approximation, in general, puts a restriction on the theoretical uncertainty at the level of a few per mil away from threshold. RACOONWW also provides an improved-Born approximation with an uncertainty of $2 \%$, which is also applicable in the threshold region. More precise calculations require the inclusion of radiative corrections beyond double-pole approximation. 


\section{Acknowledgements}

We are grateful to D. Graudenz and R. Pittau for useful discussions, and to T. Sjöstrand and S. Roth for their help in implementing the interface to PYTHIA. We thank the authors of Ref. [11], in particular A. Chapovsky, and the authors of YFSWW3, in particular, W. Płaczek, for useful numerical comparisons. We are indebted to R. Chierici, F. Cossutti, L. Malgeri, K. Rabbertz, A. Strässner, and M. Thomson for valuable feedback concerning the performance of the code and to all members of the LEP2 WW/4f working group, in particular E. Lançon, R. Tanaka, A. Valassi, and M. Verzocchi for many useful discussions.

This work has been partly supported by the European Commission 5th framework contract HPRN-CT-2000-00149, the Swiss Bundesamt für Bildung und Wissenschaft contract 99.0043, and the US DOE Contract DE-FG02-91ER40685. S.D. is a Heisenberg Fellow of the Deutsche Forschungsgemeinschaft

\section{A Sample runs and output}

Two test runs are discussed in the following. One is a run of RACOONWW in the RC mode. The second one is the calculation of the cross section to $\mathrm{e}^{+} \mathrm{e}^{-} \rightarrow 4 f \gamma$ within the IBA mode. Both test runs and three additional sample runs are discussed in the file README.

\section{A.1 Test run in the $R C$ mode}

The first test run calculates the "best" predictions for the cross section to the process $\mathrm{e}^{+} \mathrm{e}^{-} \rightarrow \mathrm{u} \overline{\mathrm{d}} \mu^{-} \bar{\nu}_{\mu}(\gamma)$ for a CM energy of $\sqrt{s}=200 \mathrm{GeV}$. Thereby, the full $\varnothing(\alpha)$ corrections in DPA and leading higher-order corrections are included, and the W-boson width is calculated. The recombination scheme and separation cuts of Ref. [6] are applied. The numerical integration is performed in the slicing branch with $5 \times 10^{7}$ (weighted) events. On a DEC/ALPHA workstation the computing time is about 30 hours.

\section{A.1.1 Input file inputsli}

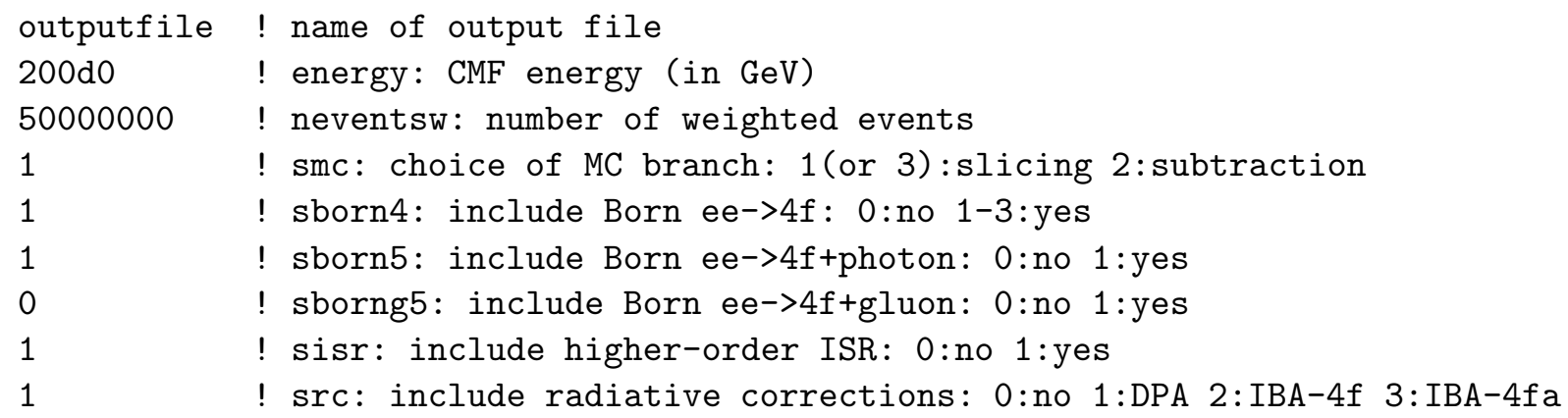




\begin{tabular}{|c|c|}
\hline 0 & ! scoultree: Coulomb singularity for ee->4f,4f+ga: $0:$ no 1,2 :yes \\
\hline 3 & ! qnf: Coulomb singularity for ee->4f (DPA): 1,2 , or 3 \\
\hline 1 & ! qreal: neglect imaginary part of virt. corr.: $0:$ no $1:$ yes \\
\hline 2 & ! qalp: choice of input-parameter scheme: 0,1 , or 2 \\
\hline 4 & ! qgw: calculate the W-boson width: $0:$ no 1-4:yes \\
\hline 1 & ! qprop: choice of width scheme: $0,1,2,3$ or 4 \\
\hline 0 & ! ssigepem4: choice of diag. for Born ee->4f: 0:all 1-5:subsets \\
\hline 5 & ! ssigepem5: choice of diag. for Born ee->4f+ga: 0:all 1-5:subsets \\
\hline 0 & ! ssigepemg5: choice of diag. for Born ee->4f+gl: $0:$ all 1,5:subsets \\
\hline 2 & ! qqcd: include QCD radiative corr.: 0: no 1:CC03 2: naive 3:CC11 \\
\hline 0 & ! sqcdepem: include gluon-exch. diag. in Born: 0:no 1:yes 2:only \\
\hline $\mathrm{u}$ & ! fermion 3 \\
\hline $\mathrm{d}$ & ! anti-fermion 4 \\
\hline $\mathrm{mu}$ & ! fermion 5 \\
\hline nu_mu & ! anti-fermion 6 \\
\hline OdO & ! pp: degree of positron beam polarization $[\$-1 \$ \mathrm{~d} 0: 1 \mathrm{~d} 0]$ \\
\hline OdO & ! pm: degree of electron beam polarization $[\$-1 \$ \mathrm{~d} 0: 1 \mathrm{~d} 0]$ \\
\hline 1 & ! srecomb: recombination cuts: $0:$ no $1:$ TH $2:$ EXP \\
\hline $5 \mathrm{~d} 0$ & ! precomb(1): angular rec. cut between photon and beam \\
\hline $1 \mathrm{~d} 0$ & ! precomb(2): rec. cut on photon energy \\
\hline $5 \mathrm{~d} 0$ & ! precomb(3): inv.-mass rec. (TH) or angular rec. cut for lept. (EXP) \\
\hline OdO & ! precomb(4): angular rec. cut for quarks(EXP) \\
\hline 0 & ! srecombg: gluon recombination cuts: $0:$ no $1:$ TH $2:$ EXP \\
\hline OdO & ! precombg(1): rec. cut on gluon energy \\
\hline OdO & ! precombg(2): inv.-mass (TH) or angular (EXP) recombination cut \\
\hline 0 & ! satgc: anomalous triple gauge couplings (TGC): $0:$ no $1:$ yes \\
\hline OdO & ! TGC Delta g_1^A \\
\hline OdO & ! TGC Delta g_1^Z \\
\hline OdO & ! TGC Delta kappa`A \\
\hline OdO & ! TGC Delta kappa^Z \\
\hline OdO & ! TGC lambda^A \\
\hline OdO & ! TGC lambda^Z \\
\hline Od0 & ! TGC g_4^A \\
\hline OdO & ! TGC $g_{-} 4^{\wedge} Z$ \\
\hline Odo & ! TGC g_5^A \\
\hline odo & ! TGC g_5^Z \\
\hline OdO & ! TGC tilde kappa`A \\
\hline Od0 & ! TGC tilde kappa`Z \\
\hline Odo & ! TGC tilde lambda^A \\
\hline OdO & ! TGC tilde lambda`Z \\
\hline Od0 & ! TGC f_4^A \\
\hline odo & ! TGC f_4^Z \\
\hline Odo & ! TGC f_5^A \\
\hline odo & ! TGC f_5^Z \\
\hline Odo & ! TGC h_1^A \\
\hline OdO & ! TGC h_1^Z \\
\hline OdO & ! TGC h_3^A \\
\hline OdO & ! TGC h_3^Z \\
\hline
\end{tabular}




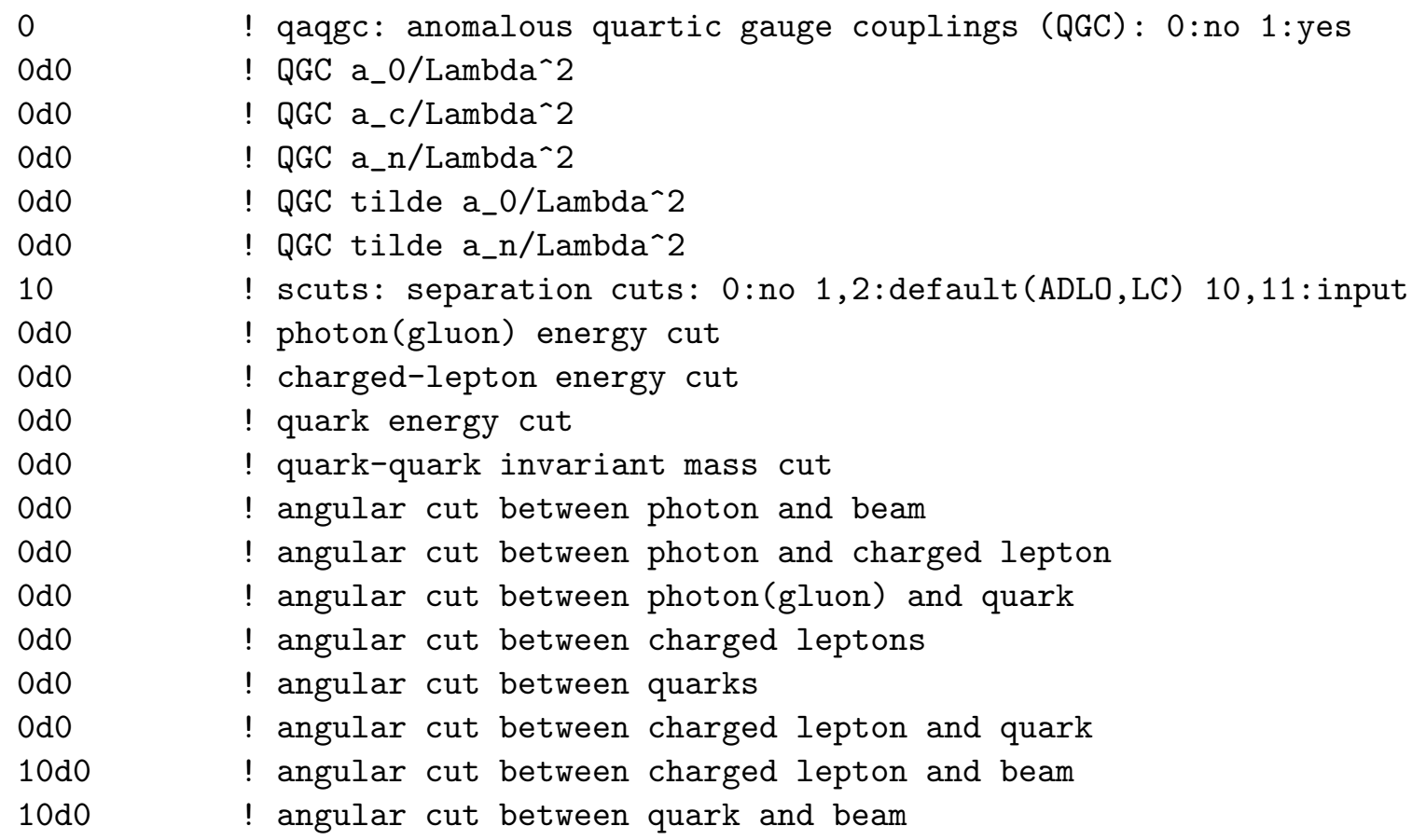

\section{A.1.2 Output file}

smc $=1:$ Phase-space-slicing branch of RacoonWW

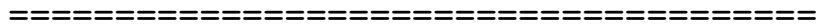

$$
\begin{array}{rr}
\text { technical cutoff parameters (photon): } \\
\text { delta_s }= & 1.000000000000000 \mathrm{E}-003 \\
\text { delta_c } & 5.000000000000000 \mathrm{E}-004
\end{array}
$$

Input parameters:

$$
\begin{aligned}
& \text { CMF energy }=200.00000 \mathrm{GeV}, \quad \text { Number of events }=50000000 \text {, } \\
& \operatorname{alpha}(0)=1 / 137.0359895, \text { alpha }(M Z)=1 / 128.88700, \text { alpha_s }=0.11900, \\
& \mathrm{GF}=.1166370 \mathrm{E}-04 \text {, } \\
& \text { MW }=80.35000, \quad M Z=91.18670, \quad M H=150.00000, \\
& \mathrm{GW}=2.08699, \quad \mathrm{GZ}=2.49471, \\
& \mathrm{me}=.51099907 \mathrm{E}-03, \quad \mathrm{mmu}=0.105658389, \operatorname{mtau}=1.77705, \\
& \mathrm{mu}=0.00485, \quad \mathrm{mc}=1.55000, \quad \mathrm{mt}=174.17000, \\
& \mathrm{md}=0.00485, \quad \mathrm{~ms}=0.15000, \quad \mathrm{mb}=4.50000 \text {. }
\end{aligned}
$$


$\mathrm{pp}=0.0$ : degree of positron beam polarization.

$\mathrm{pm}=0.0$ : degree of electron beam polarization.

qalp $=2:$ GF-parametrization scheme.

qgw = 4: one-loop W-boson width calculated (with QCD corr.).

qprop= 1: constant width.

sborn4= 1: tree-level process ee $\rightarrow 4 f$.

ssigepem4= 0: all electroweak diagrams included.

qqcd= 2: naive QCD corrections included.

src= 1: virtual corrections in DPA and real corrections included. ssigepem5 $=5$ : real photon corr. : only CC11 class of diagrams included.

qqcd= 2: naive QCD corrections included

qreal= 1: imaginary part of virtual corrections neglected.

qnf $=3$ : off-shell Coulomb singularity with off-shell Born included.

sisr= 1: initial-state radiation up to order alpha^3 included.

srecomb= 1: with photon recombination:

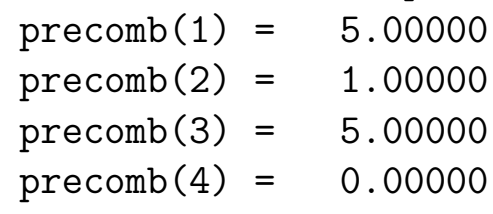

scuts=10: with separation cuts:

angle $(1,3)>10.00000 \mathrm{deg}$

angle $(1,4)>10.00000 \mathrm{deg}$

angle $(1,5)>10.00000 \mathrm{deg}$

angle $(2,3)>10.00000 \mathrm{deg}$

angle $(2,4)>10.00000 \mathrm{deg}$

angle $(2,5)>10.00000 \mathrm{deg}$

\begin{tabular}{|c|c|c|c|c|c|c|c|}
\hline & & & & & & ry & \\
\hline 00 & & 92.61877 & +- & 7251 & & $2.61877+-$ & 2.47251 \\
\hline 00 & & 383 & +- & 1.89683 & & $130+-$ & 815 \\
\hline 3000000 & & 445 & +- & 1.89273 & & $02+-$ & 535 \\
\hline 4000000 & & 6888 & +- & 1.80112 & & $6648+-$ & 1.01667 \\
\hline 5000 & & 590 & +- & 1.65706 & & $2837+-$ & 826 \\
\hline 600 & & 34 & +- & 2.2 & & $70+-$ & 13 \\
\hline 700 & & 94.42877 & +- & 1.76546 & & $92.16842+-$ & 684 \\
\hline 8000 & & 65 & +- & 1.68242 & & $91.89757+-$ & 3649 \\
\hline 9000 & & 677 & +- & 1.59058 & & $63637+-$ & 0.6 \\
\hline 000 & & 9013 & +- & 1.90289 & & $.97175+-$ & 260 \\
\hline 100 & & 66 & +- & 1.8 & & $474+-$ & 0.57233 \\
\hline 2000 & & 593.04183 & - & 1.90 & & $591.77033+-$ & 0.54801 \\
\hline 20 & & 15 & . & 1.6 & & $.81624+-$ & 0.5 \\
\hline
\end{tabular}




\begin{tabular}{|c|c|c|c|c|c|c|c|c|}
\hline & & & +- & & & & +- & \\
\hline & & 1.71289 & +- & 79049 & & 1.70689 & +- & \\
\hline 000 & & 1.53899 & +- & 6907 & & 91.69640 & +- & 402 \\
\hline 000 & & 7.50308 & +- & 58333 & & 44973 & +- & 54 \\
\hline & & 9929 & +- & 50 & & 49 & +- & 50 \\
\hline 000 & & & +- & .73210 & & & +- & \\
\hline & & 324 & +- & .74407 & & & +- & 647 \\
\hline 000 & & 4008 & +- & .80517 & & 99 & +- & 9655 \\
\hline 0000 & & 2.44203 & +- & .72115 & & 31 & +- & 38652 \\
\hline & & 38 & +- & 91 & & & +- & \\
\hline & & & +- & & & & +- & 902 \\
\hline 00 & & 5275 & +- & 673 & & 47 & +- & 48 \\
\hline & & 325 & +- & & & & +- & 32 \\
\hline 0 & & 1.22938 & +- & 30 & & 13 & +- & 651 \\
\hline & & 261 & +- & & & & +- & \\
\hline & & & +- & & & & +- & \\
\hline 00 & & 9423 & +- & 95 & & 06 & +- & 740 \\
\hline & & 12 & +- & & & & +- & 60 \\
\hline & & 75 & +- & & & & +- & 589 \\
\hline & & 952 & +- & & & & +- & 012 \\
\hline & & & +- & & & & +- & \\
\hline & & 6319 & +- & & & & +- & 089 \\
\hline & & 598 & +- & & & 42 & +- & 9623 \\
\hline & • & & +- & & & & +- & \\
\hline & & 74 & +- & & & 37 & +- & 8849 \\
\hline & & 522 & +- & 1. & & 57 & +- & 427 \\
\hline & & 906 & +- & & & & +- & .28016 \\
\hline & & 3361 & +- & 1. & & 5 & +- & 0.27732 \\
\hline & & & +- & & & & +- & 0.27371 \\
\hline & & 329 & +- & & & 74 & +- & .27003 \\
\hline 00000 & & 48 & +- & 1.6 & & 43 & +- & 0.26641 \\
\hline & & .68588 & +- & & & & +- & 0.26294 \\
\hline & & .26056 & +- & $1 . \varepsilon$ & & & +- & 0.26021 \\
\hline & & & +- & & & & +- & 0.25711 \\
\hline & & .22822 & +- & 1.6 & & 428 & +- & 0.25409 \\
\hline & & 591.98897 & +- & 1.63064 & & 591.23009 & +- & 0.25112 \\
\hline חתחמחת & & 93.47276 & +- & 1.71196 & & 591.27494 & +- & 0.2484 \\
\hline
\end{tabular}

Result:

$\begin{array}{lrl}\text { Number of weighted events } & =50000000 \\ \text { Average } & 591.2749427045 \mathrm{fb} \\ \text { Standard deviation } & = & 0.2484667990 \mathrm{fb} \\ \text { Maximal weight } & = & 0.0287650976 \mathrm{fb}\end{array}$


Subcontributions without naive QCD:

$\begin{array}{llr}\text { Tree-level four-fermion cross section: } & = & 627.6779997923 \mathrm{fb} \\ \text { Average } & & 0.0003787331 \mathrm{fb} \\ \text { Standard deviation } & & 0.1075731739 \mathrm{fb} \\ \text { Maximal weight } & & \\ & & \\ \text { 2->4 DPA part : } & & \\ \text { Average } & & \\ \text { Standard deviation } & = & 0.0030507290 \mathrm{fb} \\ \text { Maximal weight } & = & 0.0000240297 \mathrm{fb}\end{array}$

2->4 virtual+soft+final-state collinear photon part:

without LL O(alpha) ISR

$\begin{array}{lrr}\text { Average } & & -67.4968488454 \mathrm{fb} \\ \text { Standard deviation } & = & 0.0117625527 \mathrm{fb} \\ \text { Maximal weight } & =0.0000250099 \mathrm{fb}\end{array}$

2->4 initial-state collinear photon part $\left(\mathrm{e}^{+}\right)$:

without LL O(alpha) ISR

$\begin{array}{llr}\text { Average } & & -65.5094255283 \mathrm{fb} \\ \text { Standard deviation } & = & 0.0131360874 \mathrm{fb} \\ \text { Maximal weight } & =0.0000551979 \mathrm{fb}\end{array}$

2->4 initial-state collinear photon part $\left(\mathrm{e}^{-}\right)$:

without LL O(alpha) ISR

$\begin{array}{llr}\text { Average } & & -65.4479754318 \mathrm{fb} \\ \text { Standard deviation } & =0.0131306505 \mathrm{fb} \\ \text { Maximal weight } & =0.0000523650 \mathrm{fb}\end{array}$

2->4 LL initial-state radiation up to 0(alpha^3):

Average $\quad=\quad-54.3690820517 \mathrm{fb}$

Standard deviation $=0.0645784370 \mathrm{fb}$

Maximal weight $=0.0003228727 \mathrm{fb}$

2->5 Bremsstrahlung contribution (QED):

Average $\quad=201.0338802635 \mathrm{fb}$

Standard deviation $=0.2234350653 \mathrm{fb}$

Maximal weight $=0.0277012770 \mathrm{fb}$

2->5 Bremsstrahlung contribution (QCD):

Average $=0.0000000000 \mathrm{fb}$

Standard deviation $=0.0000000000 \mathrm{fb}$

Maximal weight $=0.0000000000 \mathrm{fb}$ 
$\%$ events rejected in calculation of weight( 1$): 5.3 \%$

$\%$ events rejected in calculation of weight( 2$): 5.2 \%$

$\%$ events rejected in calculation of weight( 3$): 5.1 \%$

$\%$ events rejected in calculation of weight( 4$): 6.1 \%$

$\%$ events rejected in calculation of weight ( 5): $6.2 \%$

$\%$ events rejected in calculation of weight ( 6$): 6.0 \%$

$\%$ events rejected in calculation of weight $(7): 34.6 \%$

$\%$ events rejected in calculation of weight( 8$): 0.0 \%$

\section{A.2 Test run in the IBA mode for the processes $\mathrm{e}^{+} \mathrm{e}^{-} \rightarrow 4 f \gamma$}

The second test run calculates the IBA for the process $\mathrm{e}^{+} \mathrm{e}^{-} \rightarrow \mathrm{u} \overline{\mathrm{d}} \mu^{-} \bar{\nu}_{\mu} \gamma$ in the subtraction branch for a CM energy of $\sqrt{s}=200 \mathrm{GeV}$. The ADLO cuts are applied, and the IBA Wboson width is calculated. On a DEC/ALPHA workstation the computing time is about 2 hours.

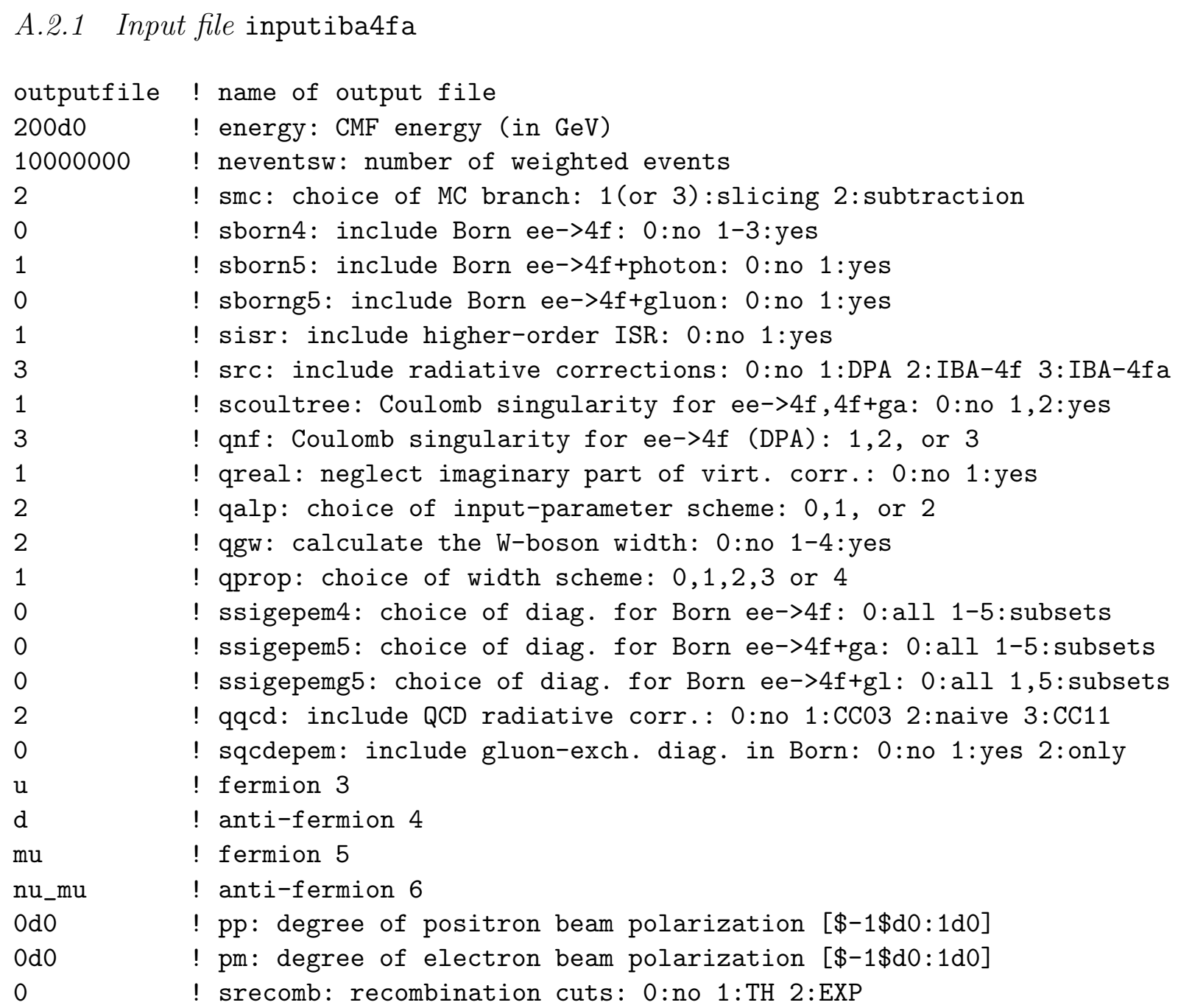




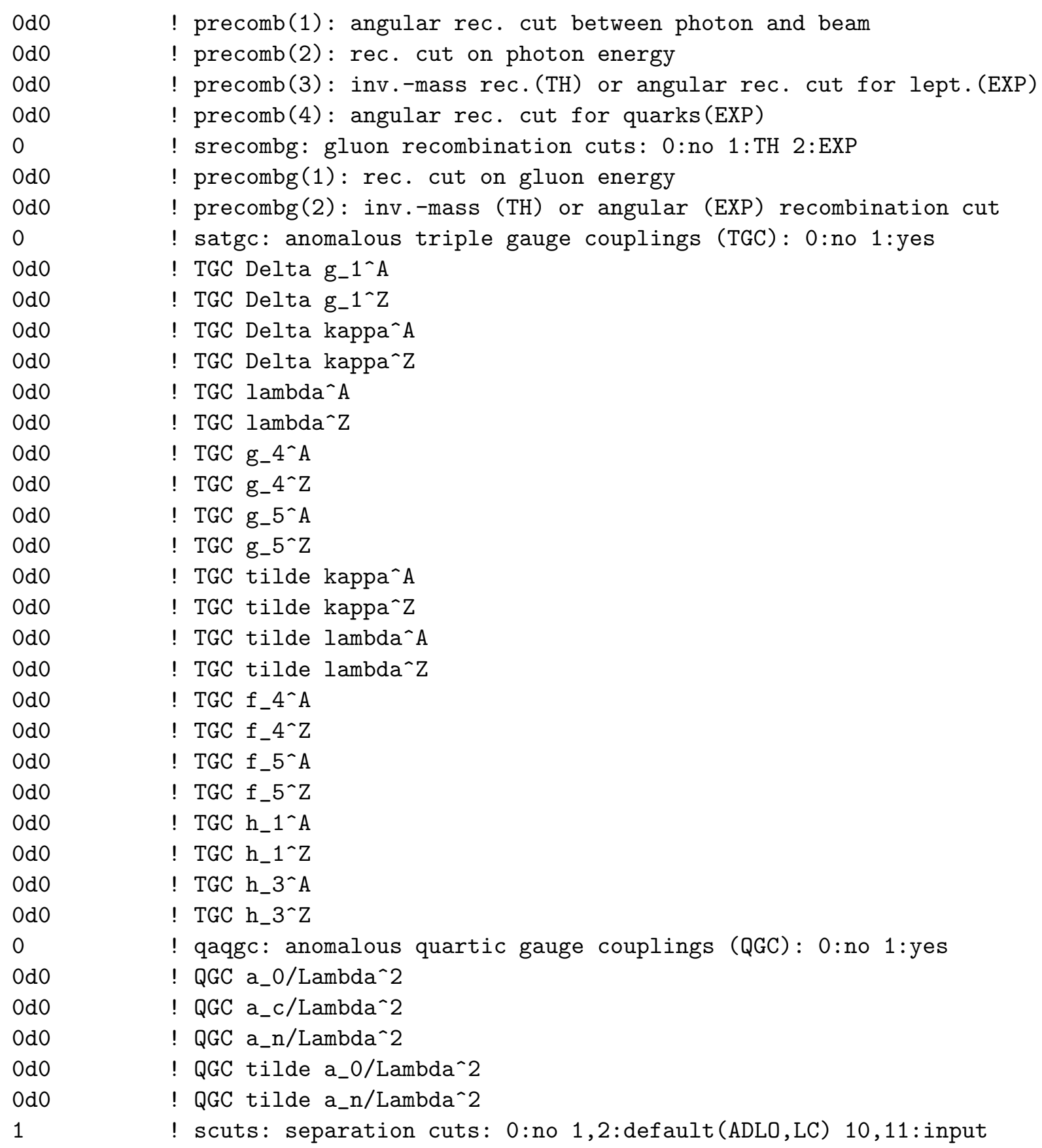

\section{A.2.2 Output file}

smc $=2$ : Subtraction-method branch of RacoonWW

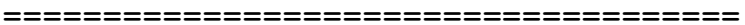

Input parameters:

$\mathrm{CMF}$ energy $=200.00000 \mathrm{GeV}, \quad$ Number of events $=10000000$, 


$$
\begin{aligned}
& \text { alpha }(0)=1 / 137.0359895, \text { alpha }(M Z)=1 / 128.88700, \text { alpha_s }=0.11900, \\
& \mathrm{GF}=.1166370 \mathrm{E}-04 \text {, } \\
& \mathrm{MW}=80.35000, \quad \mathrm{MZ}=91.18670, \quad \mathrm{MH}=150.00000, \\
& \mathrm{GW}=2.09436, \quad \mathrm{GZ}=2.49471 \text {, } \\
& \mathrm{me}=.51099907 \mathrm{E}-03, \quad \mathrm{mmu}=0.105658389, \mathrm{mtau}=1.77705, \\
& \mathrm{mu}=0.00485, \quad \mathrm{mc}=1.55000 \quad \mathrm{mt}=174.17000, \\
& \mathrm{md}=0.00485, \quad \mathrm{~ms}=0.15000, \quad \mathrm{mb}=4.50000 .
\end{aligned}
$$

Effective branching ratios:

leptonic $\mathrm{BR}=0.32512$, hadronic $\mathrm{BR}=0.67488$, total $\mathrm{BR}=1.00000$

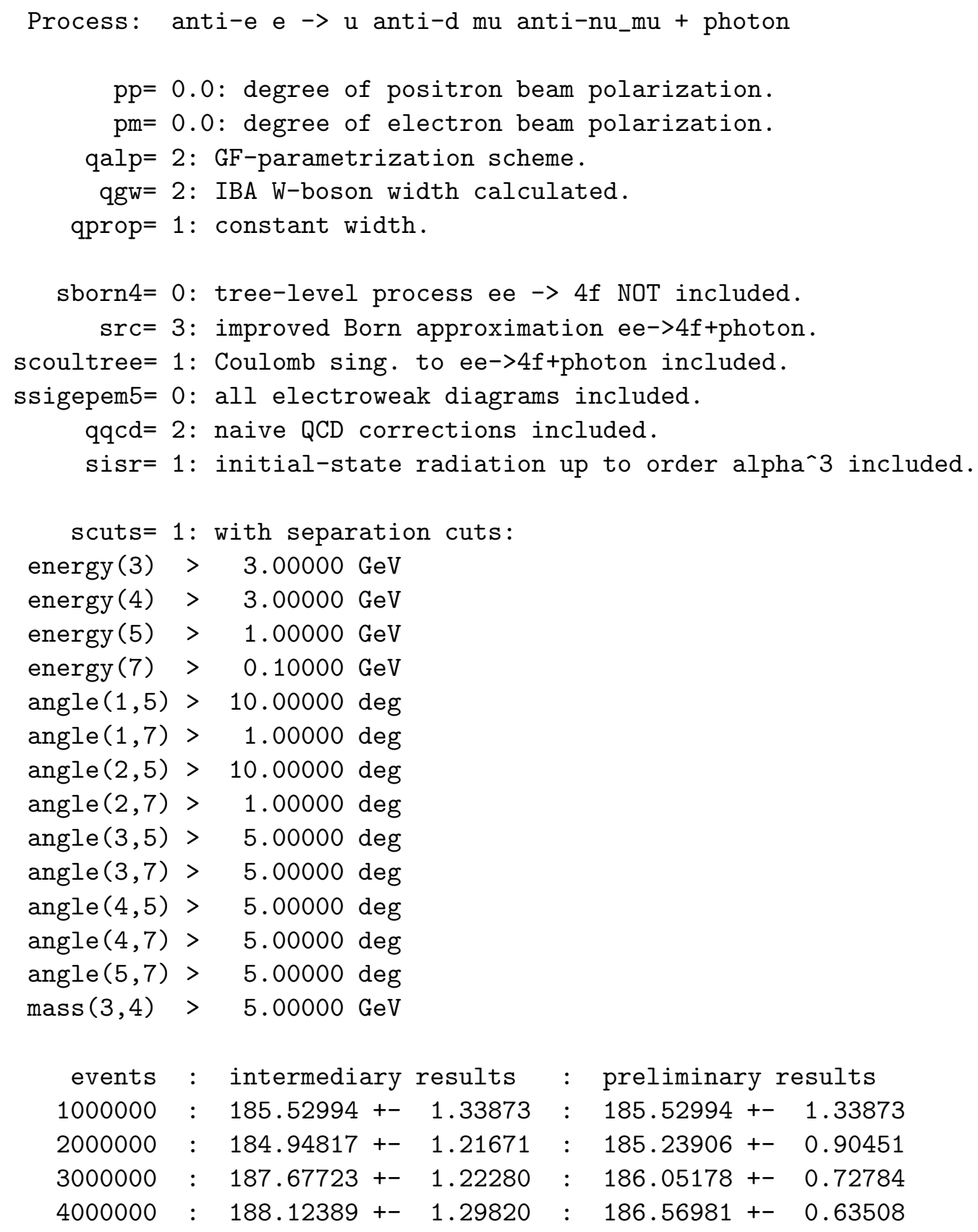




$\begin{array}{rllllll}5000000 & : & 186.24278+- & 1.21187 & : & 186.50440+- & 0.56291 \\ 6000000 & : & 184.67305+- & 1.27906 & : & 186.19918+- & 0.51526 \\ 7000000 & : & 186.27700+- & 1.29118 & : & 186.21029+- & 0.47862 \\ 8000000 & : & 188.42698+- & 1.29575 & : & 186.48738+- & 0.44903 \\ 9000000 & : & 188.10039+- & 1.28908 & : & 186.66660+- & 0.42406 \\ 10000000 & : & 187.29950+- & 1.27811 & : & 186.72989+- & 0.40248\end{array}$

Result :

$\begin{array}{llll}\text { Number of weighted events } & = & 10000000 \\ \text { Average } & 186.7298922322 \mathrm{fb} \\ \text { Standard deviation } & = & 0.4024824609 \mathrm{fb} \\ \text { Maximal weight } & = & 0.0452822218 \mathrm{fb}\end{array}$

Subcontributions without naive QCD:

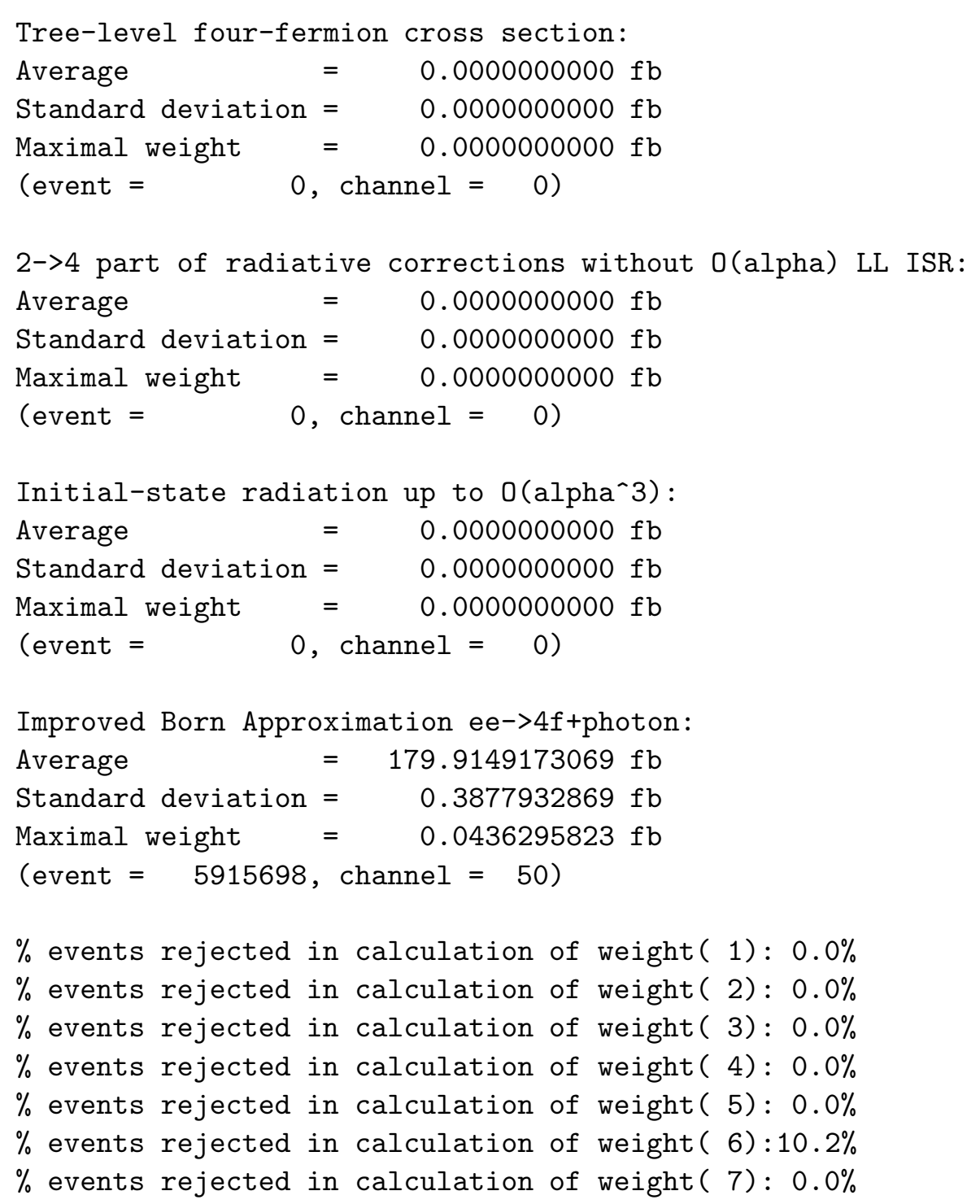


$\%$ events rejected in calculation of weight(29): $0.0 \%$

\section{References}

[1] The LEP Collaborations ALEPH, DELPHI, L3, OPAL, the LEP Electroweak Working Group, and the SLD Heavy Flavour and Electroweak Groups, CERN-EP-2001-098, hep$\mathrm{ex} / 0112021$.

[2] E. Accomando et al. [ECFA/DESY LC Physics Working Group Collaboration], Phys. Rept. 299 (1998) 1 hep-ph/9705442;

J. A. Aguilar-Saavedra et al., TESLA Technical Design Report Part III: Physics at an $\mathrm{e}^{+} \mathrm{e}^{-}$ Linear Collider, hep-ph/0106315;

[3] T. Abe et al. [American Linear Collider Working Group Collaboration], in Proc. of the APS/DPF/DPB Summer Study on the Future of Particle Physics (Snowmass 2001) ed. R. Davidson and C. Quigg, SLAC-R-570 Resource book for Snowmass 2001, hepex/0106055, hep-ex/0106056, hep-ex/0106057, hep-ex/0106058.

[4] A. Denner, S. Dittmaier, M. Roth and D. Wackeroth, Nucl. Phys. B 560 (1999) 33 hep$\mathrm{ph} / 9904472$.

[5] A. Denner, S. Dittmaier, M. Roth and D. Wackeroth, Phys. Lett. B 475 (2000) 127 hep$\mathrm{ph} / 9912261$ and hep-ph/9912447.

[6] A. Denner, S. Dittmaier, M. Roth and D. Wackeroth, Nucl. Phys. B 587 (2000) 67 hep$\mathrm{ph} / 0006307$.

[7] A. Denner, S. Dittmaier, M. Roth and D. Wackeroth, in Proc. of the 5th International Symposium on Radiative Corrections (RADCOR 2000) ed. H. E. Haber, hep-ph/0101257.

[8] A. Denner, S. Dittmaier, M. Roth and D. Wackeroth, Eur. Phys. J. C 20 (2001) 201 hep$\mathrm{ph} / 0104057$.

[9] W. Beenakker et al., in Physics at LEP2, eds. G. Altarelli, T. Sjöstrand and F. Zwirner (CERN 96-01, Geneva, 1996), Vol. 1, p. 79 hep-ph/9602351.

[10] M. W. Grünewald et al., in Reports of the Working Groups on Precision Calculations for LEP2 Physics, eds. S. Jadach, G. Passarino and R. Pittau (CERN 2000-009, Geneva, 2000), p. 1 [hep-ph/0005309].

[11] W. Beenakker, F. A. Berends and A. P. Chapovsky, Nucl. Phys. B 548, 3 (1999) hepph/9811481].

[12] S. Jadach, W. Płaczek, M. Skrzypek and B. F. Ward, Phys. Rev. D 54 (1996) 5434 hepph/9606429]; Phys. Lett. B 417 (1998) 326 hep-ph/9705429]; Phys. Rev. D 61 (2000) 113010 hep-ph/9907436]; Phys. Rev. D 65 (2002) 093010 hep-ph/0007012].

[13] G. Montagna, M. Moretti, O. Nicrosini, M. Osmo and F. Piccinini, Eur. Phys. J. C 21 (2001) 291 hep-ph/0103155. 
[14] C. G. Papadopoulos, Comput. Phys. Commun. 137 (2001) 247 hep-ph/0007335;

A. Kanaki and C. G. Papadopoulos, hep-ph/0012004.

[15] A. Denner, S. Dittmaier, M. Roth and D. Wackeroth, in Proc. of the International Europhysics Conference on High-Energy Physics (HEP 2001), hep-ph/0110402.

[16] D. Y. Bardin, A. Olshevsky, M. S. Bilenky and T. Riemann, Phys. Lett. B 308 (1993) 403 [Erratum-ibid. B 357 (1995) 725].

[17] A. Denner, S. Dittmaier and M. Roth, Nucl. Phys. B 519 (1998) 39 hep-ph/9710521.

[18] E. A. Kuraev and V. S. Fadin, Yad. Fiz. 41 (1985) 753 [Sov. J. Nucl. Phys. 41 (1985) 466]; G. Altarelli and G. Martinelli, in "Physics at LEP", eds. J. Ellis and R. Peccei, CERN 86-02 (CERN, Geneva, 1986), Vol. 1, p. 47;

O. Nicrosini and L. Trentadue, Phys. Lett. B 196 (1987) 551; Z. Phys. C 39 (1988) 479;

F. A. Berends, W. L. van Neerven and G. J. Burgers, Nucl. Phys. B 297 (1988) 429; Erratum-ibid. B 304 (1988) 921.

[19] S. Dittmaier, Nucl. Phys. B 565 (2000) 69 hep-ph/9904440.

[20] M. Roth, dissertation ETH Zürich No. 13363, 1999, hep-ph/0008033.

[21] F. A. Berends, P. H. Daverveldt and R. Kleiss, Nucl. Phys. B 253 (1985) 441;

J. Hilgart, R. Kleiss and F. Le Diberder, Comput. Phys. Commun. 75 (1993) 191.

[22] R. Kleiss and R. Pittau, Comput. Phys. Commun. 83 (1994) 141 hep-ph/9405257.

[23] T. Sjöstrand, L. Lönnblad and S. Mrenna, hep-ph/0108264.

[24] T. Sjöstrand, in Z Physics at LEP1, eds. G. Altarelli, R. Kleiss, and C. Verzegnassi, CERN 89-08 (Geneva, 1989), Vol. 3, p. 143.

[25] B. W. Harris and J. F. Owens, Phys. Rev. D 65 (2002) 094032 hep-ph/0102128].

[26] E. Byckling and K. Kajantie, Particle Kinematics, John Wiley \& Sons, London (1973), p. $158 \mathrm{ff}$.

[27] F. A. Berends, R. Pittau and R. Kleiss, Nucl. Phys. B424 (1994) 308.

[28] S. Dittmaier, Phys. Rev. D59 (1999) 016007.

[29] V. S. Fadin, V. A. Khoze and A. D. Martin, Phys. Lett. B311 (1993) 311;

D. Bardin, W. Beenakker and A. Denner, Phys. Lett. B317 (1993) 213;

V. S. Fadin et al., Phys. Rev. D52 (1995) 1377.

[30] M. Böhm, A. Denner and S. Dittmaier, Nucl. Phys. B376 (1992) 29; E: B391 (1993) 483.

[31] M. Böhm et al., Nucl. Phys. B304 (1988) 463.

[32] J. Fleischer, F. Jegerlehner and M. Zrałek, Z. Phys. C42 (1989) 409.

[33] G. Passarino and M. Veltman, Nucl. Phys. B160 (1979) 151.

[34] D. Yu. Bardin, S. Riemann and T. Riemann, Z. Phys. C32 (1986) 121;

F. Jegerlehner, Z. Phys. C32 (1986) 425. 
[35] A. Denner and T. Sack, Z. Phys. C46 (1990) 653.

[36] G. Gounaris et al., in Physics at LEP2, eds. G. Altarelli, T. Sjöstrand and F. Zwirner (CERN 96-01, Geneva, 1996), Vol. 1, p. 525 hep-ph/9601233.

[37] G. J. Gounaris, J. Layssac and F. M. Renard, Phys. Rev. D 61 (2000) 073013 hep$\mathrm{ph} / 9910395$.

[38] G. J. Gounaris, J. Layssac and F. M. Renard, Phys. Rev. D 62 (2000) 073012 hep$\mathrm{ph} / 0005269$.

[39] P. Sikivie, L. Susskind, M. Voloshin and V. Zakharov, Nucl. Phys. B 173 (1980) 189.

[40] W. J. Stirling and A. Werthenbach, Eur. Phys. J. C 14 (2000) 103 hep-ph/9903315.

[41] G. Bélanger and F. Boudjema, Phys. Lett. B 288 (1992) 201.

[42] O. J. Eboli, M. C. Gonzalez-Garcia and S. F. Novaes, Nucl. Phys. B 411 (1994) 381 hepph/9306306];

G. Abu Leil and W. J. Stirling, J. Phys. G21 (1995) 517 hep-ph/9406317.

[43] W. J. Stirling and A. Werthenbach, Eur. Phys. J. C 12 (2000) 441 hep-ph/9905341.

[44] G. Bélanger, F. Boudjema, Y. Kurihara, D. Perret-Gallix and A. Semenov, Eur. Phys. J. C 13 (2000) 283 hep-ph/9908254.

[45] R. Brun, O. Couet, C. E. Vandoni and P. Zanarini, Comput. Phys. Commun. 57 (1989) 432.

[46] R. Brun and D. Lienart, CERN-Y250. 